\title{
Synthesis of Bicyclic P,S-Heterocycles via Addition of Thioketones to a Phosphorus-Centered Open-Shell Singlet Biradical
}

Henrik Beer, ${ }^{[a]}$ Alexander Linke, ${ }^{[a, b]}$ Jonas Bresien, ${ }^{[a]}$ Grzegorz Mlostoń, ${ }^{[c] *}$ Małgorzata Celeda, ${ }^{[c]}$ Alexander Villinger, and Axel Schulz, ${ }^{[a, b]^{*}}$

[a] A. Linke, H. Beer, Dr. J. Bresien, Dr. A. Villinger, Prof. Dr. A. Schulz,

Institut für Chemie, Universität Rostock, Albert-Einstein-Straße 3a, D-18059 Rostock, Germany

E-mail: axel.schulz@uni-rostock.de

[b] A. Linke, Prof. Dr. A. Schulz

Leibniz-Institut für Katalyse e.V. an der Universität Rostock, Albert-Einstein-Straße 29a, D-18059 Rostock, Germany.

[c] Prof. Dr. Grzegorz Mlostoń, Małgorzata Celeda,

Department of Organic and Applied Chemistry, University of Łódź, Tamka 12, PL-91-403 Łódź, Poland

E-mail: grzegorz.mloston@chemia.uni.lodz.pl

\section{SUPPORTING INFORMATION}

This file includes:

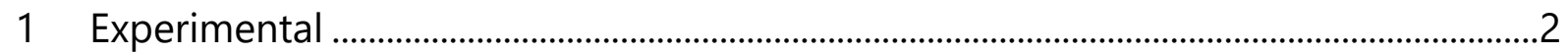

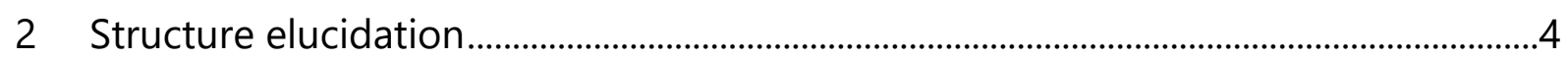

3 Syntheses of compounds ......................................................................................................

4 Additional spectroscopic details.......................................................................................23

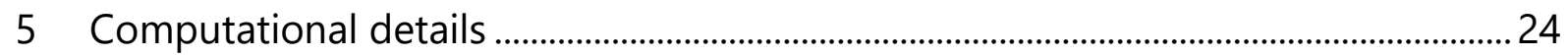

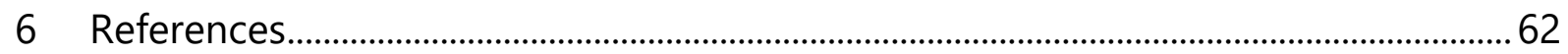




\section{Experimental}

General Information. If not stated otherwise, all manipulations were carried out under oxygen- and moisture-free conditions in an inert argon atmosphere using standard Schlenk or drybox techniques. All glassware was heated three times in vacuo using a heat gun and cooled under argon atmosphere. Solvents were transferred using syringes, which were purged three times with argon prior to use. Solvents and reactants were either obtained from commercial sources or synthesized as detailed in Table S1.

Table S1: Origin and purification of solvents and reactants.

\begin{tabular}{|c|c|c|}
\hline Substance & Origin & Purification \\
\hline THF & local trade & $\begin{array}{l}\text { dried over } \mathrm{Na} / \text { benzophenone } \\
\text { freshly distilled prior to use }\end{array}$ \\
\hline$n$-hexane & local trade & $\begin{array}{l}\text { dried over } \mathrm{Na} \text { /benzophenone/tetraglyme } \\
\text { freshly distilled prior to use }\end{array}$ \\
\hline $\mathrm{C}_{6} \mathrm{H}_{6}$ & local trade & $\begin{array}{l}\text { dried over } \mathrm{Na} / \text { benzophenone/tetraglyme } \\
\text { freshly distilled prior to use }\end{array}$ \\
\hline Toluene & local trade & $\begin{array}{l}\text { dried over } \mathrm{Na} / \text { benzophenone/tetraglyme } \\
\text { freshly distilled prior to use }\end{array}$ \\
\hline $\mathrm{C}_{6} \mathrm{D}_{6}$ & euriso-top & $\begin{array}{l}\text { dried over } \mathrm{Na} \\
\text { freshly distilled prior to use }\end{array}$ \\
\hline Tol- $d_{8}$ & euriso-top & $\begin{array}{l}\text { dried over } \mathrm{Na} \\
\text { freshly distilled prior to use }\end{array}$ \\
\hline THF- $d_{8}$ & euriso-top & $\begin{array}{l}\text { dried over } \mathrm{Na} \\
\text { distilled and stored over molecular sieves ( } 4 \AA \text { ) }\end{array}$ \\
\hline$[\mathrm{P}(\mu-\mathrm{NTer})]_{2}$ & synthesized $^{[1]}$ & re-crystallized as described in the literature \\
\hline 9H-xanthene-9-on & $\begin{array}{l}\text { commercially available } \\
\text { reagent }\end{array}$ & sublimated at $110^{\circ} \mathrm{C}$ (oil bath, $1 \times 10^{-3} \mathrm{mbar}$ ) \\
\hline $9 H$-xanthene-9-thione & synthesized ${ }^{[2]}$ & column chromatography \\
\hline $9 H$-thioxanthene-9-thione & Synthesized ${ }^{[2]}$ & column chromatography \\
\hline Fc- $\beta$-naph-thioketone & Synthesized ${ }^{[3]}$ & column chromatography \\
\hline
\end{tabular}


NMR spectra were recorded on Bruker spectrometers (AVANCE 250, AVANCE 300 or AVANCE 500) and were referenced internally to the deuterated solvent $\left({ }^{13} \mathrm{C}_{0} \mathrm{C}_{6} \mathrm{D}_{6}\right.$ $\delta_{\text {ref }}=128.4$ ppm, Toluene- $d_{8} \delta_{\text {Ref, } 1}=20.4$ ppm, $\delta_{\text {Ref, } 2}=125.5$ ppm, $\delta_{\text {Ref, } 3}=128.3$ ppm, $\delta_{\text {Ref, } 4}=129.2$ ppm, $\delta_{\text {Ref, } 5}=137.9$ ppm, THF- $d_{8} \delta_{\text {ref, } 1}=25.4$ ppm, $\delta_{\text {ref, } 2}=67.6$ ppm), to protic impurities in the deuterated solvent $\left({ }^{1} \mathrm{H}: \mathrm{C}_{6} \mathrm{HD}_{5} \delta_{\text {ref }}=7.16 \mathrm{ppm}\right.$, Toluene- $\mathrm{d}_{8}$ $\delta_{\text {Ref, } 1}=2.09 \mathrm{ppm}, \delta_{\text {Ref, } 2}=6.98 \mathrm{ppm}, \delta_{\text {Ref,3 }}=7.00 \mathrm{ppm}, \delta_{\text {Ref, } 4}=7.09 \mathrm{ppm}$, THF $-d_{7}$ $\left.\delta_{\text {ref, } 1}=1.73 \mathrm{ppm}, \delta_{\text {ref, } 2}=3.58 \mathrm{ppm}\right)$ or externally $\left({ }^{31} \mathrm{P}: 85 \% \mathrm{H}_{3} \mathrm{PO}_{4} \delta_{\text {ref }}=0 \mathrm{ppm}\right)$. All measurements were carried out at ambient temperature unless denoted otherwise. NMR signals were assigned using experimental data (e.g. chemical shifts, coupling constants, integrals where applicable) in conjunction with computed NMR data (GIAO method, cf. Computational details, p. S23). The signs of ${ }^{n} J\left({ }^{31} \mathrm{P},{ }^{31} \mathrm{P}\right)$ coupling constants were derived from calculated values.

IR spectra of crystalline samples were recorded on a Bruker Alpha II FT-IR spectrometer equipped with an ATR unit at ambient temperature under argon atmosphere. Relative intensities are reported according to the following intervals: very weak ( $\mathrm{vw}, 0-10 \%)$, weak (w, 10-30\%), medium ( $\mathrm{m}, 30-60 \%)$, strong (s, 60-90\%), very strong (vs, 90-100\%).

Raman spectra of crystalline samples were recorded using a LabRAM HR 800 Horiba Jobin YVON Raman spectrometer equipped with an Olympus BX41 microscope with variable lenses. The samples were excited by a red laser $(633 \mathrm{~nm}, 17 \mathrm{~mW}$, air-cooled HeNe laser). All measurements were carried out at ambient temperature unless stated otherwise.

Elemental analyses were obtained using an Elementar vario Micro cube CHNS analyser.

Melting points (uncorrected) were determined using a Stanford Research Systems EZ Melt at a heating rate of $20^{\circ} \mathrm{C} / \mathrm{min}$. Clearing points are reported.

Mass spectra were recorded on a Thermo Electron MAT 95-XP sector field mass spectrometer using crystalline samples. 


\section{Structure elucidation}

X-ray Structure Determination: X-ray quality crystals were selected in Fomblin YR1800 perfluoroether (Alfa Aesar) at ambient temperature. The samples were cooled to 123(2) K during measurement. The data were collected on a Bruker D8 Quest diffractometer or a Bruker Kappa Apex II diffractometer using Mo $\mathrm{K}_{\alpha}$ radiation $(\lambda=0.71073 \AA)$. The structures were solved by iterative methods (SHELXT) ${ }^{[S 4]}$ and refined by full matrix least squares procedures $(\mathrm{SHELXL}){ }^{[\mathrm{S} 5]}$ Semi-empirical absorption corrections were applied (SADABS). ${ }^{[S 6]}$ All non-hydrogen atoms were refined anisotropically, hydrogen atoms were included in the refinement at calculated positions using a riding model.

\section{$2.1 \quad\left([\mathrm{P}(\mu-\mathrm{NTer})]_{2} \cdot 9 H\right.$-thioxanthene-9-thione) (3SS)}

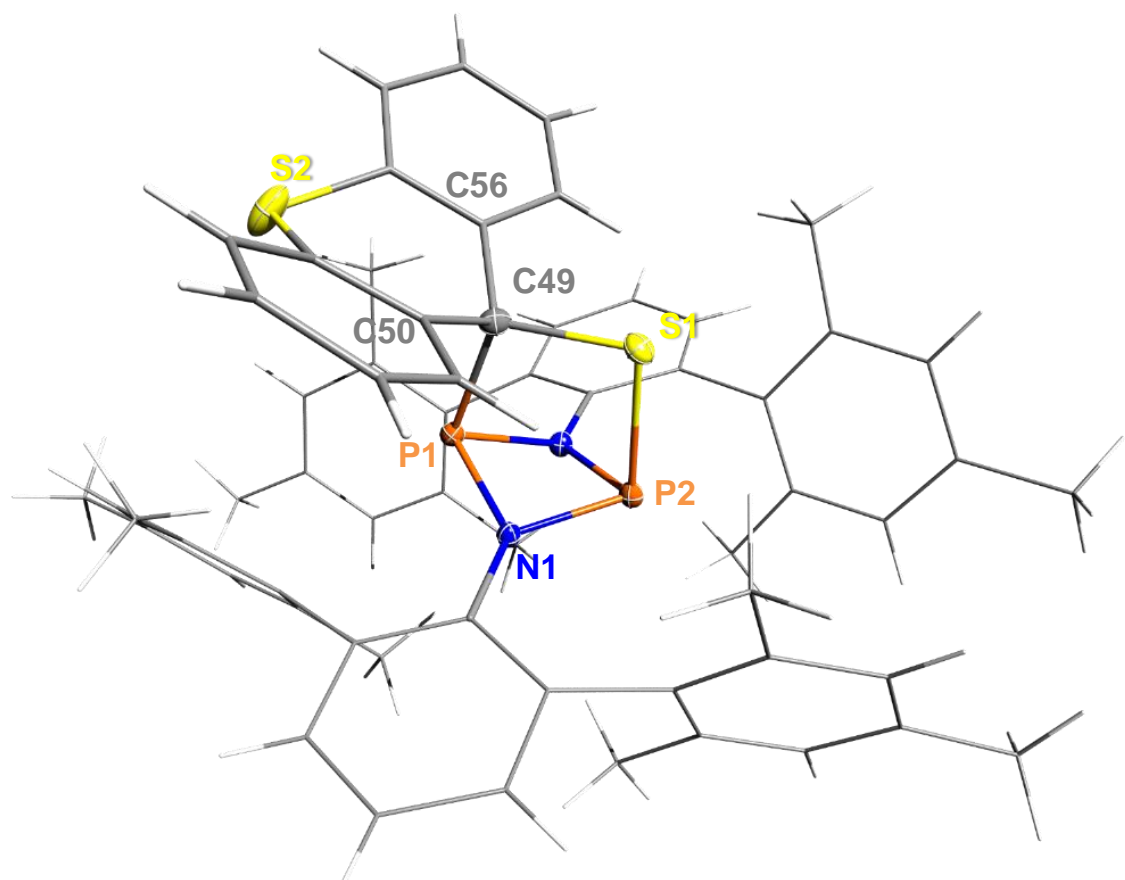




\section{$2.2\left([\mathrm{P}(\mu-\mathrm{NTer})]_{2} \cdot 9 H\right.$-xanthene-9-thione) (3OS)}

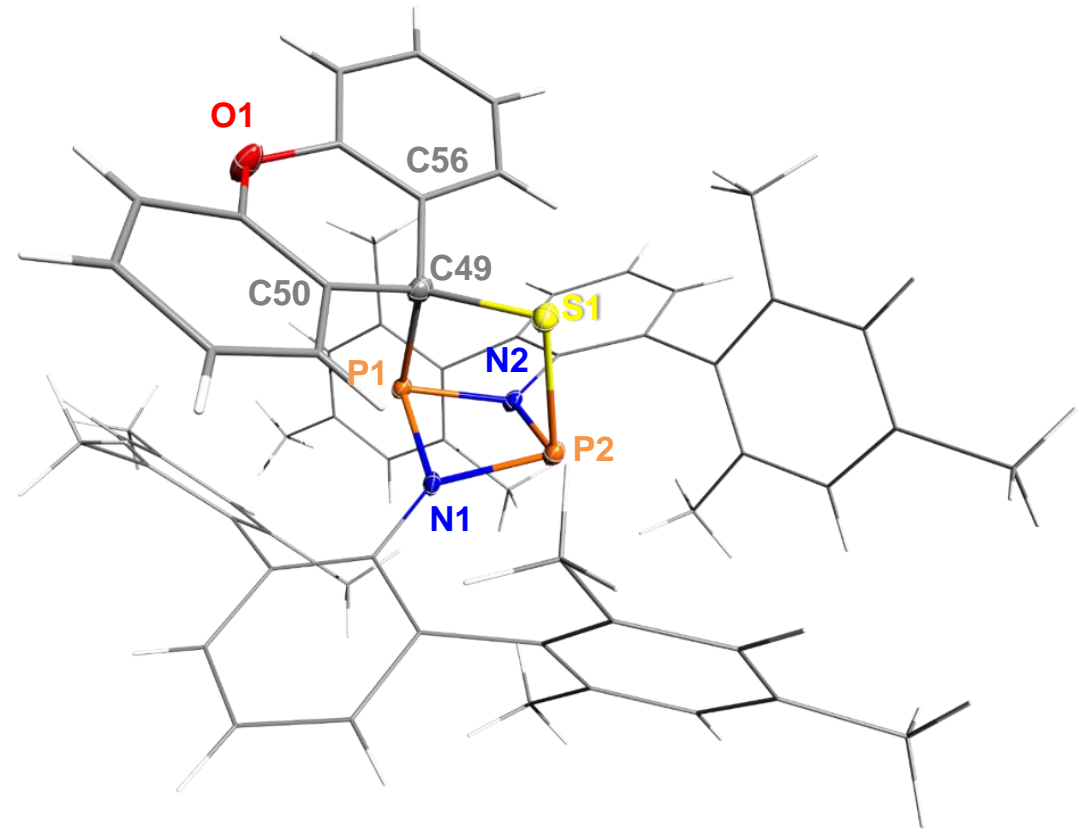

$2.3\left([\mathrm{P}(\mu-\mathrm{NTer})]_{2} \cdot \mathrm{Fc}-\beta\right.$-naphtyl-thioketone $)(3 \mathrm{Fc})$

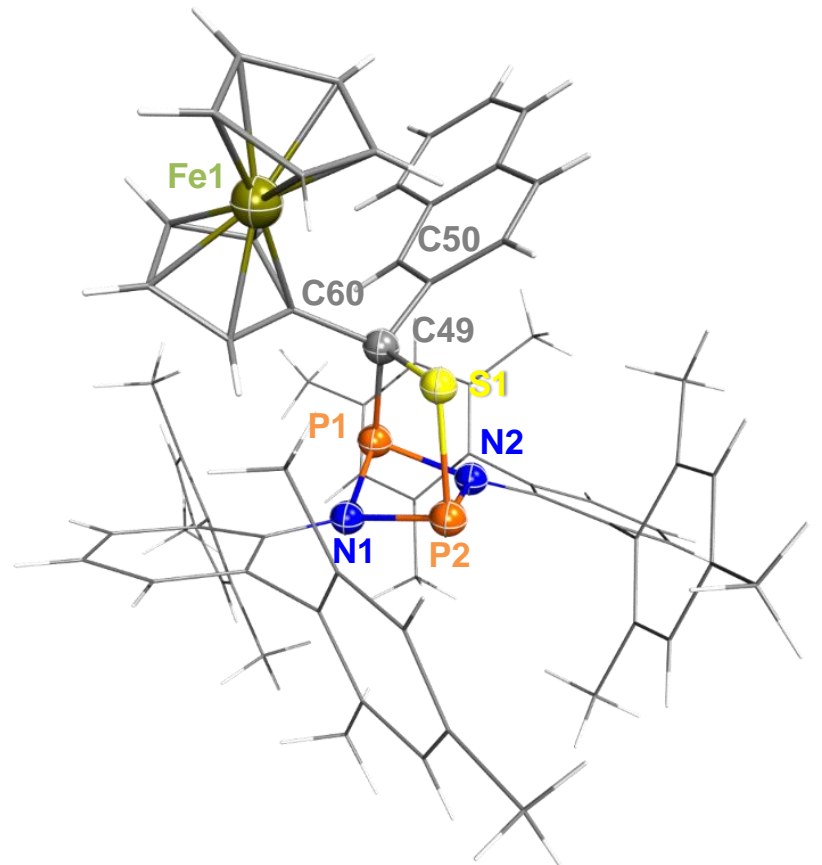

Only structural evidence due to low data quality. Heteroatoms are represented as "ball and stick". 
Table S2: Crystallographic details.

\begin{tabular}{|c|c|c|c|}
\hline Compound & $\begin{array}{l}{[\mathrm{P}(\mu \text {-NTer })]_{2} \cdot 9 \mathrm{H}-} \\
\text { xanthene-9-thione }\end{array}$ & $\begin{array}{l}{[\mathrm{P}(\mu-\mathrm{NTer})]_{2} \cdot 9 \mathrm{H}-} \\
\text { thioxanthene-9-thione }\end{array}$ & $\begin{array}{l}{[\mathrm{P}(\mu-\mathrm{NTer})]_{2} \cdot \mathrm{Fc}-\beta-} \\
\text { naphtyl-thioketone }{ }^{[\mathrm{a}]}\end{array}$ \\
\hline Chem. Formula & $\mathrm{C}_{61} \mathrm{H}_{58} \mathrm{~N}_{2} \mathrm{P}_{2} \mathrm{OS} \cdot \mathrm{C}_{7} \mathrm{H}_{8}$ & $\mathrm{C}_{61} \mathrm{H}_{58} \mathrm{~N}_{2} \mathrm{P}_{2} \mathrm{~S}_{2} \cdot \mathrm{C}_{6} \mathrm{H}_{6}$ & $\mathrm{C}_{69} \mathrm{H}_{66} \mathrm{~N}_{2} \mathrm{P}_{2} \mathrm{SFe}$ \\
\hline Formula weight $[\mathrm{g} / \mathrm{mol}]$ & 1021.22 & 1023.26 & 1073.15 \\
\hline Colour & colourless & yellow & yellow \\
\hline Crystal system & monoclinic & monoclinic & triclinic \\
\hline Space group & $P 2_{1} / n$ & $P 2_{1} / n$ & $P \overline{1}$ \\
\hline$a[\AA]$ & 12.799(3) & $12.8246(4)$ & $12.614(5)$ \\
\hline$b[\AA]$ & $11.644(3)$ & $11.7095(4)$ & $12.782(5)$ \\
\hline$c[\AA]$ & $36.501(9)$ & $36.4470(14)$ & $18.811(7)$ \\
\hline$\alpha\left[^{\circ}\right]$ & 90 & 90 & $70.917(12)$ \\
\hline$\beta\left[^{\circ}\right]$ & $92.765(9)$ & $92.8710(10)$ & $89.639(12)$ \\
\hline$\gamma\left[^{\circ}\right]$ & 90 & 90 & $74.785(13)$ \\
\hline$V\left[\AA^{3}\right]$ & $5433(2)$ & $5466.4(3)$ & $2755.5(19)$ \\
\hline Z & 4 & 4 & 2 \\
\hline$\rho_{\text {calcd. }}\left[\mathrm{g} / \mathrm{cm}^{3}\right]$ & 1.248 & 1.243 & 1.293 \\
\hline$\mu\left[\mathrm{mm}^{-1}\right]$ & 0.165 & 0.200 & 0.42 \\
\hline$T[\mathrm{~K}]$ & $123(2)$ & $123(2)$ & $123(2)$ \\
\hline Measured reflections & 51331 & 118786 & 6347 \\
\hline Independent reflections & 14420 & 17413 & 6347 \\
\hline Reflections with $I>2 \sigma(I)$ & 7265 & 12643 & 2843 \\
\hline$R_{\text {int }}$ & 0.161 & 0.065 & - \\
\hline$F(000)$ & 2168 & 2168 & 1132 \\
\hline$R_{1}\left(R\left[F^{2}>2 \sigma\left(F^{2}\right)\right]\right)$ & 0.074 & 0.047 & 0.102 \\
\hline $\mathrm{w} R_{2}\left(F^{2}\right)$ & 0.155 & 0.113 & 0.2581 \\
\hline GooF & 0.992 & 1.040 & 0.923 \\
\hline No. of Parameters & 733 & 713 & 354 \\
\hline CCDC \# & 2114484 & 2114485 & - \\
\hline
\end{tabular}

[a] As the crystals were very small, the data quality is rather poor, and the structure solution only serves as proof of connectivity. 


\section{Syntheses of compounds}

\section{1 $\left[\mathrm{P}(\mu \text {-NTer) }]_{2} \cdot 9 H\right.$-xanthene-9-thione (3OS)}<smiles>S=c1c2ccccc2oc2cc[cH+]ccc12</smiles>



9H-xanthene-9-thione (59 mg, $0.28 \mathrm{mmol})$ and $[\mathrm{P}(\mu-\mathrm{NTer})]_{2}(200 \mathrm{mg}, 0.28 \mathrm{mmol})$ were combined and dissolved in benzene $(15 \mathrm{~mL})$. An immediate colour change from red to greenish-yellow was observed. After stirring for one hour, all volatile components were removed in vacuo $\left(1 \times 10^{-3} \mathrm{mbar}\right)$ and the yellow residue was dried in vacuo $\left(1 \times 10^{-3} \mathrm{mbar}\right)$ for $30 \mathrm{~min}$ at $40^{\circ} \mathrm{C}$ (water bath). The product was crystallized from a minimal amount of fresh benzene at ambient temperature. The supernatant was removed by syringe and the crystals were dried in vacuo $\left(1 \times 10^{-3} \mathrm{mbar}\right)$ for 30 minutes at $40{ }^{\circ} \mathrm{C}$ (water bath). Yield: $140 \mathrm{mg}(0.660 \mathrm{mmol}, 54 \%)$.

After recrystallization from a saturated toluene solution at a slightly elevated temperature, crystals suitable for X-ray diffraction were obtained at ambient temperature.

$\mathbf{C}_{61} \mathbf{H}_{\mathbf{5}} \mathbf{N}_{\mathbf{2}} \mathbf{P}_{\mathbf{2}} \mathbf{O S}$ (929.16 g/mol) Mp. $107^{\circ} \mathrm{C}$ (decomp.). CHN calc. (found) in \%: C 78.85 (78.58), H 6.29 (6.33), N 3.01 (2.68), S 3.45 (2.83). ${ }^{1} \mathbf{H}$ NMR $\left(25^{\circ} \mathrm{C}, \mathrm{C}_{6} \mathrm{D}_{6}, 500.1 \mathrm{MHz}\right)$ : $\delta=1.06\left(\mathrm{~s}, 6 \mathrm{H}, o-\mathrm{CH}_{3}\right), 2.28\left(\mathrm{~s}, 6 \mathrm{H}, o-\mathrm{CH}_{3}\right), 2.33\left(\mathrm{~s}, 6 \mathrm{H}, o-\mathrm{CH}_{3}\right), 2.35\left(\mathrm{~s}, 12 \mathrm{H}, p-\mathrm{CH}_{3}\right)$, $2.56\left(\mathrm{~s}, 6 \mathrm{H}, \mathrm{o}-\mathrm{CH}_{3}\right), 6.44(\mathrm{~m}, 4 \mathrm{H}$, arom. $\mathrm{CH}), 6.66(\mathrm{~m}, 13 \mathrm{H}$, arom. $\mathrm{CH}), 6.84(\mathrm{~s}, 2 \mathrm{H}$, arom. $\mathrm{CH}), 6.91(\mathrm{~d}, 2 \mathrm{H}$, arom. $\mathrm{CH}), 7.41\left(\mathrm{~m}, 2 \mathrm{H}\right.$, arom. $p-\mathrm{CH} C H^{13} \mathrm{C}\left\{{ }^{1} \mathrm{H}\right\}$ NMR $\left(25^{\circ} \mathrm{C}, \mathrm{C}_{6} \mathrm{D}_{6}\right.$, 125.8 MHz): $\delta=19.9\left(\mathrm{~s}, \mathrm{CH}_{3}\right), 21.8\left(\mathrm{~s}, \mathrm{CH}_{3}\right), 22.0\left(\mathrm{~s}, \mathrm{CH}_{3}\right), 22.3\left(\mathrm{~s}, \mathrm{CH}_{3}\right), 22.6\left(\mathrm{~s}, \mathrm{CH}_{3}\right)$, 117.3 (s, arom. C), 121.2 (s, arom. C), 123.0 (s, arom. C), 125.8 (s, arom. C), 126.0 (s, arom. C), 128.9 (s, arom. C), 129.0 (s, arom. C), 129.2 (s, arom. C), 129.2 (s, arom. C), 129.7 (s, arom. C), 129.8 (s, arom. C), 130.3 (s, arom. C), 131.5 (s, arom. C), 132.9 (s, quart. C), 
133.1 (s, quart. C), 133.5 (s, quart. C), 136.8 (s, quart. C), 137.9 (s, quart. C), 138.0 (s, quart. C), 140.2 (s, quart. C). ${ }^{31} \mathbf{P}\left\{{ }^{1} \mathbf{H}\right\} \mathbf{N M R}\left(25^{\circ} \mathrm{C}_{1} \mathrm{C}_{6} \mathrm{D}_{6}, 101.3 \mathrm{MHz}\right): \delta=215.0(\mathrm{~d}, 1 \mathrm{P}$, $\left.{ }^{2} J\left({ }^{31} \mathrm{P}_{1}^{31} \mathrm{P}\right)=33 \mathrm{~Hz}\right), \quad 251.5\left(\mathrm{~d}, 1 \mathrm{P}, \quad{ }^{2} J\left({ }^{31} \mathrm{P},{ }^{31} \mathrm{P}\right)=33 \mathrm{~Hz}\right) . \quad{ }^{15} \mathrm{~N} \mathrm{HMBC} \quad\left(25{ }^{\circ} \mathrm{C}, \mathrm{C}_{6} \mathrm{D}_{6}\right.$, 50.7 MHz): $\delta=2113.1 .{ }^{14} \mathbf{N}$ NMR $\left(25{ }^{\circ} \mathrm{C}_{1} \mathrm{C}_{6} \mathrm{D}_{6}, 18.1 \mathrm{MHz}\right)$ : No signals observed. IR (ATR, 32 scans, $\left.\mathrm{cm}^{-1}\right): \tilde{v}=2949(\mathrm{w}), 2916(\mathrm{w}), 2854(\mathrm{w}), 2726(\mathrm{w}), 1609(\mathrm{w}), 1597(\mathrm{vw}), 1591$ $(w), 1576(w), 1568(w), 1471(m), 1436(m), 1401(s), 1374(m), 1292(m), 1278(m), 1232$ (s), $1220(s), 1170(w), 1152(w), 1119(w), 1090(m), 1032(w), 1014(w), 1004(w), 946$ (w), $900(\mathrm{~m}), 894(\mathrm{~m}), 845(\mathrm{vs}), 795(\mathrm{~m}), 777(\mathrm{~m}), 748(\mathrm{~s}), 734(\mathrm{~s}), 692(\mathrm{~s}), 645(\mathrm{w}), 626$ (w), $577(w), 560(w), 546(w), 528(w), 513(w), 495(w), 464(m), 443$ (m), 437 (m). Raman (633 nm, 10 s, 15 scans, cm $\left.{ }^{-1}\right): \tilde{v}=3069$ (1), 3053 (1), 3043 (1), 3013 (1), 2950 (1), 2922 (2), 2856 (1), 2725 (1), 1611 (2), 1591 (1), 1577 (1), 1485 (1), 1455 (1), 1436 (1), 1415 (1), 1402 (1), 1384 (1), 1378 (1), 1372 (1), 1301 (3), 1276 (1), 1242 (1), 1232 (1), 1212 (3), 1194 (1), 1170 (2), 1151 (3), 1118 (1), 1098 (1), 1068 (1), 1035 (4), 1001 (3), 945 (1), 893 (1), 856 (1), 847 (1), 806 (1), 794 (1), 782 (1), 776 (1), 748 (1), 739 (1), 704 (1), 692 (1), 682 (1), 650 (2), 627 (1), 590 (1), 589 (1), 586 (1), 577 (5), 560 (3), 553 (1), 533 (1), 523 (1), 512 (1), 488 (1), 472 (1), 463 (1), 436 (1), 426 (1), 403 (1), 382 (1), 375 (1), 368 (1), 336 (1), 326 (1), 290 (1), 272 (1), 258 (1), 243 (1), 228 (1), 214 (1), 199 (2), 168 (1), 149 (2), 134 (2), 107 (4), 90 (9), 77 (10). MS (Cl pos., iso-butane) m/z (\%): 69, 103, 213 $\left[\mathrm{C}_{13} \mathrm{H}_{8} \mathrm{OS}\right]^{+}, 330\left[\text { Ter- } \mathrm{NH}_{3}\right]^{+}, 386\left[\text { Ter- } \mathrm{NH}_{3} \text { iso-butane }\right]^{+}, 508,687,716\left[[\mathrm{P}(\mu-\mathrm{NTer})]_{2}\right]^{+}$, $910\left[\mathrm{C}_{55} \mathrm{H}_{54} \mathrm{~N}_{2} \mathrm{P}_{2} \mathrm{SO}+\text { iso-butane }\right]^{+}$. 
Figure S1: NMR, IR and Raman spectra of $[\mathrm{P}(\mu-\mathrm{NTer})]_{2} \cdot 9 \mathrm{H}$-xanthene-9-thione (solvent signals indicated by asterisks).

\section{${ }^{1} \mathrm{H}$ NMR spectrum}
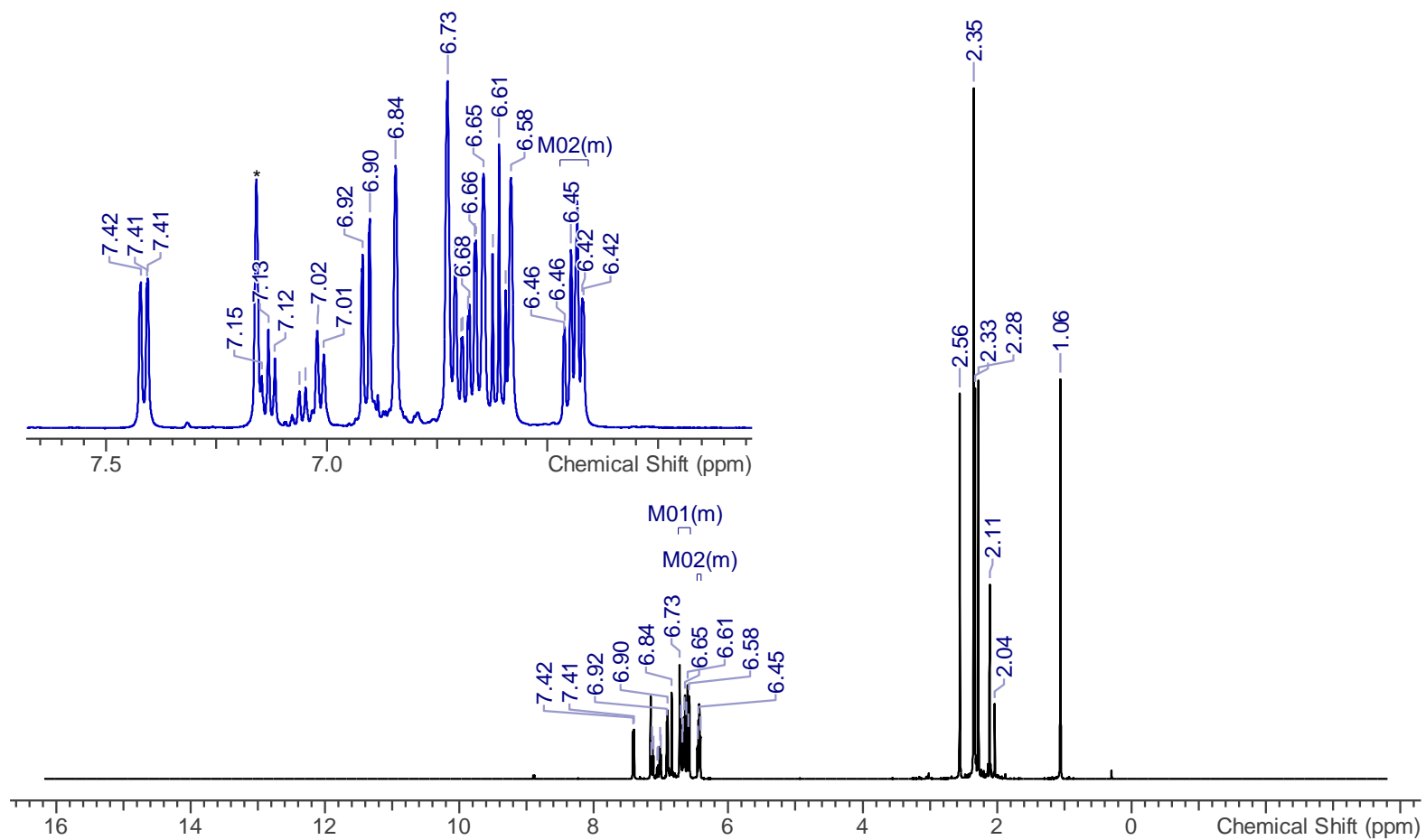

\section{${ }^{13} \mathrm{C}\left\{{ }^{1} \mathrm{H}\right\}$ NMR spectrum}

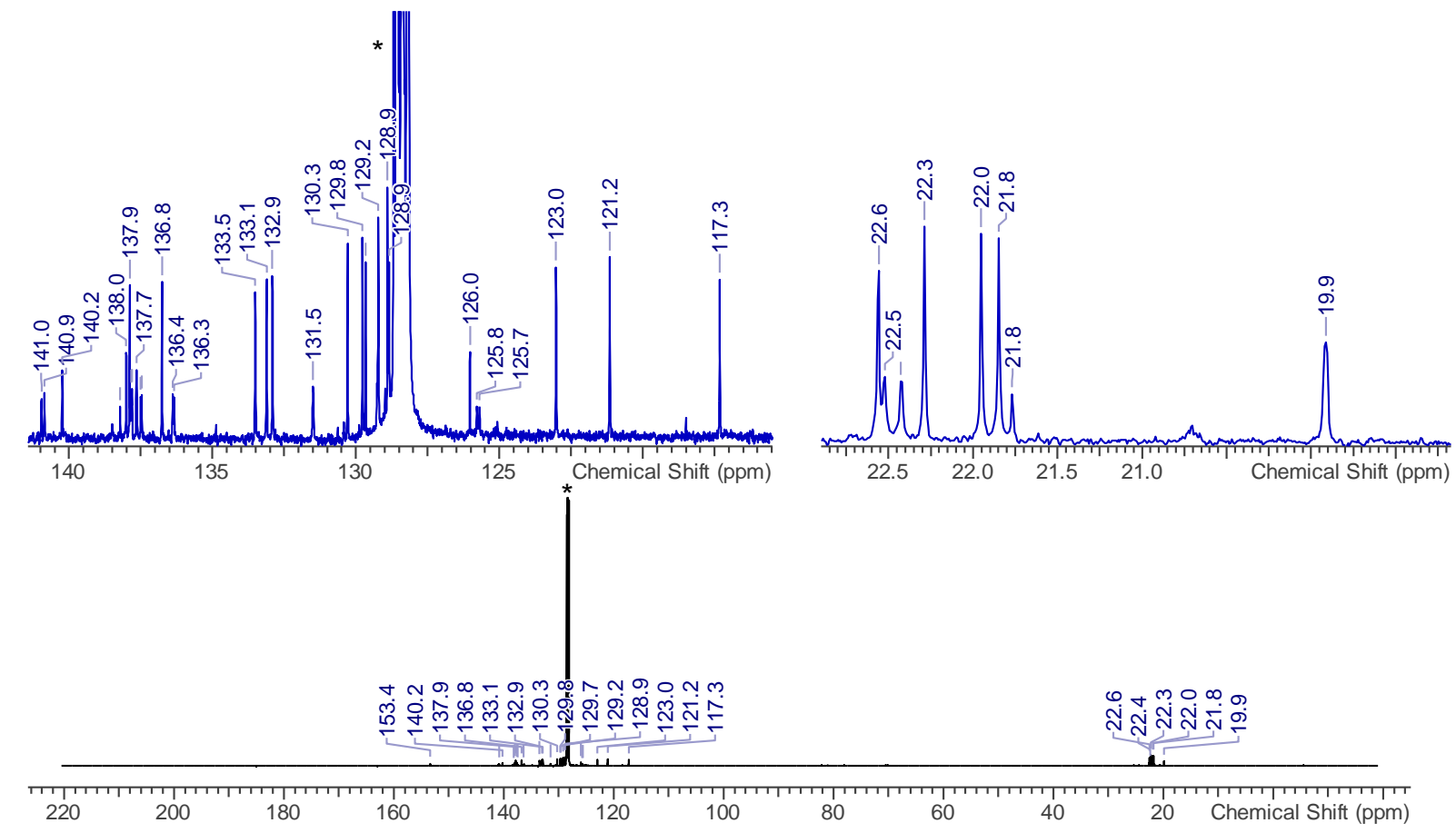


Figure S1: NMR, IR and Raman spectra of $[\mathrm{P}(\mu$-NTer $)] 2 \cdot 9 \mathrm{H}$-xanthene-9-thione (solvent signals indicated by asterisks).continued.
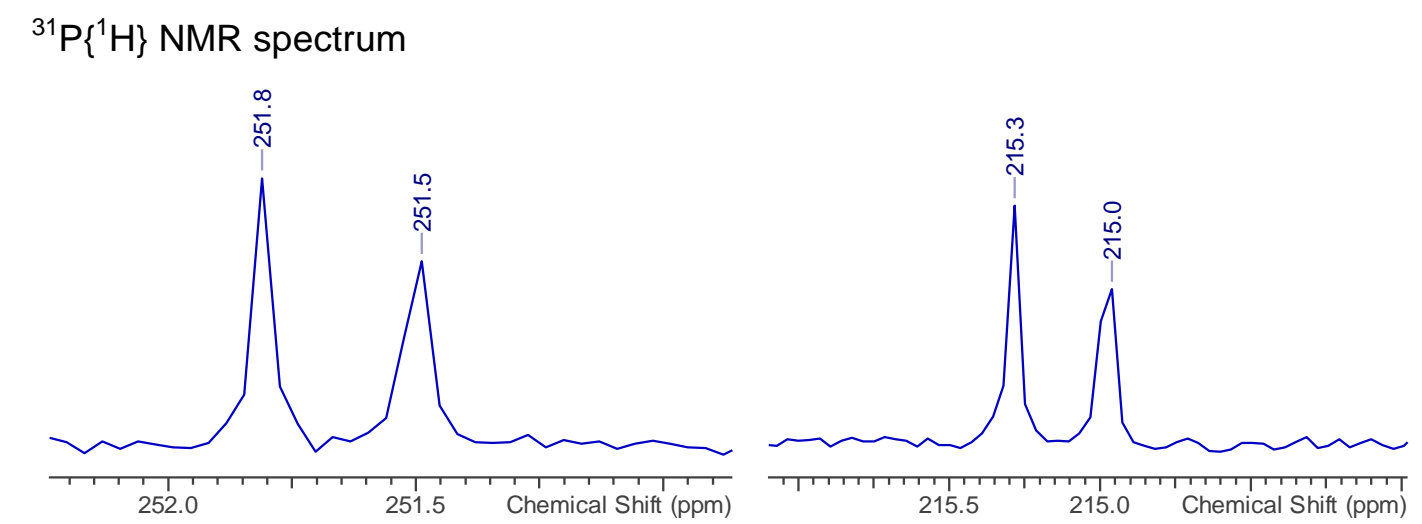

$\stackrel{\infty}{\infty} \underset{\sim}{\infty} \frac{m}{N}$

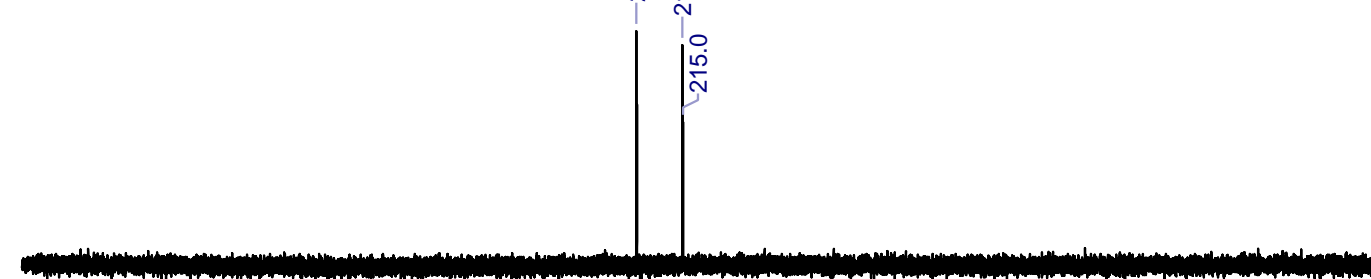

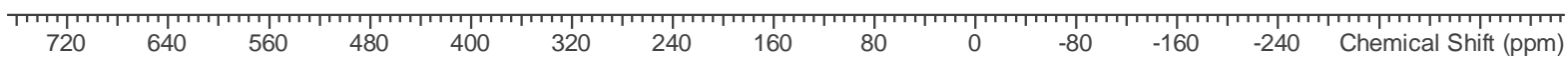
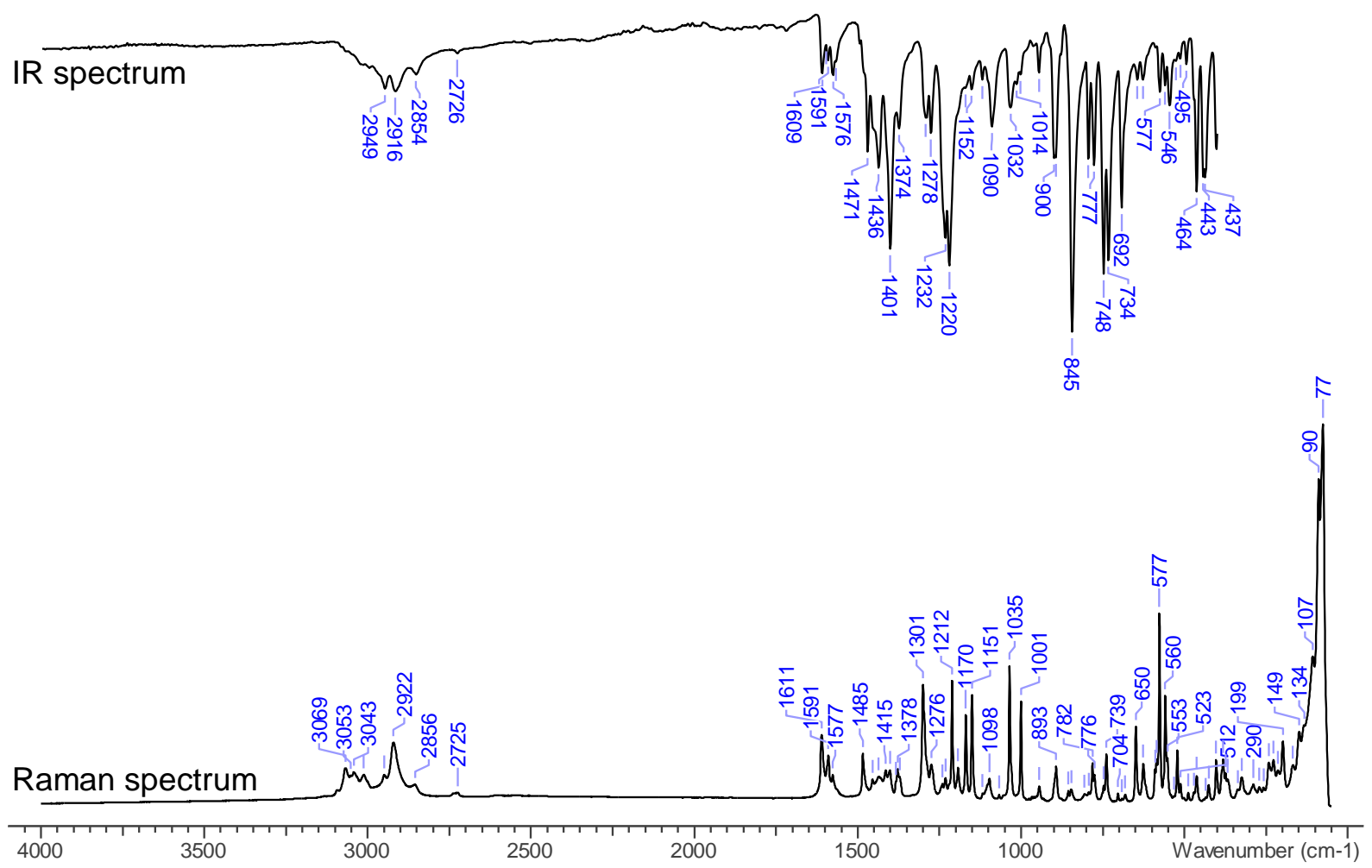


\section{$3.2\left[\mathrm{P}(\mu \text {-NTer) }]_{2} \cdot 9 \mathrm{H}\right.$-thioxanthene-9-thione (3SS)}
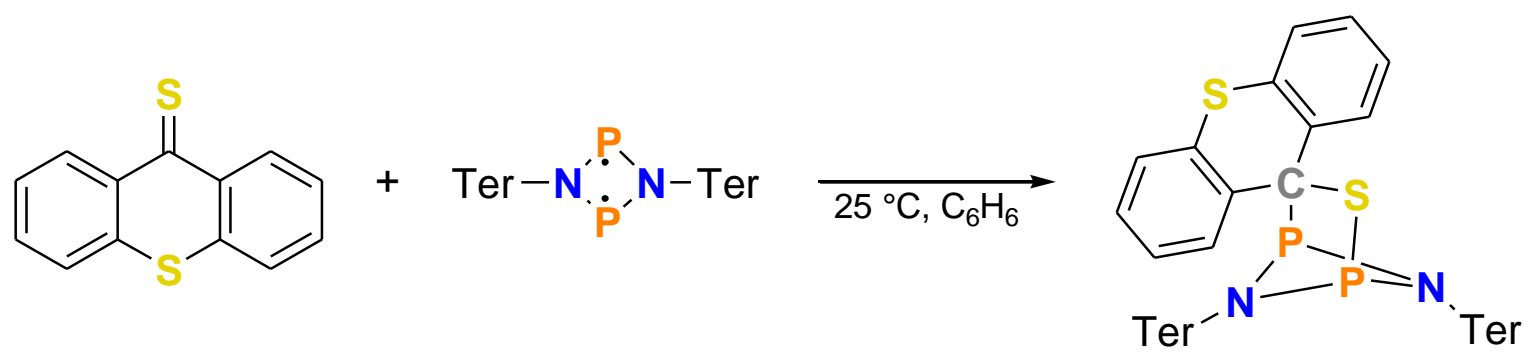

$9 \mathrm{H}$-thioxanthene-9-thione $(64 \mathrm{mg}, 0.28 \mathrm{mmol})$ and $[\mathrm{P}(\mu-\mathrm{NTer})]_{2}(200 \mathrm{mg}, 0.28 \mathrm{mmol})$ were combined and dissolved in benzene $(15 \mathrm{~mL})$. An immediate colour change from red to green was observed. After stirring for one hour, all volatile components were removed in vacuo $\left(1 \times 10^{-3} \mathrm{mbar}\right)$ and the greenish residue was dried in vacuo $\left(1 \times 10^{-3} \mathrm{mbar}\right)$ for $30 \mathrm{~min}$ at $40^{\circ} \mathrm{C}$ (water bath). The product was crystallized from a minimal amount of fresh benzene at ambient temperature. The supernatant was removed by syringe and the crystals were dried in vacuo $\left(1 \times 10^{-3} \mathrm{mbar}\right)$ for 30 minutes at $40^{\circ} \mathrm{C}$ (water bath). Yield: $165 \mathrm{mg}(0.170 \mathrm{mmol}, 63 \%)$.

After recrystallization from a saturated benzene solution at a slightly elevated temperature, crystals suitable for X-ray diffraction were obtained at ambient temperature.

$\mathbf{C}_{61} \mathbf{H}_{58} \mathbf{N}_{\mathbf{2}} \mathbf{P}_{2} \mathbf{S}_{2}$ (945.22 g/mol). Mp. $107^{\circ} \mathrm{C}$ (decomp.). $\mathbf{C H N}$ calc. (found) in \%: C 77.51 (77.73), H 6.18 (6.09), N 2.96 (2.65), S 6.78 (5.81). ${ }^{1} \mathrm{H}$ NMR $\left(25^{\circ} \mathrm{C}_{1} \mathrm{C}_{6} \mathrm{D}_{6}, 300.1 \mathrm{MHz}\right)$ : $\delta=1.02\left(\mathrm{~s}, 6 \mathrm{H}, \mathrm{CH}_{3}\right), 2.30\left(\mathrm{~s}, 6 \mathrm{H}, \mathrm{CH}_{3}\right), 2.31\left(\mathrm{~s}, 6 \mathrm{H}, \mathrm{CH}_{3}\right), 2.39\left(\mathrm{~s}, 6 \mathrm{H}, \mathrm{CH}_{3}\right), 2.40(\mathrm{~s}, 6 \mathrm{H}$, $\left.\mathrm{CH}_{3}\right), 2.51\left(\mathrm{~s}, 6 \mathrm{H}, \mathrm{CH}_{3}\right), 6.39(\mathrm{~m}, 2 \mathrm{H}$, arom. $\mathrm{CH}), 6.62(\mathrm{~m}, 12 \mathrm{H}$, arom. $\mathrm{CH}), 6.83(\mathrm{~s}, 2 \mathrm{H}$, arom. $\mathrm{CH}), 6.90(\mathrm{~s}, 2 \mathrm{H}$, arom. $\mathrm{CH}), 7.23(\mathrm{~m}, 2 \mathrm{H}$, arom. $\mathrm{CH}), 8.03(\mathrm{~m}, 2 \mathrm{H}$, arom. $\mathrm{CH})$. ${ }^{13} \mathrm{C}\left\{{ }^{1} \mathrm{H}\right\}$ NMR $\left(25^{\circ} \mathrm{C}, \mathrm{C}_{6} \mathrm{D}_{6}, 75.5 \mathrm{MHz}\right): \delta=21.5\left(\mathrm{~s}, \mathrm{CH}_{3}\right), 21.9\left(\mathrm{~s}, \mathrm{CH}_{3}\right), 22.3\left(\mathrm{~s}, \mathrm{CH}_{3}\right), 22.6$ $\left(\mathrm{s}, \mathrm{CH}_{3}\right), 22.7\left(\mathrm{~s}, \mathrm{CH}_{3}\right), 22.8\left(\mathrm{~s}, \mathrm{CH}_{3}\right), 121.1$ (s, arom. C), 125.8 (s, arom. C), 127.6 (s, arom. C), 128.9 (s, arom. C), 129.0 (s, arom. C), 129.3 (s, arom. C), 129.4 (s, arom. C), 129.8 (s, arom. C), 130.0 (s, arom. C), 131.3 (s, arom. C), 132.7 (s, arom. C), 132.8 (s, arom. C), 133.2 (s, arom. C), 134.5 (s, arom. C), 136.8 (s, arom. C), 136.9 (s, arom. C), 137.7 (s, arom. C), 137.9 (s, arom. C), 138.0 (s, arom. C), 139.4 (s, arom. C), 139.5 (s, arom. C), 139.7 (s, 
arom. C), 140.6 (s, arom. C), 140.8 (s, arom. C). ${ }^{31} \mathbf{P}\left\{{ }^{1} \mathrm{H}\right\}$ NMR $\left(25^{\circ} \mathrm{C}_{1} \mathrm{C}_{6} \mathrm{D}_{6}, 121.5 \mathrm{MHz}\right)$ : $\delta=213.5\left(\mathrm{~d}, 1 \mathrm{P},{ }^{2} J\left({ }^{31} \mathrm{P},{ }^{31} \mathrm{P}\right)=31 \mathrm{~Hz}\right), 249.4\left(\mathrm{~d}, 1 \mathrm{P},{ }^{2} J\left({ }^{31} \mathrm{P},{ }^{31} \mathrm{P}\right)=31 \mathrm{~Hz}\right) .{ }^{15} \mathbf{N} \mathbf{~ H M B C}$ $\left(25^{\circ} \mathrm{C}, \mathrm{C}_{6} \mathrm{D}_{6}, 50.7 \mathrm{MHz}\right): \delta=173.1 .{ }^{14} \mathrm{~N}$ NMR $\left(25^{\circ} \mathrm{C}, \mathrm{C}_{6} \mathrm{D}_{6}, 18.1 \mathrm{MHz}\right)$ : No signals observed. IR (ATR, 32 scans, cm ${ }^{-1}$ ): $\tilde{v}=3050$ (w), 2996 (w), 2947 (w), 2914 (w), 2852 (w), $2726(w), 2166(v w), 1723(v w), 1611(w), 1576(w), 1494(w), 1480(w), 1449(m), 1438$ $(m), 1399(s), 1374(m), 1288(w), 1267(w), 1230(s), 1220(s), 1160(w), 1121(w), 1090$ (m), $1032(w), 1014(w), 1001(w), 948(w), 896(m), 874(w), 845(v s), 793(m), 777(m)$, $752(\mathrm{~m}), 740(\mathrm{~s}), 732(\mathrm{~s}), 713(\mathrm{~m}), 692(\mathrm{~s}), 674(\mathrm{w}), 643(\mathrm{w}), 577(\mathrm{w}), 560(\mathrm{w}), 546(\mathrm{w}), 534$ (w), 495 (w), $480(w), 464$ (w), 441 (s). Raman (633 nm, 10 s, 15 scans, $\left.\mathrm{cm}^{-1}\right): \tilde{v}=3057$ (2), 3051 (2), 3011 (1), 2949 (1), 2919 (2), 2854 (1), 2727 (1), 1613 (4), 1584 (3), 1577 (4), 1567 (2), 1556 (1), 1484 (2), 1467 (2), 1455 (2), 1430 (2), 1417 (3), 1406 (2), 1387 (2), 1382 (2), 1375 (2), 1304 (5), 1297 (4), 1288 (3), 1276 (3), 1245 (1), 1233 (2), 1223 (2), 1189 (2), 1164 (2), 1154 (8), 1135 (2), 1100 (2), 1079 (1), 1049 (4), 1037 (3), 1003 (3), 991 (7), 983 (1), 979 (1), 968 (1), 962 (1), 946 (1), 883 (1), 875 (1), 859 (1), 847 (1), 795 (1), 785 (1), 778 (1), 755 (1), 749 (2), 742 (2), 724 (1), 713 (2), 708 (1), 707 (1), 693 (1), 674 (1), 640 (3), 618 (1), 592 (2), 587 (3), 579 (10), 561 (4), 559 (3), 553 (4), 543 (1), 525 (3), 513 (2), 502 (1), 490 (1), 479 (1), 465 (2), 444 (1), 427 (1), 398 (2), 388 (2), 376 (2), 342 (2), 305 (1), 282 (1), 267 (1), 255 (2), 246 (2), 234 (3). MS (Cl pos., iso-butane) m/z (\%): $93\left[\mathrm{C}_{7} \mathrm{H}_{7}\right]^{+}, 229\left[\mathrm{C}_{61} \mathrm{H}_{58} \mathrm{~N}_{2} \mathrm{P}_{2} \mathrm{~S}_{2}+\mathrm{H}^{+}\right], 315\left[1,3-\text { Mes- } \mathrm{C}_{6} \mathrm{H}_{6}\right]^{+}, 687\left[\mathrm{C}_{37} \mathrm{H}_{33} \mathrm{~N}_{2} \mathrm{P}_{2} \mathrm{~S}_{2}+\right.$ iso-butane $]^{+}, 716\left[[\mathrm{P}(\mu-\mathrm{NTer})]_{2}\right]^{+}, 893\left[\mathrm{C}_{55} \mathrm{H}_{54} \mathrm{~N}_{2} \mathrm{P}_{2} \mathrm{~S}+\text { iso-butane }\right]^{+}$. 
Figure S2: NMR, IR and Raman spectra of $[\mathrm{P}(\mu-\mathrm{NTer})]_{2} \cdot 9 \mathrm{H}$-thioxanthene-9-thione (solvent signals indicated by asterisks).

\section{${ }^{1} \mathrm{H}$ NMR spectrum}
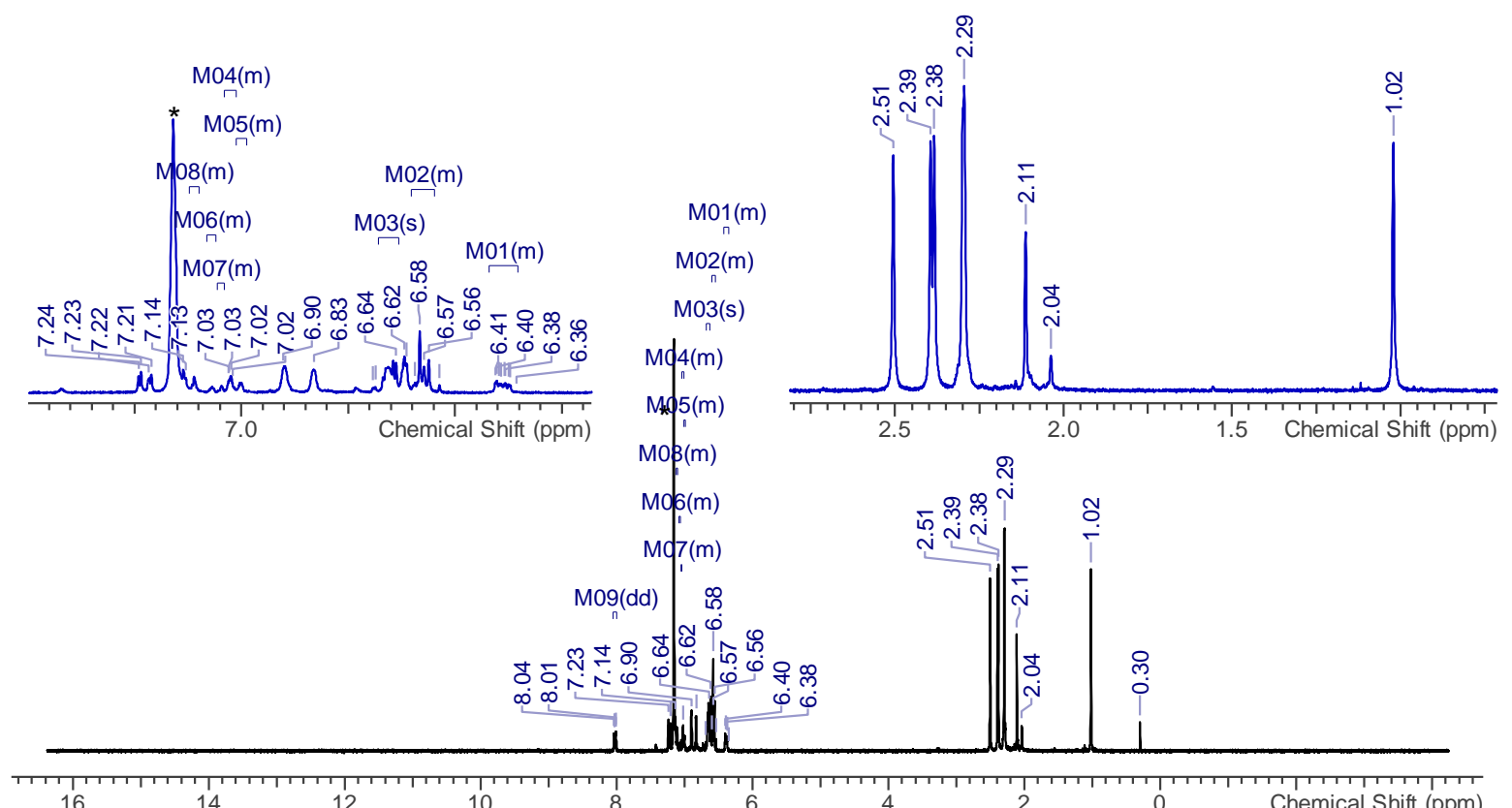

\section{${ }^{13} \mathrm{C}\left\{{ }^{1} \mathrm{H}\right\}$ NMR spectrum}
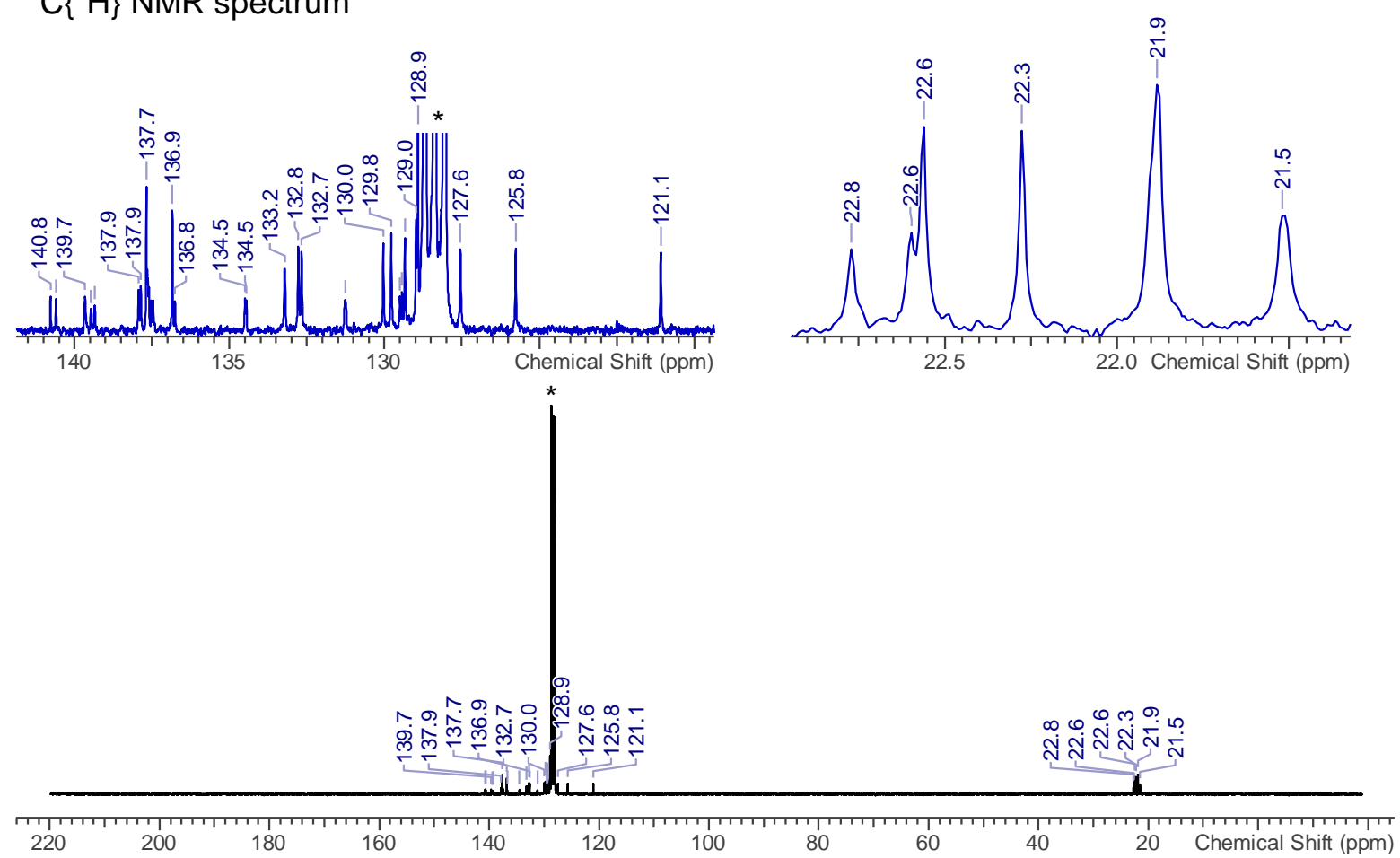
Figure S2: $\mathrm{NMR}$, IR and Raman spectra of $[\mathrm{P}(\mu-\mathrm{NTTe})]_{2} \cdot 9 \mathrm{H}$-thioxanthene-9-thione (solvent signals indicated by asterisks).

${ }^{31} \mathrm{P}\left\{{ }^{1} \mathrm{H}\right\}$ NMR spectrum
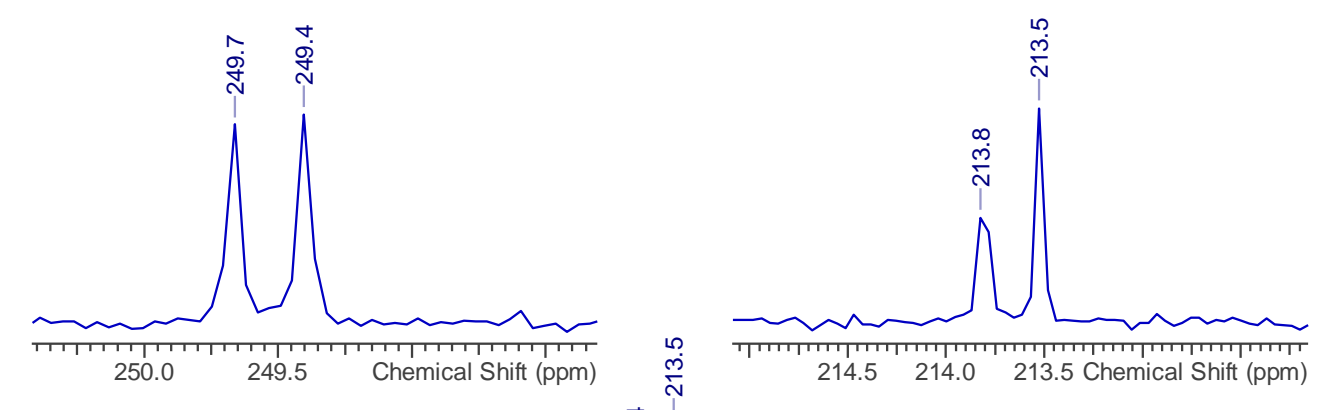

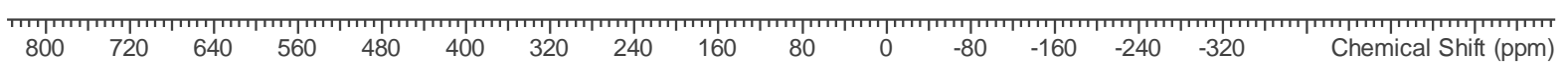

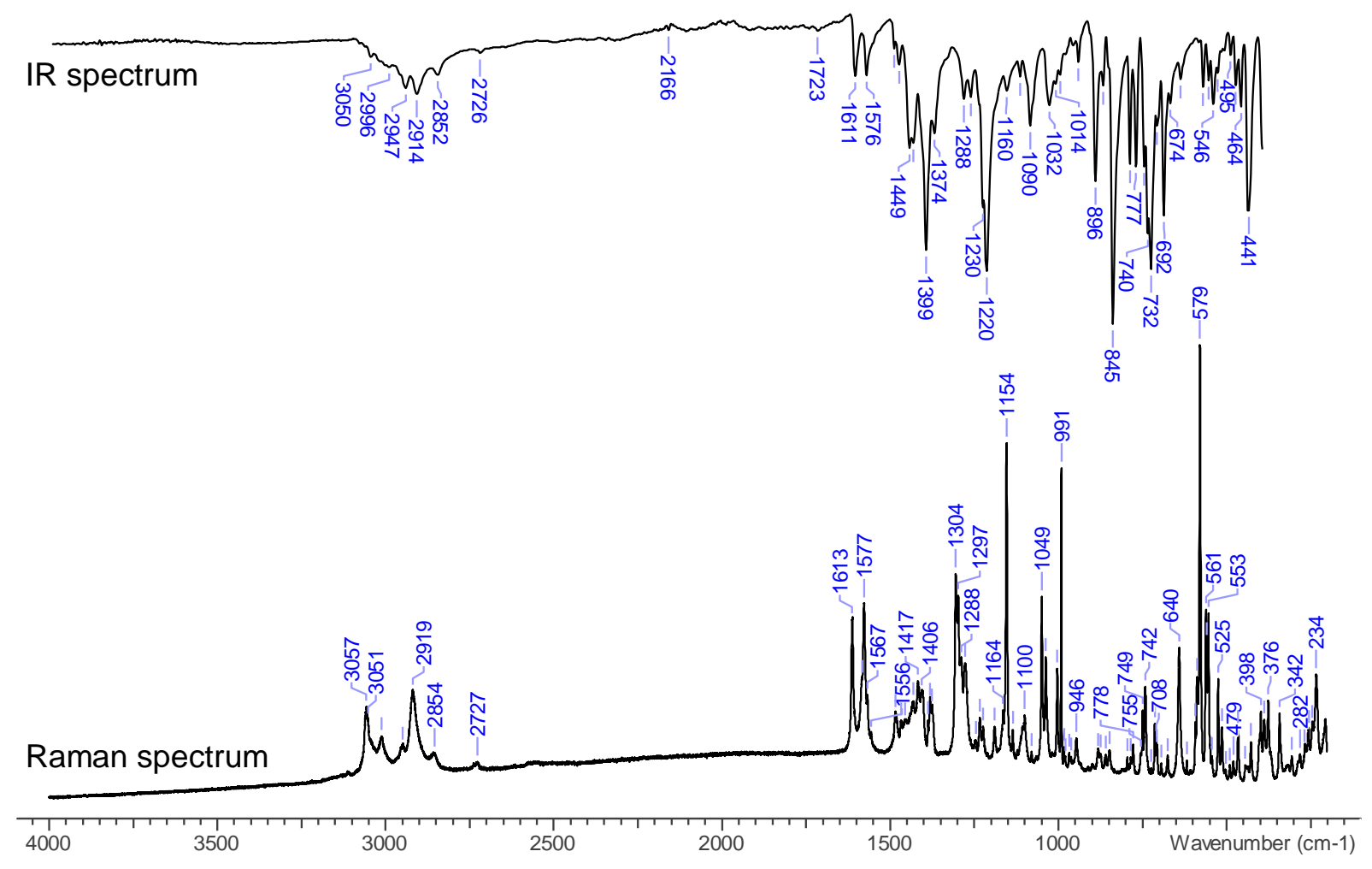




\section{$3.3[\mathrm{P}(\mu-\mathrm{NTer})]_{2} \cdot \mathrm{Fc}-\beta$-naphtyl-thioketone (3Fc)}
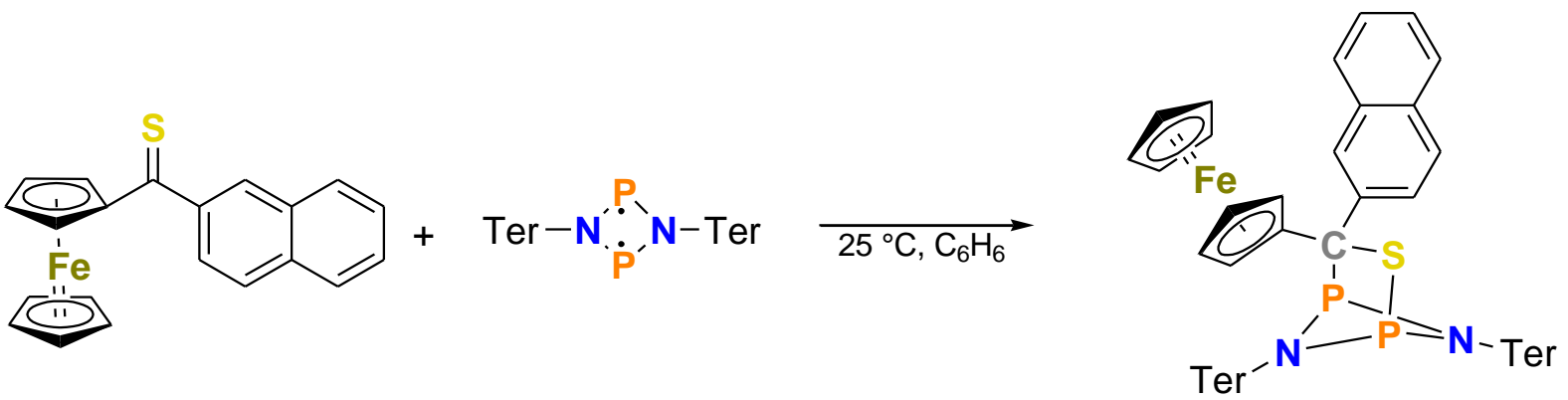

Fc- $\beta$-naphtyl-thioketone $(50 \mathrm{mg}, 0.14 \mathrm{mmol})$ and $[\mathrm{P}(\mu-\mathrm{NTer})]_{2}(100 \mathrm{mg}, 0.14 \mathrm{mmol})$ were combined and dissolved in benzene $(15 \mathrm{~mL})$. An immediate colour change from red to brown was observed. After stirring for one hour, the solution turned purple. Afterwards, all volatile components were removed in vacuo $\left(1 \times 10^{-3} \mathrm{mbar}\right)$ and the purple residue was dried in vacuo $\left(1 \times 10^{-3} \mathrm{mbar}\right)$ for $30 \mathrm{~min}$ at $40^{\circ} \mathrm{C}$ (water bath).

The residue was re-dissolved in toluene $(4 \mathrm{~mL})$. The product was crystallized from a minimal amount of toluene at ambient temperature. Crystals coated with an oily purple layer could be isolated.

Dark yellow crystals of low quality could be grown from a saturated toluene solution at ambient temperature. The adhering violet oily impurities were removed under the microscope.

Separation of the oily violet residue from the crude product was not possible even after washing several times with different solvents. The following analytical data were obtained from the dried, crude product. Only the ${ }^{31} P\left\{{ }^{1} H\right\} N M R$ resonances could be assigned, due to comparison with the isolated substances 305 and 3SS. The raman spectrum was recorded from a crystal cleaned under a microscope.

$\mathbf{C}_{69} \mathbf{H}_{66} \mathbf{N}_{2} \mathbf{P}_{2} \mathbf{S F e}(1073.15 \mathrm{~g} / \mathrm{mol})$. Mp. $206{ }^{\circ} \mathrm{C}-208{ }^{\circ} \mathrm{C}$ (decomp.). CHN calc. (found) in \%: C 77.22 (74.65), H 6.20 (5.94), N 2.61 (2.46), S 2.99 (2.71) (Deviation due to adhering oily impurities). ${ }^{1} \mathbf{H}$ NMR $\left(25{ }^{\circ} \mathrm{C}_{1} \mathrm{C}_{6} \mathrm{D}_{6}, 500.1 \mathrm{MHz}\right): \delta=0.84,1.50,1.64,2.20,2.23,2.32$, 2.35, 2.36, 2.43, 2.45, 2.91, 3.85, 6.41, 6.47, 6.57, 6.63, 6.68, 6.73, 6.87, 6.94, 7.02, 7.30, 7.35, 7.70, 7.79, 7.97, 8.27. ${ }^{13} \mathrm{C}\left\{{ }^{1} \mathrm{H}\right\}$ NMR $\left(25^{\circ} \mathrm{C}, \mathrm{C}_{6} \mathrm{D}_{6}, 125.8 \mathrm{MHz}\right): \delta=21.0,21.1,21.2$, 
21.3, 21.6, 21.8, 21.9, 22.1, 22.5, 22.6, 22.7, 23.4, 67.5, 69.8, 70.1, 72.7, 73.2, 73.6, 121.3, 121.5, 126.0, 126.5, 127.6, 128.9, 129.6, 129.7, 130.5, 130.6, 131.5, 131.8, 133.1, 133.3, 133.4, 133.5, 133.6, 135.9, 136.0, 136.7, 136.8, 137.2, 137.4, 137.5, 138.2, 138.5, 138.8, 139.4, 141.4, 141.5. ${ }^{31} \mathbf{P}\left\{{ }^{1} \mathbf{H}\right\}$ NMR $\left(25^{\circ} \mathrm{C}_{1} \mathrm{C}_{6} \mathrm{D}_{6}, 202.5 \mathrm{MHz}\right): \delta=-19.0,-21.1,95.6$, 199.5, $213.5\left(d, 1 P,{ }^{2} J\left({ }^{31} P_{1}^{31} P\right)=25 \mathrm{~Hz}\right), 227.6,249.1\left(d, 1 P,{ }^{2} J\left({ }^{31} P_{1}^{31} \mathrm{P}\right)=25 \mathrm{~Hz}\right)$. ${ }^{15} \mathbf{N}$ HMBC $\left(25{ }^{\circ} \mathrm{C}, \mathrm{C}_{6} \mathrm{D}_{6}, 50.7 \mathrm{MHz}\right): \delta=-11.2 .{ }^{14} \mathbf{N}$ NMR $\left(25{ }^{\circ} \mathrm{C}, \mathrm{C}_{6} \mathrm{D}_{6}, 18.1 \mathrm{MHz}\right)$ : No signals observed. IR (ATR, 32 scans, cm ${ }^{-1}$ ): $\tilde{v}=3050$ (w), 3027 (w), 2976 (w), 2941 (w), $2912(w), 2852(w), 2726(w), 2695(v w), 1916(v w), 1731(v w), 1609(w), 1578(w), 1572$ (w), $1504(w), 1480(w), 1440(m), 1399(s), 1372(m), 1325(w), 1273(m), 1218(v s), 1158$ (w), $1140(w), 1123(w), 1105(m), 1088(m), 1063(w), 1051(w), 1030(m), 999(m), 950$ (w), $927(w), 905(w), 892(s), 841(v s), 822(s), 810$ s), $791(\mathrm{~s}), 764(w), 748$ (vs), 725 (m), $692(\mathrm{~s}), 641(\mathrm{~m}), 631(\mathrm{w}), 612(\mathrm{~m}), 589(\mathrm{w}), 575(\mathrm{w}), 550(\mathrm{~m}), 521(\mathrm{w}), 497(\mathrm{~m}), 482(\mathrm{~s})$, 466 (m), 451 (s), 435 (s), 406 (s). Raman (633 nm, 10 s, 15 scans, cm ${ }^{-1}$ ): $\tilde{v}=1612$ (1), 1582 (1), 1573 (1), 1486 (1), 1427 (1), 1411 (1), 1381 (1), 1311 (1), 1301 (1), 1275 (1), 1272 (1), 1245 (1), 1221 (3), 1208 (1), 1198 (3), 1192 (2), 1158 (1), 1122 (1), 1107 (8), 1094 (1), 1050 (1), 1035 (1), 1019 (1), 1001 (1), 946 (1), 915 (1), 894 (1), 885 (1), 856 (1), 847 (1), 808 (1), 793 (1), 773 (1), 766 (1), 754 (2), 741 (2), 723 (2), 704 (1), 642 (2), 612 (1), 590 (3), 582 (8), 559 (3), 548 (4), 533 (1), 519 (1), 509 (1), 497 (1), 481 (1), 467 (2), 452 (1), 427 (1), 416 (2), 396 (1), 384 (3), 334 (1), 317 (3), 307 (2), 286 (1), 270 (2), 253 (3), 236 (4), 211 (3), 182 (3), 169 (3), 151 (2), 137 (4), 121 (7), 101 (6), 91 (10), 80 (8). MS (Cl pos., iso-butane) m/z (\%): 69, $92\left[\mathrm{C}_{7} \mathrm{H}_{7}\right]^{+}, 328[\text { Ter- } \mathrm{NH}]^{+\cdot}, 330\left[\text { Ter- } \mathrm{NH}_{3}\right]^{+}, 357$ $\left[\mathrm{C}_{21} \mathrm{H}_{16} \mathrm{FeS}\right]^{+}, 386 \quad\left[\text { Ter- } \mathrm{NH}_{3} \text { iso-butane }\right]^{+}, 413\left[\mathrm{C}_{21} \mathrm{H}_{16} \mathrm{FeS}\right.$ iso-butane ${ }^{+}, 716$ $\left[[\mathrm{P}(\mu-\mathrm{NTer})]_{2}\right]^{+}, 767,1007\left[\mathrm{C}_{64} \mathrm{H}_{61} \mathrm{~N}_{2} \mathrm{P}_{2} \mathrm{SFe}\right]^{+}, 1074[\mathrm{M}]^{+}$. 
Figure S3: $\mathrm{NMR}, \mathrm{IR}$ and Raman spectra of $[\mathrm{P}(\mu-\mathrm{NTer})]_{2} \cdot \mathrm{Fc}-\beta$-naphtyl-thioketone (solvent signals indicated by asterisks).

${ }^{1} \mathrm{H}$ NMR spectrum




Figure S3: $\mathrm{NMR}, \mathrm{IR}$ and Raman spectra of $[\mathrm{P}(\mu-\mathrm{NTer})]_{2} \cdot \mathrm{Fc}-\beta$-naphtyl-thioketone (solvent signals indicated by asterisks).

${ }^{31} \mathrm{P}\left\{{ }^{1} \mathrm{H}\right\}$ NMR spectrum

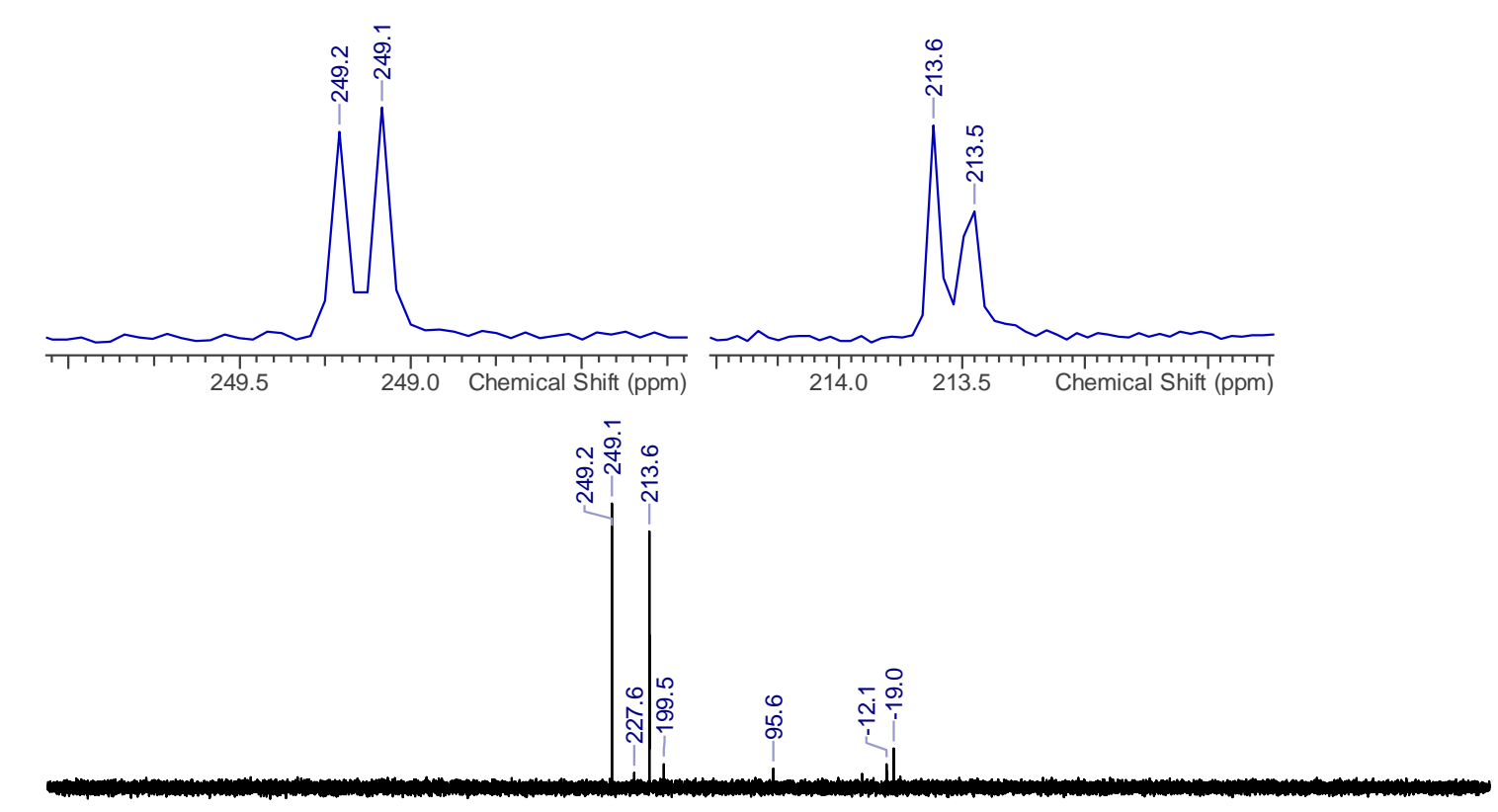

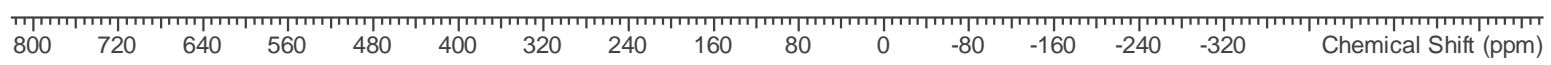
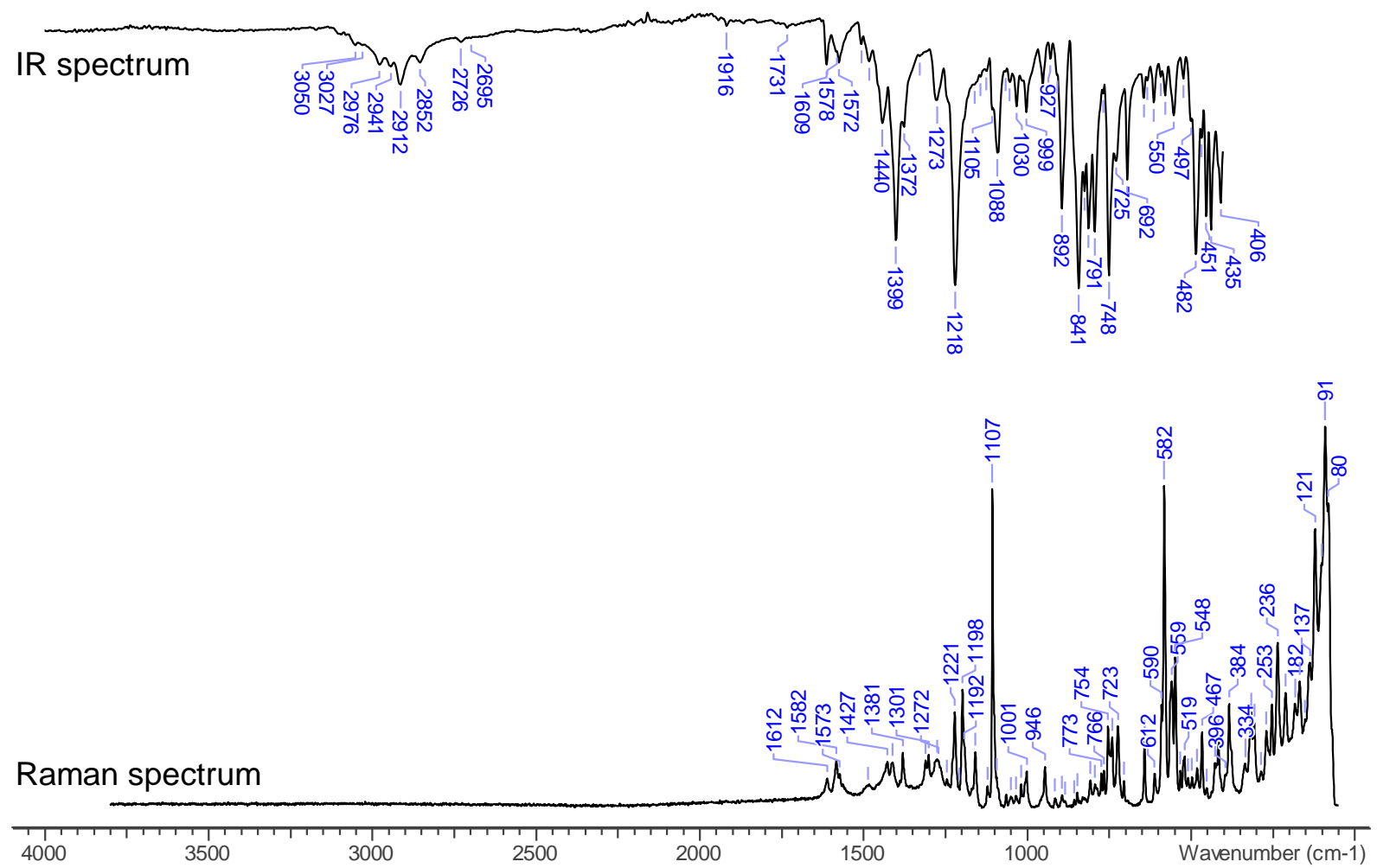


\subsection{Attempted synthesis of $[\mathrm{P}(\mu-\mathrm{NTer})]_{2} \cdot 9 \mathrm{H}$-xanthene-9-one}<smiles>O=c1c2ccccc2oc2[14c]1[CH+]C=C2</smiles>
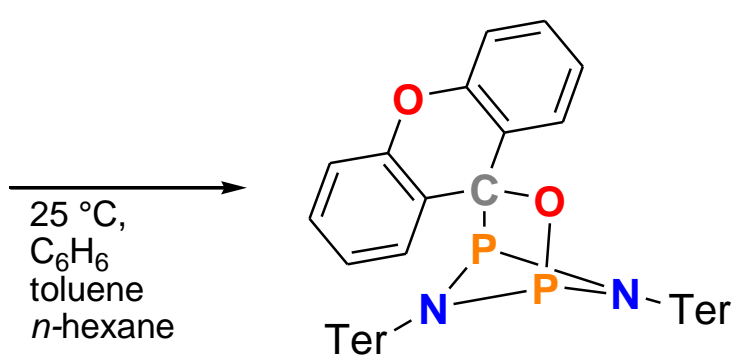

9H-xanthene-9-one $(11 \mathrm{mg}, 0.05 \mathrm{mmol})$ and $[\mathrm{P}(\mu-\mathrm{NTer})]_{2}(32 \mathrm{mg}, 0.04 \mathrm{mmol})$ were combined in an Young-NMR-tube and dissolved in toluene- $d_{8}(0.5 \mathrm{~mL})$. No color change or reaction could be observed. In the ${ }^{31} \mathrm{P}\left\{{ }^{1} \mathrm{H}\right\}$ NMR spectrum, only signals of the starting materials could be detected.

9H-xanthene-9-one $(11 \mathrm{mg}, 0.05 \mathrm{mmol})$ and $[\mathrm{P}(\mu-\mathrm{NTer})]_{2}(32 \mathrm{mg}, 0.04 \mathrm{mmol})$ were combined in an Young-NMR-tube and dissolved in $n$-hexane $(0.5 \mathrm{~mL})$. No color change or reaction could be observed. In the ${ }^{31} \mathrm{P}\left\{{ }^{1} \mathrm{H}\right\}$ NMR spectrum, only signals of the starting materials could be detected.

9H-xanthene-9-one $(42 \mathrm{mg}, 0.21 \mathrm{mmol})$ and $[\mathrm{P}(\mu-\mathrm{NTer})]_{2}(10 \mathrm{mg}, 0.01 \mathrm{mmol})$ were combined in an Young-NMR-tube and dissolved in toluene- $d_{8}(0.5 \mathrm{~mL})$. The solution was degassed by several freeze-pump-thaw cycles. Afterwards the reaction mixture was stored at $80{ }^{\circ} \mathrm{C}$ for 70 hours (drying oven). No color change or reaction could be observed. In the ${ }^{31} \mathrm{P}\left\{{ }^{1} \mathrm{H}\right\}$ NMR spectrum, only signals of the starting materials could be detected. 


\subsection{Reaction with Hydrogen}<smiles>S=c1c2ccccc2oc2ccccc12</smiles>

[P( $\mu$-NTer $]_{2} \cdot 9 \mathrm{H}$-xanthene-9-thione $(190 \mathrm{mg}, 0.20 \mathrm{mmol})$ was dissolved in toluene $(20 \mathrm{~mL})$ in a $50 \mathrm{~mL}$ two-neck Schlenk flask equipped with a gas bubbler and gas inlet tube. $\mathrm{H}_{2}$ gas was bubbled trough the stirred reaction mixture at ambient temperature. The greenish reaction mixture turned bluish within $5 \mathrm{~min}$. After $50 \mathrm{~min}$ the gas flow was stopped. The reaction solution was analyzed by ${ }^{31} \mathrm{P}\left\{{ }^{1} \mathrm{H}\right\}$ NMR spectroscopy and showed the literature known signals of $[\mathrm{P}(\mu-\mathrm{NTer})]_{2} \cdot \mathrm{H}_{2}{ }^{[7]}$

Afterwards all volatile were removed in vacuo $\left(1 \times 10^{-3} \mathrm{mbar}\right)$ and the residue was dried in vacuo $\left(1 \times 10^{-3} \mathrm{mbar}\right)$ for $90 \mathrm{~min}$ at ambient temperature. The residue was redissolved in toluene $(4 \mathrm{~mL})$. Pure $9 \mathrm{H}$-xanthene-9-thione could be crystallized from a minimal amount of toluene at ambient temperature. 


\subsection{High-temperature NMR spectra of $[\mathrm{P}(\mu-\mathrm{NTer})]_{2} \cdot 9 \mathrm{H}$-xanthene-9- thione (3OS)}
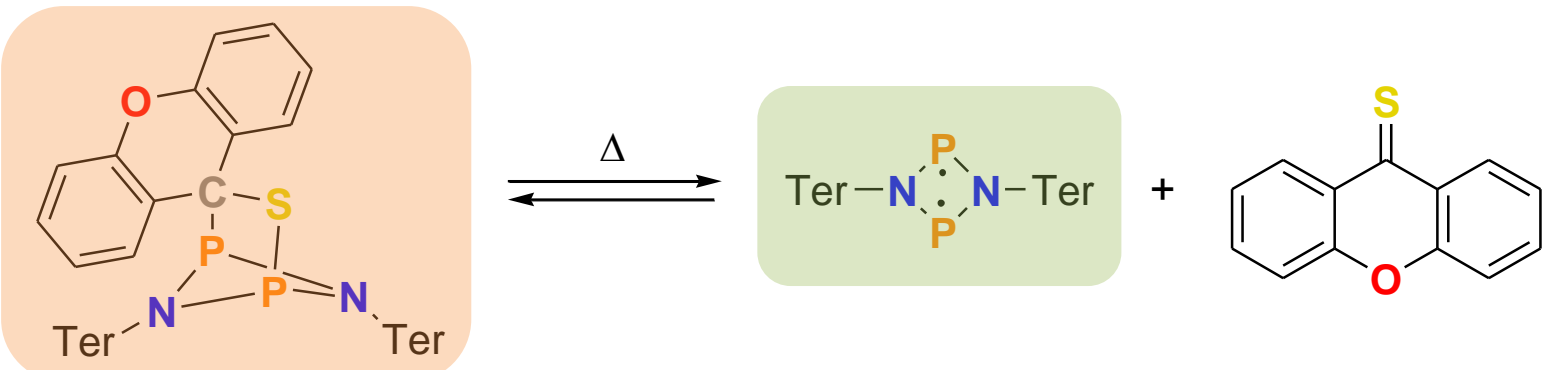

[P( $\mu$-NTer) $]_{2} \cdot 9 \mathrm{H}$-xanthene-9-thione $(11 \mathrm{mg}, 0.05 \mathrm{mmol})$ was filled in a Young-NMRtube and was dissolved in toluene- $d_{8}(0.5 \mathrm{~mL})$. The solution was degassed by several freeze-pump-thaw cycles. When the temperature was increased from $25^{\circ} \mathrm{C}$ to $100{ }^{\circ} \mathrm{C}$, a colour change from greenish-yellow to red was observed and the signals of the adduct $[\mathrm{P}(\mu \text {-NTer })]_{2} \cdot 9 \mathrm{H}$-xanthene-9-thione decreased and the signal of the 4-membered biradical $[\mathrm{P}(\mu-\mathrm{NTer})]_{2}$ increased. Upon cooling the solution to room temperature, the signals of $\mathbf{3 0 S}$ were recovered.

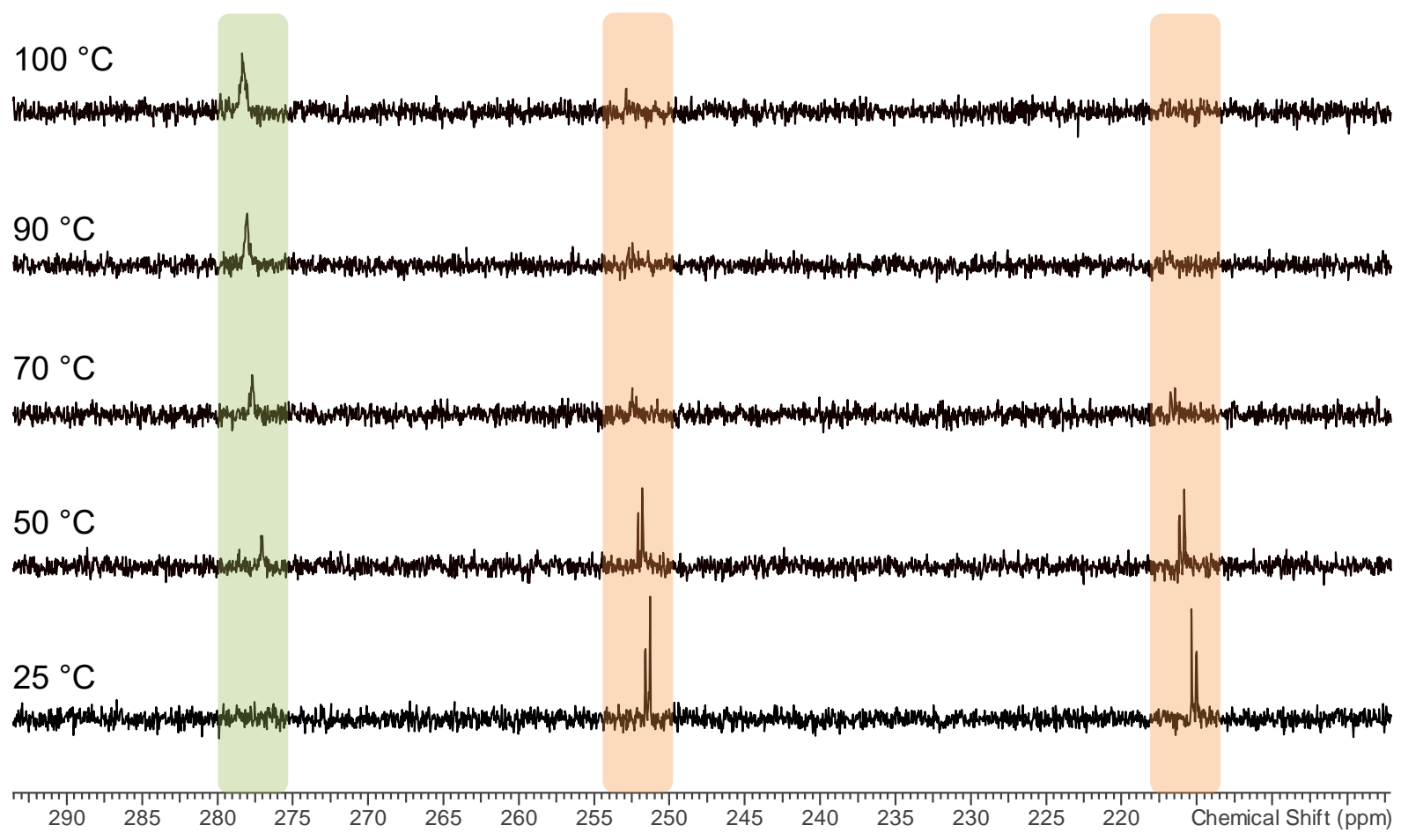


The same NMR experiment was carried out for the derivative 3SS. However, due to its very low solubility, the NMR spectra display a very low signal-to-noise ratio. The doublet signals of $3 \mathrm{SS}$ disappear at a temperature of about $80^{\circ} \mathrm{C}$. However, the signal of $\mathbf{1 T e r}$ is barely detectable.

$$
100^{\circ} \mathrm{C}
$$

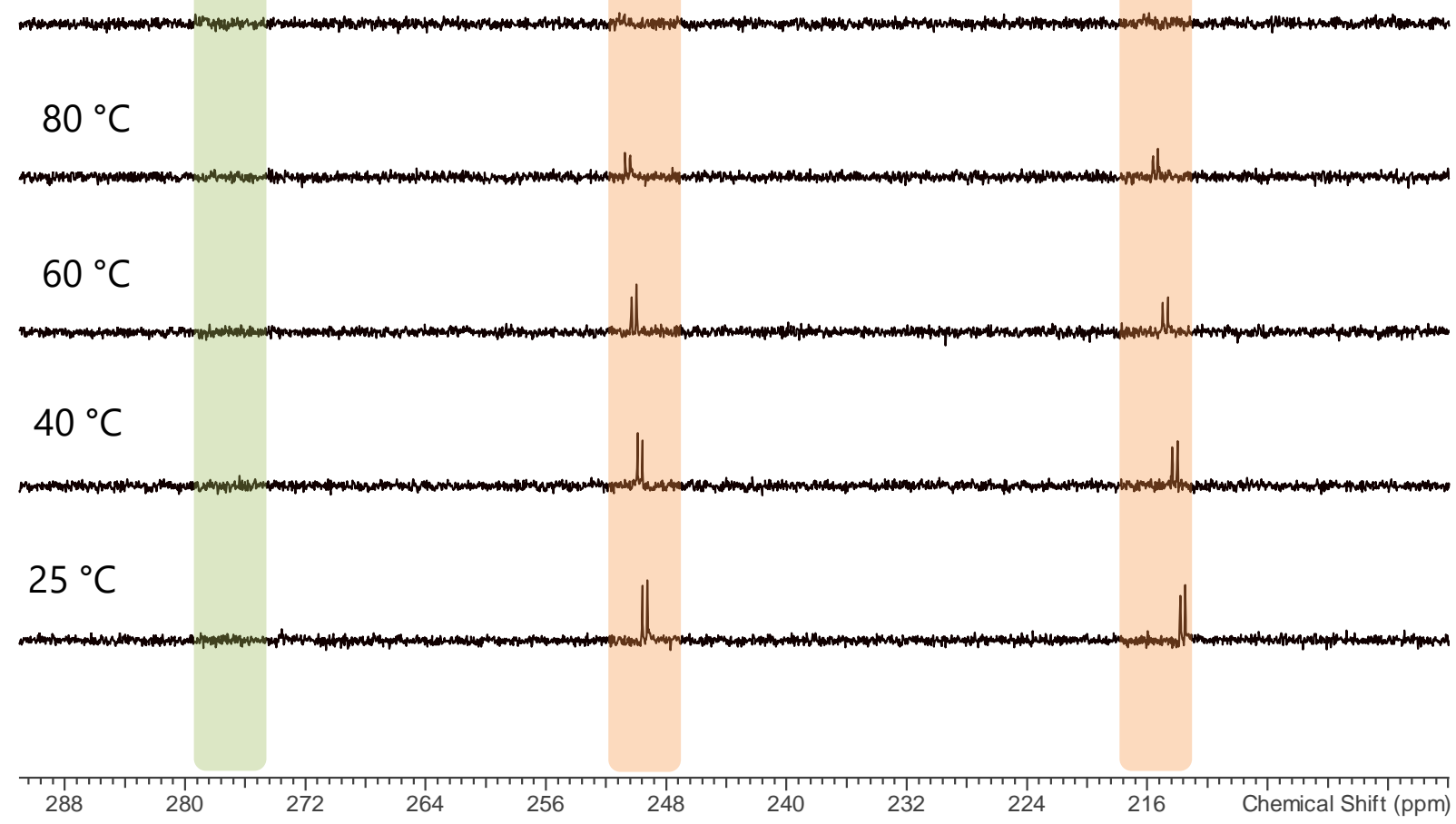




\section{Additional spectroscopic details}

\section{1 ${ }^{31} \mathrm{P}\left\{{ }^{1} \mathrm{H}\right\}$ NMR data of the adduct species}

All three derivatives of type $\mathbf{3}$ are poorly soluble in all common solvents. Therefore, the evaluation of the NMR spectra was difficult. The following tables contain experimental ${ }^{31} \mathrm{P}\left\{{ }^{1} \mathrm{H}\right\}$ NMR data. Calculated values are given in brackets for comparison (GIAO method, PBE-D3/def2-TZVP).

Table S3. ${ }^{31} \mathrm{P}\left\{{ }^{1} \mathrm{H}\right\}$ NMR data of $[\mathrm{P}(\mu-\mathrm{NTer})]_{2} \cdot 9 \mathrm{H}$-xanthene-9-thione.

\begin{tabular}{|c|c|c|c|}
\hline & $\delta$ [ppm] & $J[\mathrm{~Hz}]$ & \\
\hline$A$ & $+251.5(+298.8)$ & & \\
\hline$x$ & $+215.0(+249.2)$ & $J_{A X}=33(21)$ & $=\mathbf{N}_{\text {Ter }}$ \\
\hline
\end{tabular}

Table S4. ${ }^{31} \mathrm{P}\left\{{ }^{1} \mathrm{H}\right\} \mathrm{NMR}$ data of $[\mathrm{P}(\mu-\mathrm{NTer})]_{2} \cdot 9 H$-thioxanthene-9-thione.

\begin{tabular}{|c|c|c|c|}
\hline & $\delta$ [ppm] & $J[\mathrm{~Hz}]$ & \\
\hline$A$ & $+249.4(+295.5)$ & & \\
\hline$x$ & $+213.5(+246.7)$ & $J_{\mathrm{AX}}=31(26)$ & $=\mathbf{N}_{\text {Ter }}$ \\
\hline
\end{tabular}

Table S5. ${ }^{31} \mathrm{P}\left\{{ }^{1} \mathrm{H}\right\}$ NMR data of $[\mathrm{P}(\mu$-NTer $)] 2 \cdot \mathrm{Fc}-\beta$-naphtyl-thioketone.

\begin{tabular}{|c|c|c|c|}
\hline & $\delta$ [ppm] & $J[\mathrm{~Hz}]$ & \\
\hline A & $+249.1(+296.4)$ & & \\
\hline$x$ & $+213.5(+250.6)$ & $J_{A X}=25(19)$ & \\
\hline
\end{tabular}




\section{Computational details}

\subsection{General remarks}

Computations were carried out using Gaussian09 ${ }^{[8]}$ or ORCA 4.2.1. ${ }^{[9]}$

Structure optimizations employed the pure DFT exchange-correlation functional $P B E^{[10,11]}$ in conjunction with Grimme's dispersion correction $D 3(B J)^{[12,13]}$ and the def2TZVP basis set ${ }^{[14]}$ (notation PBE-D3/def2-TZVP). The resolution of identity (RI) approximation was employed, using Weigend's accurate Coulomb fitting basis. ${ }^{[15]}$ All structures were fully optimized and confirmed as minima by frequency analyses. Chemical shifts and coupling constants were derived by the GIAO method at the PBED3/def2-TZVP level of theory. ${ }^{[16-21]}$ The calculated absolute shifts $\left(\sigma_{\text {calc,x }}\right)$ were referenced to the experimental absolute shift of $85 \% \mathrm{H}_{3} \mathrm{PO}_{4}$ in the gas phase $\left(\sigma_{\text {ref }, 1}=328.35 \mathrm{ppm}\right)^{[22]}$ using $\mathrm{PH}_{3}\left(\sigma_{\mathrm{ref}, 2}=594.45 \mathrm{ppm}\right)$ as a secondary standard: ${ }^{[23]}$

$$
\begin{aligned}
\delta_{\text {calc, }, X} & =\left(\sigma_{\text {ref, },}-\sigma_{\text {ref, }, 2}\right)-\left(\sigma_{\text {calc }, \mathrm{X}}-\sigma_{\text {calc, }, \mathrm{PH}_{3}}\right) \\
& =\sigma_{\text {calc, } \mathrm{PH}_{3}}-\sigma_{\text {calc, },}-266.1 \mathrm{ppm}
\end{aligned}
$$

At the PBE-D3/def2-TZVP level of theory, $\sigma_{\mathrm{cal}, \mathrm{PH}_{3}}$ amounts to $+563.04 \mathrm{ppm}$.

More accurate electronic energies for optimized structures were computed by singlepoint DLPNO-CCSD $\left(\mathrm{T}^{[24-27]}\right.$ calculations employing the def2-TZVP basis set ${ }^{[14]}$ and def2-TZVP/C correlation fitting basis ${ }^{[28]}$ (notation: DLPNO-CCSD(T)/def2-TZVP//PBED3/def2-TZVP). Thermodynamic quantities at this level of theory were calculated using the DLPNO-CCSD $(T)$ single point energy and the thermal corrections at the PBED3/def2-TZVP level of theory. The $T_{1}$ diagnostic was evaluated to ensure reliable results (empirically, $\operatorname{CCSD}(T)$ results are considered reliable if $\left.T_{1}<0.02\right) .{ }^{[29]}$

To account for solvent effects, the Gibbs free energies of solvation were computed using the SMD continuum solvation mode ${ }^{[30]}$ at the PBE-D3/def2-TZVP level of theory. 


\subsection{Summary of calculated data}

For the sake of clarity, the following compounds are abbreviated as follows:

Table S6. Abbreviations.

\begin{tabular}{|c|c|}
\hline Compd. & Abbreviation \\
\hline $9 H$-xanthene-9-one & $\mathrm{OO}$ \\
\hline 9H-xanthene-9-thione & OS \\
\hline 9H-thioxanthene-9-thione & SS \\
\hline Fc- $\beta$-naphtyl-thioketone & $\mathrm{Fc}$ \\
\hline acetone & $\mathrm{CO}$ \\
\hline thioacetone & CS \\
\hline$[\mathrm{P}(\mu \text {-NTer })]_{2} \cdot 9 \mathrm{H}$-xanthene-9-thione & {$[\mathrm{P}(\mu \text {-NTer })]_{2} \cdot \mathrm{SO}$} \\
\hline$[\mathrm{P}(\mu \text {-NTer })]_{2} \cdot 9 \mathrm{H}$-thioxanthene-9-thione & {$[\mathrm{P}(\mu-\mathrm{NTer})]_{2} \cdot \mathrm{SS}$} \\
\hline$[\mathrm{P}(\mu \text {-NTer })]_{2} \cdot 9 \mathrm{H}$-xanthene-9-one & {$[\mathrm{P}(\mu-\mathrm{NTer})]_{2} \cdot \mathrm{OO}$} \\
\hline$[\mathrm{P}(\mu \text {-NTer })]_{2} \cdot \mathrm{Fc}-\beta$-naphtyl-thioketone & {$[\mathrm{P}(\mu-\mathrm{NTer})]_{2} \cdot \mathrm{Fc}$} \\
\hline$[\mathrm{P}(\mu \text {-NTer })]_{2} \cdot$ acetone & {$[\mathrm{P}(\mu \text {-NTer })]_{2} \cdot \mathrm{CO}$} \\
\hline$[\mathrm{P}(\mu \text {-NTer })]_{2}$ thioacetone & {$[\mathrm{P}(\mu-\mathrm{NTer})]_{2} \cdot \mathrm{CS}$} \\
\hline
\end{tabular}

\subsubsection{Thermodynamic data}

Table S7. Summary of calculated data, including electronic energies and thermal corrections.

\begin{tabular}{|c|c|c|c|c|c|c|}
\hline Compd. & PG & $\begin{array}{c}\text { Opt. } \\
\text { method }\end{array}$ & $E_{\text {tot }}{ }^{[\mathrm{aa}]}$ & $\Delta G^{[b]}$ & $E_{\operatorname{CCSD}(T)^{[C]}}$ & $T_{1}$ \\
\hline$[\mathrm{P}(\mu \text {-NTer })]_{2}$ & $D_{2}$ & \multirow{11}{*}{$\begin{array}{l}\text { PBE-D3/ } \\
\text { def2-TZVP }\end{array}$} & -2649.5088 & 0.7310 & -2646.8678 & 0.010 \\
\hline OS & $C_{2 v}$ & & -972.9980 & 0.1304 & -972.0969 & 0.014 \\
\hline SS & $C_{S}$ & & -1295.8547 & 0.1251 & -1294.6967 & 0.014 \\
\hline OO & $C_{2 v}$ & & -650.1518 & 0.1336 & -649.3277 & 0.011 \\
\hline Fc & $C_{1}$ & & -2470.7702 & 0.2474 & -2469.9384 & 0.025 \\
\hline $\mathrm{CO}$ & $C_{2 v}$ & & -192.99388 & 0.0519 & -192.8233 & 0.025 \\
\hline CS & $C_{2 v}$ & & -515.8337 & 0.0510 & -515.4074 & 0.015 \\
\hline$[\mathrm{P}(\mu \text {-NTer })]_{2} \cdot \mathrm{OS}$ & $C_{1}$ & & -3622.5524 & 0.8955 & -3619.0180 & 0.010 \\
\hline$[\mathrm{P}(\mu-\mathrm{NTer})]_{2} \cdot \mathrm{SS}$ & $C_{1}$ & & -3945.4129 & 0.8924 & -3941.6239 & 0.010 \\
\hline$[\mathrm{P}(\mu \text {-NTer })]_{2} \cdot \mathrm{OO}$ & $C_{1}$ & & -3299.6866 & 0.8982 & -3296.4119 & 0.010 \\
\hline$[\mathrm{P}(\mu-\mathrm{NTer})]_{2} \cdot \mathrm{CO}$ & $C_{1}$ & & -2842.5453 & 0.8151 & -2839.7365 & 0.013 \\
\hline
\end{tabular}




\begin{tabular}{|c|c|c|c|c|c|c|}
\hline Compd. & PG & $\begin{array}{c}\text { Opt. } \\
\text { method }\end{array}$ & $E_{\text {tot }}{ }^{[a]}$ & $\Delta G^{[b]}$ & $E_{\mathrm{CCSD}(\mathrm{T})}{ }^{[\mathrm{c}]}$ & $T_{1}$ \\
\hline$[\mathrm{P}(\mu \text {-NTer })]_{2} \cdot \mathrm{CS}$ & $C_{1}$ & & -3165.4083 & 0.8129 & -3162.3400 & 0.010 \\
\hline$[\mathrm{P}(\mu-\mathrm{NTer})]_{2} \cdot \mathrm{Fc}$ & $C_{1}$ & & -5120.3381 & 1.0138 & -5115.8706 & 0.016 \\
\hline
\end{tabular}

[a] Total SCF energy in a.u.; [b] thermal correction to Gibbs energy in a.u. (298 K unless stated otherwise); [c] single-point DLPNO-CCSD(T)/def2-TZVP energy.

\subsubsection{Reaction energies}

Table S8. Reaction energies (DLPNO-CCSD(T)/def2-TZVP//PBE-D3/def2-TZVP, in $\mathrm{kJ} \cdot \mathrm{mol}^{-1}, \mathrm{c}^{\circ}=1 \mathrm{~mol} / \mathrm{L}$, solvent $=$ toluene).

\begin{tabular}{|c|c|c|c|c|}
\hline Reaction & $\Delta E^{\text {tot }}$ & $\Delta_{\mathrm{R}} H$ & $\Delta_{\mathrm{R}} \mathbf{G}^{\circ}$ & $\Delta_{\mathrm{R}} \boldsymbol{G}_{\text {solv }}{ }^{\circ}$ \\
\hline$[\mathrm{P}(\mu-\mathrm{NTer})]_{2}+\mathrm{OS} \rightarrow[\mathrm{P}(\mu-\mathrm{NTer})]_{2} \cdot \mathrm{OS}$ & -139.98 & -133.91 & -58.48 & -30.05 \\
\hline$[\mathrm{P}(\mu-\mathrm{NTer})]_{2}+\mathrm{SS} \rightarrow[\mathrm{P}(\mu-\mathrm{NTer})]_{2} \cdot \mathrm{SS}$ & -155.94 & -149.12 & -68.86 & -41.00 \\
\hline$[\mathrm{P}(\mu-\mathrm{NTer})]_{2}+\mathrm{OO} \rightarrow[\mathrm{P}(\mu-\mathrm{NTer})]_{2} \cdot \mathrm{OO}$ & -93.23 & -87.54 & -13.20 & +14.23 \\
\hline$[\mathrm{P}(\mu-\mathrm{NTer})]_{2}+\mathrm{CO} \rightarrow[\mathrm{P}(\mu-\mathrm{NTer})]_{2} \cdot \mathrm{CO}$ & -119.07 & -111.44 & -42.55 & -22.45 \\
\hline$[\mathrm{P}(\mu-\mathrm{NTer})]_{2}+\mathrm{CS} \rightarrow[\mathrm{P}(\mu-\mathrm{NTer})]_{2} \cdot \mathrm{CS}$ & -170.00 & -162.21 & -96.78 & -76.27 \\
\hline$[\mathrm{P}(\mu-\mathrm{NTer})]_{2}+\mathrm{Fc} \rightarrow[\mathrm{P}(\mu-\mathrm{NTer})]_{2} \cdot \mathrm{Fc}$ & -169.16 & -162.84 & -84.17 & -51.29 \\
\hline
\end{tabular}

\subsubsection{Distortion energies}

\section{thioketone}
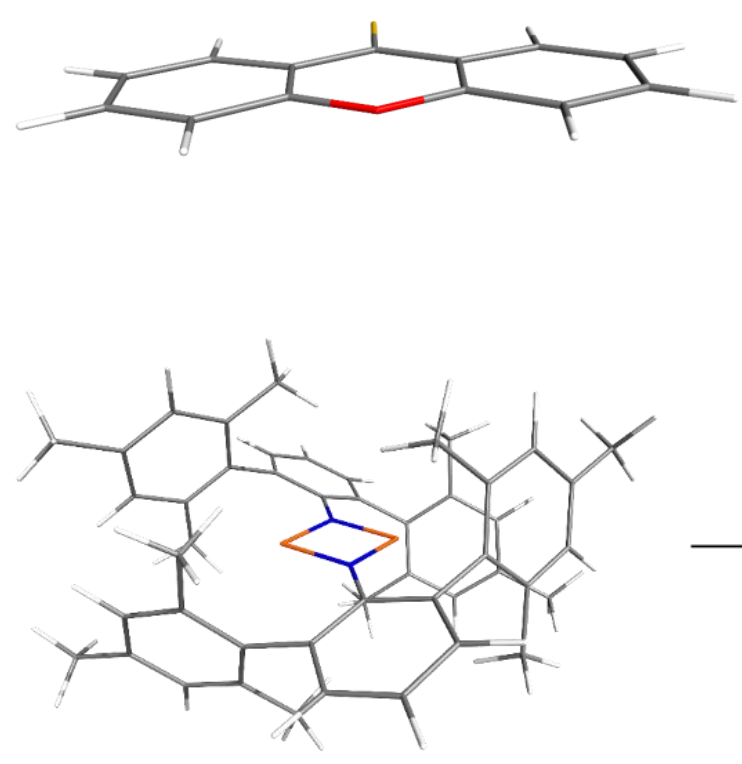

thioketone dis

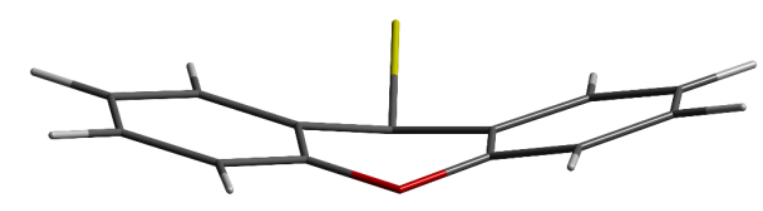

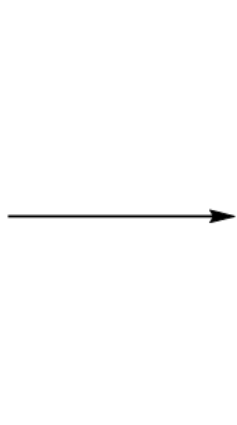

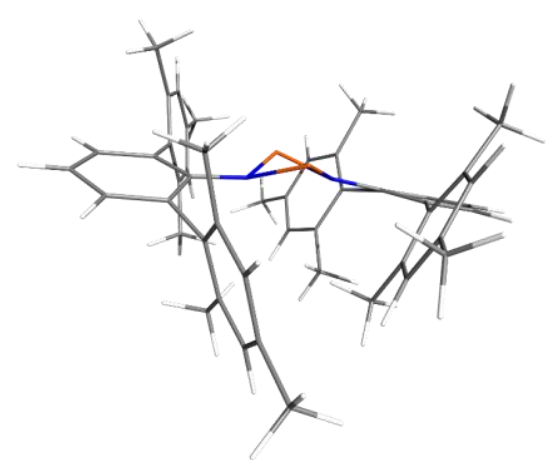


To calculate the energies of the distorted compounds, the structural parameters of the thioketones or biradical moieties were taken from the corresponding adduct structures and a single point calculation was performed on this distorted structure. The distortion is shown for the SO derivative. The distortion energies are presented in Table S9.

Table 59. Distortion energies ${ }^{[a]}$ (DLPNO-CCSD(T)/def2-TZVP//PBE-D3/def2-TZVP) for the reaction of $\mathbf{1 T e r}+\mathbf{2 X Y} \rightarrow \mathbf{3 X Y}$ along with the interaction energy ${ }^{[\mathrm{b}]} \Delta \mathrm{E}^{\mathrm{int}}$. The acetone and thioacetone derivates (3Ac, 3SAc) were added for comparison.

\begin{tabular}{|c|c|c|c|c|}
\hline $\mathbf{X Y}$ & $\Delta E_{B}^{\text {dist }}$ & $\Delta E_{X Y}{ }^{\text {dist }}$ & $\Sigma \Delta E^{\text {dist }}$ & $\Delta E^{\text {int }}$ \\
\hline SS & 168.2 & 158.1 & 326.3 & -482.2 \\
\hline OS & 165.7 & 161.7 & 327.4 & -467.4 \\
\hline 00 & 262.7 & 234.4 & 497.0 & -590.3 \\
\hline Ac & 242.6 & 243.4 & 485.9 & -605.0 \\
\hline SAC & 149.1 & 154.3 & 303.5 & -473.5 \\
\hline Fc & 168.0 & 165.7 & 333.7 & -502.9 \\
\hline
\end{tabular}




\subsection{Optimized structures (.xyz-files)}

\subsection{1 $\mathrm{PH}_{3}$}

$\begin{array}{lcrr}4 & & & \\ \mathrm{PH3} & 0 . \mathrm{PBE}-\mathrm{D} 3 / \mathrm{def} 2-\mathrm{TZVP} & & \\ \mathrm{P} & 0.00000 & 0.00000 & 0.13202 \\ \mathrm{H} & 0.00000 & 1.19102 & -0.66012 \\ \mathrm{H} & -1.03145 & -0.59551 & -0.66012 \\ \mathrm{H} & 1.03145 & -0.59551 & -0.66012\end{array}$

\subsubsection{0}

\begin{tabular}{|c|c|c|c|}
\hline \multicolumn{4}{|c|}{$\begin{array}{l}23 \\
00 @ P B E-D 3 / \text { def2-TZVP }\end{array}$} \\
\hline C & 0.00000 & 3.58177 & -0.97074 \\
\hline C & 0.00000 & 2.34869 & -1.60931 \\
\hline C & 0.00000 & 1.18164 & -0.83578 \\
\hline C & 0.00000 & 1.23865 & 0.56907 \\
\hline C & 0.00000 & 2.50142 & 1.18841 \\
\hline C & 0.00000 & 3.66303 & 0.43249 \\
\hline C & 0.00000 & 0.00000 & 1.37191 \\
\hline C & -0.00000 & -1.23865 & 0.56907 \\
\hline C & -0.00000 & -1.18164 & -0.83578 \\
\hline C & -0.00000 & -2.34869 & -1.60931 \\
\hline $\mathrm{H}$ & 0.00000 & -2.26387 & -2.69624 \\
\hline C & -0.00000 & -3.58177 & -0.97074 \\
\hline C & -0.00000 & -3.66303 & 0.43249 \\
\hline C & -0.00000 & -2.50142 & 1.18841 \\
\hline $\mathrm{H}$ & 0.00000 & 4.49407 & -1.56917 \\
\hline $\mathrm{H}$ & 0.00000 & 2.26387 & -2.69624 \\
\hline $\mathrm{H}$ & 0.00000 & 2.52122 & 2.27931 \\
\hline $\mathrm{H}$ & 0.00000 & 4.63767 & 0.92147 \\
\hline $\mathrm{H}$ & -0.00000 & -4.49407 & -1.56917 \\
\hline $\mathrm{H}$ & -0.00000 & -4.63767 & 0.92147 \\
\hline $\mathrm{H}$ & -0.00000 & -2.52122 & 2.27931 \\
\hline 0 & 0.00000 & 0.00000 & 2.60468 \\
\hline 0 & 0.00000 & 0.00000 & -1.52867 \\
\hline
\end{tabular}

\subsubsection{OS}

$\begin{array}{lccr}23 & & & \\ \text { SO @ PBE-D3/def2-TZVP } & & \\ \text { C } & -0.00000 & 3.57712 & -1.23947 \\ \text { C } & -0.00000 & 2.33749 & -1.86081 \\ \text { C } & -0.00000 & 1.17844 & -1.07519 \\ \text { C } & -0.00000 & 1.23200 & 0.33457 \\ \text { C } & -0.00000 & 2.51221 & 0.92964 \\ \text { C } & -0.00000 & 3.66456 & 0.16395 \\ \text { C } & 0.00000 & 0.00000 & 1.12504\end{array}$




$\begin{array}{lrrr}C & -0.00000 & -1.23200 & 0.33457 \\ C & -0.00000 & -1.17844 & -1.07519 \\ C & -0.00000 & -2.33749 & -1.86081 \\ H & 0.00000 & -2.23529 & -2.94615 \\ C & -0.00000 & -3.57712 & -1.23947 \\ \text { C } & -0.00000 & -3.66456 & 0.16395 \\ \text { C } & -0.00000 & -2.51221 & 0.92964 \\ H & -0.00000 & 4.48410 & -1.84565 \\ H & 0.00000 & 2.23529 & -2.94615 \\ H & -0.00000 & 2.54794 & 2.02034 \\ H & -0.00000 & 4.64125 & 0.64897 \\ H & -0.00000 & -4.48410 & -1.84565 \\ H & -0.00000 & -4.64125 & 0.64897 \\ H & -0.00000 & -2.54794 & 2.02034 \\ \text { S } & 0.00000 & 0.00000 & 2.78754 \\ \text { O } & -0.00000 & -0.00000 & -1.76727\end{array}$

\subsubsection{SS}

$\begin{array}{lrrr}23 & & & \\ \text { SS @ PBE-D3/def2-TZVP } & & \\ \text { C } & 0.49651 & -0.26315 & 3.70834 \\ \text { C } & 1.13341 & -0.20036 & 2.48290 \\ \text { C } & 0.42437 & -0.03401 & 1.26934 \\ \text { C } & -0.98713 & 0.04744 & 1.36341 \\ \text { C } & -1.63517 & 0.00249 & 2.61429 \\ \text { C } & -0.90179 & -0.15001 & 3.77796 \\ \text { C } & 1.16432 & 0.06205 & -0.00000 \\ \text { C } & -0.98713 & 0.04744 & -1.36341 \\ \text { C } & 0.42437 & -0.03401 & -1.26934 \\ \text { C } & 1.13341 & -0.20036 & -2.48290 \\ \text { H } & 2.21928 & -0.27055 & -2.40834 \\ \text { C } & 0.49651 & -0.26315 & -3.70834 \\ \text { C } & -0.90179 & -0.15001 & -3.77796 \\ \text { C } & -1.63517 & 0.00249 & -2.61429 \\ \text { H } & 1.08175 & -0.40021 & 4.61835 \\ \text { H } & 2.21928 & -0.27055 & 2.40834 \\ \text { H } & -2.72366 & 0.07896 & 2.65196 \\ \text { H } & -1.41432 & -0.19114 & 4.74015 \\ \text { H } & 1.08175 & -0.40021 & -4.61835 \\ \text { H } & -1.41432 & -0.19114 & -4.74015 \\ \text { H } & -2.72366 & 0.07896 & -2.65196 \\ \text { S } & 2.81835 & 0.28393 & -0.00000 \\ \text { S } & -2.04800 & 0.23886 & 0.00000\end{array}$




\subsubsection{FC}

\begin{tabular}{|c|c|c|c|}
\hline \multicolumn{4}{|c|}{ Fc@PBE-D3/def2-TZVP } \\
\hline $\mathrm{C}$ & -0.30245 & 0.28127 & 1.32199 \\
\hline$S$ & 0.01220 & 0.92437 & 2.81562 \\
\hline $\mathrm{C}$ & -1.44274 & 0.79948 & 0.51964 \\
\hline $\mathrm{C}$ & -2.52961 & -0.05079 & 0.13119 \\
\hline $\mathrm{C}$ & -1.45105 & 2.13396 & 0.14182 \\
\hline $\mathrm{C}$ & -2.64394 & -1.39919 & 0.56323 \\
\hline $\mathrm{C}$ & -3.57781 & 0.49559 & -0.68664 \\
\hline $\mathrm{C}$ & -2.47597 & 2.66046 & -0.66976 \\
\hline $\mathrm{H}$ & -0.63774 & 2.77695 & 0.48035 \\
\hline $\mathrm{C}$ & -3.71515 & -2.17947 & 0.18186 \\
\hline $\mathrm{H}$ & -0.35670 & -0.97046 & -1.35829 \\
\hline C & -4.66310 & -0.33849 & -1.06541 \\
\hline $\mathrm{C}$ & -3.51518 & 1.85509 & -1.08499 \\
\hline $\mathrm{H}$ & -2.43876 & 3.70949 & -0.96748 \\
\hline C & -4.73094 & -1.64938 & -0.64699 \\
\hline $\mathrm{H}$ & -3.78367 & -3.21193 & 0.52817 \\
\hline $\mathrm{H}$ & -5.45246 & 0.08661 & -1.68948 \\
\hline $\mathrm{H}$ & -4.31316 & 2.25499 & -1.71435 \\
\hline $\mathrm{H}$ & -5.57282 & -2.27730 & -0.94280 \\
\hline C & 0.49964 & -0.77616 & 0.72616 \\
\hline C & 0.40064 & -1.26317 & -0.63811 \\
\hline $\mathrm{C}$ & 1.66684 & -1.39431 & 1.31452 \\
\hline $\mathrm{C}$ & 1.46875 & -2.18240 & -0.85907 \\
\hline $\mathrm{H}$ & -1.87547 & -1.81510 & 1.21494 \\
\hline $\mathrm{C}$ & 2.24803 & -2.26171 & 0.34223 \\
\hline $\mathrm{H}$ & 2.01928 & -1.19945 & 2.32265 \\
\hline $\mathrm{H}$ & 1.67626 & -2.70510 & -1.78908 \\
\hline $\mathrm{H}$ & 3.14816 & -2.85545 & 0.47743 \\
\hline $\mathrm{Fe}$ & 2.17613 & -0.32852 & -0.34190 \\
\hline C & 4.10818 & 0.18749 & -0.76763 \\
\hline C & 3.58054 & 1.03874 & 0.25894 \\
\hline C & 3.28095 & 0.32980 & -1.93073 \\
\hline $\mathrm{H}$ & 4.96808 & -0.46999 & -0.67306 \\
\hline C & 2.43152 & 1.70403 & -0.27165 \\
\hline H & 3.96076 & 1.13672 & 1.27174 \\
\hline C & 2.24061 & 1.26752 & -1.62270 \\
\hline $\mathrm{H}$ & 3.40479 & -0.20195 & -2.87020 \\
\hline $\mathrm{H}$ & 1.77811 & 2.37087 & 0.28515 \\
\hline H & 1.43399 & 1.57271 & -2.28354 \\
\hline
\end{tabular}

\subsubsection{CO}

$\begin{array}{lrrr}10 & & \\ C O & 0.18505 \\ \mathrm{C} & -0.00000 & 0.00000 & 0.1 .40432 \\ \mathrm{O} & -0.00000 & 0.00000 & 1.0 .61468 \\ \mathrm{C} & 0.00000 & 1.28791 & -1.27219 \\ \mathrm{H} & 0.88245 & 1.32884 & -1.27219 \\ \mathrm{H} & -0.88245 & 1.32884 & 0.06003 \\ \mathrm{H} & 0.00000 & 2.15077 & -0.61468 \\ \mathrm{C} & -0.00000 & -1.28791 & -1.27219 \\ \mathrm{H} & -0.88245 & -1.32884 & -1.27219 \\ \mathrm{H} & 0.88245 & -1.32884 & 0.06003 \\ \mathrm{H} & -0.00000 & -2.15077 & \end{array}$




\subsubsection{CS}

$\begin{array}{lccc}10 & & & \\ \mathrm{CS} & & \\ \mathrm{C} & 0.00000 & -0.00000 & -0.25762 \\ \mathrm{C} & 0.00000 & 1.26701 & -1.06225 \\ \mathrm{H} & 0.88095 & 1.29341 & -1.72608 \\ \mathrm{H} & -0.88095 & 1.29341 & -1.72608 \\ \mathrm{H} & 0.00000 & 2.15397 & -0.42108 \\ \mathrm{C} & -0.00000 & -1.26701 & -1.06225 \\ \mathrm{H} & -0.88095 & -1.29341 & -1.72608 \\ \mathrm{H} & 0.88095 & -1.29341 & -1.72608 \\ \mathrm{H} & -0.00000 & -2.15397 & -0.42108 \\ \mathrm{~S} & -0.00000 & 0.00000 & 1.37745\end{array}$

\subsection{8 $0 \mathrm{O}_{\text {dist }}$}

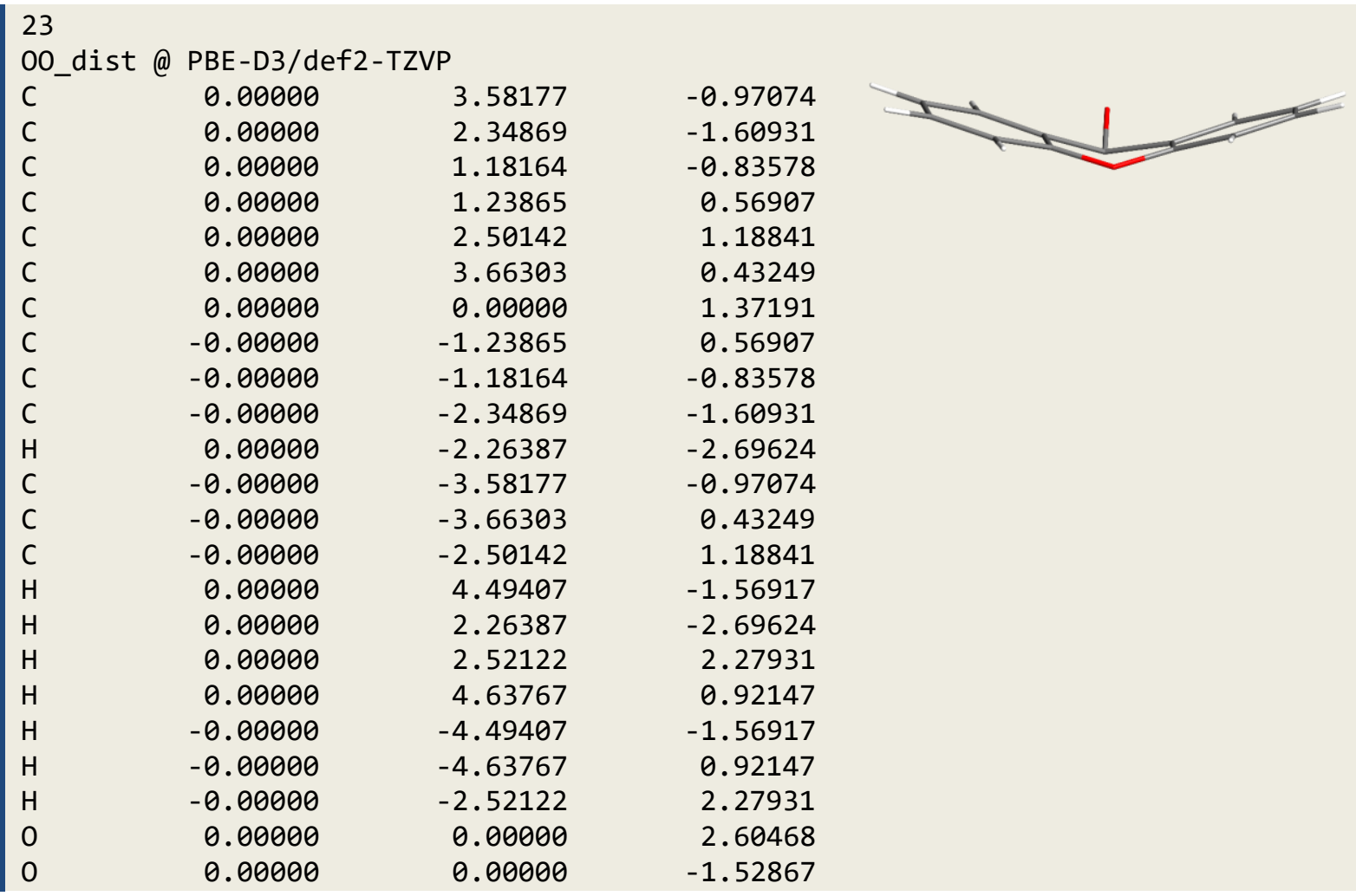

\subsection{9 $\quad \mathrm{OS}_{\mathrm{dist}}$}

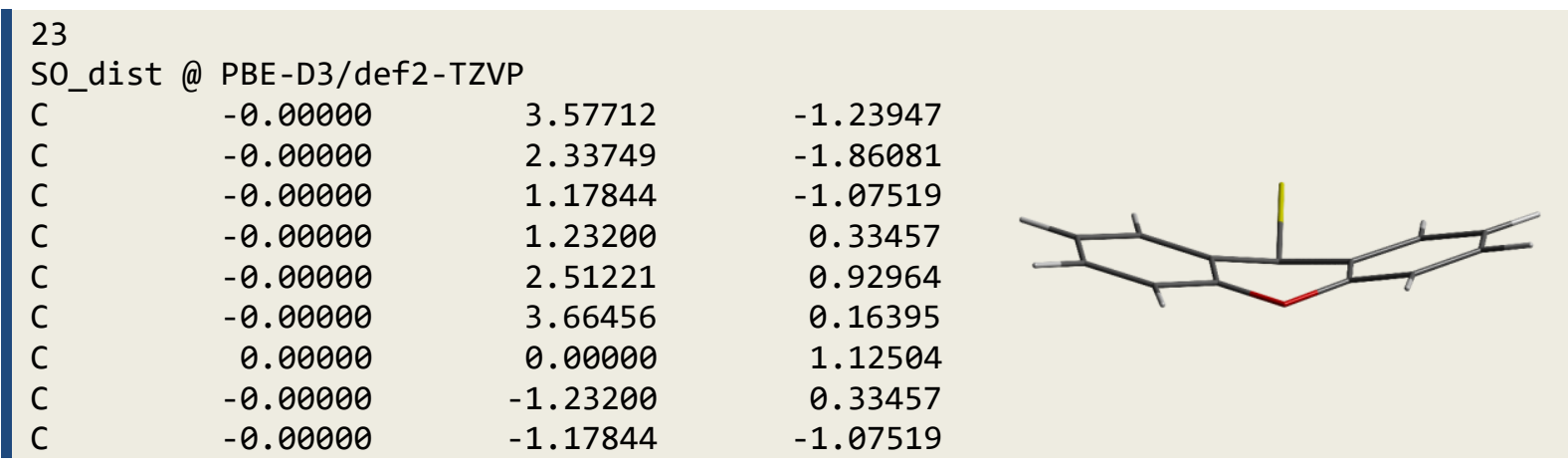




$\begin{array}{lrrr}C & -0.00000 & -2.33749 & -1.86081 \\ H & 0.00000 & -2.23529 & -2.94615 \\ C & -0.00000 & -3.57712 & -1.23947 \\ C & -0.00000 & -3.66456 & 0.16395 \\ C & -0.00000 & -2.51221 & 0.92964 \\ H & -0.00000 & 4.48410 & -1.84565 \\ H & 0.00000 & 2.23529 & -2.94615 \\ H & -0.00000 & 2.54794 & 2.02034 \\ H & -0.00000 & 4.64125 & 0.64897 \\ H & -0.00000 & -4.48410 & -1.84565 \\ H & -0.00000 & -4.64125 & 0.64897 \\ H & -0.00000 & -2.54794 & 2.02034 \\ \text { S } & 0.00000 & 0.00000 & 2.78754 \\ O & -0.00000 & -0.00000 & -1.76727\end{array}$

\subsubsection{0 $\mathrm{SS}_{\text {dist }}$}

\begin{tabular}{lrrr}
23 & \multicolumn{3}{l}{} \\
SS_dist @ PBE-D3/def2-TZVP & \\
C & 0.49651 & -0.26315 & 3.70834 \\
$\mathrm{C}$ & 1.13341 & -0.20036 & 2.48290 \\
$\mathrm{C}$ & 0.42437 & -0.03401 & 1.26934 \\
$\mathrm{C}$ & -0.98713 & 0.04744 & 1.36341 \\
$\mathrm{C}$ & -1.63517 & 0.00249 & 2.61429 \\
$\mathrm{C}$ & -0.90179 & -0.15001 & 3.77796 \\
$\mathrm{C}$ & 1.16432 & 0.06205 & -0.00000 \\
$\mathrm{C}$ & -0.98713 & 0.04744 & -1.36341 \\
$\mathrm{C}$ & 0.42437 & -0.03401 & -1.26934 \\
$\mathrm{C}$ & 1.13341 & -0.20036 & -2.48290 \\
$\mathrm{H}$ & 2.21928 & -0.27055 & -2.40834 \\
$\mathrm{C}$ & 0.49651 & -0.26315 & -3.70834 \\
$\mathrm{C}$ & -0.90179 & -0.15001 & -3.77796 \\
$\mathrm{C}$ & -1.63517 & 0.00249 & -2.61429 \\
$\mathrm{H}$ & 1.08175 & -0.40021 & 4.61835 \\
$\mathrm{H}$ & 2.21928 & -0.27055 & 2.40834 \\
$\mathrm{H}$ & -2.72366 & 0.07896 & 2.65196 \\
$\mathrm{H}$ & -1.41432 & -0.19114 & 4.74015 \\
$\mathrm{H}$ & 1.08175 & -0.40021 & -4.61835 \\
$\mathrm{H}$ & -1.41432 & -0.19114 & -4.74015 \\
$\mathrm{H}$ & -2.72366 & 0.07896 & -2.65196 \\
$\mathrm{~S}$ & 2.81835 & 0.28393 & -0.00000 \\
$\mathrm{~S}$ & -2.04800 & 0.23886 & 0.00000 \\
& & &
\end{tabular}

\subsubsection{1 $\quad \mathrm{FC}_{\text {dist }}$}

39
FC_dist @




\begin{tabular}{|c|c|c|c|}
\hline $\mathrm{H}$ & -0.35670 & -0.97046 & -1.35829 \\
\hline C & -4.66310 & -0.33849 & -1.06541 \\
\hline C & -3.51518 & 1.85509 & -1.08499 \\
\hline $\mathrm{H}$ & -2.43876 & 3.70949 & -0.96748 \\
\hline C & -4.73094 & -1.64938 & -0.64699 \\
\hline $\mathrm{H}$ & -3.78367 & -3.21193 & 0.52817 \\
\hline $\mathrm{H}$ & -5.45246 & 0.08661 & -1.68948 \\
\hline $\mathrm{H}$ & -4.31316 & 2.25499 & -1.71435 \\
\hline $\mathrm{H}$ & -5.57282 & -2.27730 & -0.94280 \\
\hline C & 0.49964 & -0.77616 & 0.72616 \\
\hline C & 0.40064 & -1.26317 & -0.63811 \\
\hline C & 1.66684 & -1.39431 & 1.31452 \\
\hline C & 1.46875 & -2.18240 & -0.85907 \\
\hline $\mathrm{H}$ & -1.87547 & -1.81510 & 1.21494 \\
\hline C & 2.24803 & -2.26171 & 0.34223 \\
\hline $\mathrm{H}$ & 2.01928 & -1.19945 & 2.32265 \\
\hline $\mathrm{H}$ & 1.67626 & -2.70510 & -1.78908 \\
\hline $\mathrm{H}$ & 3.14816 & -2.85545 & 0.47743 \\
\hline $\mathrm{Fe}$ & 2.17613 & -0.32852 & -0.34190 \\
\hline $\mathrm{C}$ & 4.10818 & 0.18749 & -0.76763 \\
\hline C & 3.58054 & 1.03874 & 0.25894 \\
\hline C & 3.28095 & 0.32980 & -1.93073 \\
\hline $\mathrm{H}$ & 4.96808 & -0.46999 & -0.67306 \\
\hline C & 2.43152 & 1.70403 & -0.27165 \\
\hline $\mathrm{H}$ & 3.96076 & 1.13672 & 1.27174 \\
\hline C & 2.24061 & 1.26752 & -1.62270 \\
\hline $\mathrm{H}$ & 3.40479 & -0.20195 & -2.87020 \\
\hline $\mathrm{H}$ & 1.77811 & 2.37087 & 0.28515 \\
\hline $\mathrm{H}$ & 1.43399 & 1.57271 & -2.28354 \\
\hline
\end{tabular}

\subsubsection{2 $\mathrm{CO}_{\text {dist }}$}

$\begin{array}{lcrr}10 & & \\ \mathrm{CO}_{\text {dist }} @ \mathrm{PBE}-\mathrm{D} 3 / \mathrm{def}-\mathrm{TZVP} & & \\ \mathrm{C} & -0.00150 & 0.08798 & -0.41935 \\ \mathrm{O} & 0.13015 & 1.45454 & 0.08957 \\ \mathrm{C} & -1.30839 & -0.52715 & 0.05804 \\ \mathrm{H} & -1.40695 & -1.54957 & -0.33403 \\ \mathrm{H} & -1.32011 & -0.58414 & 1.15722 \\ \mathrm{H} & -2.18045 & 0.05395 & -0.26606 \\ \mathrm{C} & 1.20209 & -0.70279 & 0.05626 \\ \mathrm{H} & 1.19012 & -0.76794 & 1.15560 \\ \mathrm{H} & 1.18684 & -1.72198 & -0.35258 \\ \mathrm{H} & 2.13620 & -0.21488 & -0.24646\end{array}$

\subsubsection{3 $\mathrm{CS}_{\mathrm{dist}}$}

$\begin{array}{lcrr}10 & & & \\ \text { CS } \text { dist @ PBE-D3/def2-TZVP } & & -0.44944 \\ \mathrm{C} & 0.37091 & 0.00142 & 0.06836 \\ \mathrm{C} & 1.10905 & -1.23528 & -0.24524 \\ \mathrm{H} & 2.16473 & -1.20344 & 1.16844 \\ \mathrm{H} & 1.09006 & -1.26149 & -0.30149 \\ \mathrm{H} & 0.66366 & -2.16778 & 0.06758 \\ \mathrm{C} & 1.02352 & 1.27977 & 1.16818 \\ \mathrm{H} & 1.04614 & 1.27997 & -0.29432 \\ \mathrm{H} & 2.05996 & 1.35668 & \end{array}$




$\begin{array}{rrrr}\mathrm{H} & 0.46875 & 2.16638 & -0.25903 \\ \mathrm{~S} & -1.40714 & -0.02786 & 0.04028\end{array}$

\subsubsection{4 $[\mathrm{P}(\mu-\mathrm{NTer})]_{2}$}

\begin{tabular}{|c|c|c|c|}
\hline \multicolumn{4}{|c|}{$\begin{array}{l}102 \\
{[\mathrm{P}(\mu \text {-NTer })]_{2} @ \mathrm{PBE}-\mathrm{D} 3 / \text { def2-TZVP }}\end{array}$} \\
\hline $\mathrm{N}$ & -0.00000 & 0.00000 & -1.11373 \\
\hline $\mathrm{N}$ & -0.00000 & 0.00000 & 1.11373 \\
\hline$P$ & 1.34195 & -0.00000 & 0.00000 \\
\hline$P$ & -1.34195 & 0.00000 & -0.00000 \\
\hline$C$ & 0.00000 & 0.00000 & 2.51579 \\
\hline$C$ & 1.15163 & 0.41324 & 3.22973 \\
\hline$C$ & -1.15163 & -0.41324 & 3.22973 \\
\hline$C$ & 1.13077 & 0.40398 & 4.62874 \\
\hline$C$ & -1.13077 & -0.40398 & 4.62874 \\
\hline$C$ & 0.00000 & 0.00000 & 5.33560 \\
\hline $\mathrm{H}$ & 2.02606 & 0.73377 & 5.15966 \\
\hline $\mathrm{H}$ & -2.02606 & -0.73377 & 5.15966 \\
\hline $\mathrm{H}$ & 0.00000 & 0.00000 & 6.42632 \\
\hline $\mathrm{C}$ & 0.00000 & -0.00000 & -2.51579 \\
\hline $\mathrm{C}$ & 1.15163 & -0.41324 & -3.22973 \\
\hline $\mathrm{C}$ & -1.15163 & 0.41324 & -3.22973 \\
\hline $\mathrm{C}$ & 1.13077 & -0.40398 & -4.62874 \\
\hline $\mathrm{C}$ & -1.13077 & 0.40398 & -4.62874 \\
\hline $\mathrm{C}$ & 0.00000 & -0.00000 & -5.33560 \\
\hline $\mathrm{H}$ & 2.02606 & -0.73377 & -5.15966 \\
\hline $\mathrm{H}$ & -2.02606 & 0.73377 & -5.15966 \\
\hline $\mathrm{H}$ & 0.00000 & -0.00000 & -6.42632 \\
\hline$C$ & -2.38046 & 0.88753 & -2.53313 \\
\hline $\mathrm{C}$ & -3.49608 & 0.03545 & -2.39727 \\
\hline $\mathrm{C}$ & -2.43121 & 2.20977 & -2.03338 \\
\hline $\mathrm{C}$ & -4.64223 & 0.52091 & -1.75802 \\
\hline $\mathrm{C}$ & -3.59630 & 2.65199 & -1.40489 \\
\hline $\mathrm{C}$ & -4.71163 & 1.82010 & -1.25104 \\
\hline $\mathrm{H}$ & -5.50022 & -0.14529 & -1.63830 \\
\hline $\mathrm{H}$ & -3.62927 & 3.67225 & -1.01241 \\
\hline $\mathrm{C}$ & 2.38046 & -0.88753 & -2.53313 \\
\hline $\mathrm{C}$ & 3.49608 & -0.03545 & -2.39727 \\
\hline $\mathrm{C}$ & 2.43121 & -2.20977 & -2.03338 \\
\hline $\mathrm{C}$ & 4.64223 & -0.52091 & -1.75802 \\
\hline $\mathrm{C}$ & 3.59630 & -2.65199 & -1.40489 \\
\hline $\mathrm{C}$ & 4.71163 & -1.82010 & -1.25104 \\
\hline $\mathrm{H}$ & 5.50022 & 0.14529 & -1.63830 \\
\hline $\mathrm{H}$ & 3.62927 & -3.67225 & -1.01241 \\
\hline $\mathrm{C}$ & -2.38046 & -0.88753 & 2.53313 \\
\hline $\mathrm{C}$ & -3.49608 & -0.03545 & 2.39727 \\
\hline $\mathrm{C}$ & -2.43121 & -2.20977 & 2.03338 \\
\hline $\mathrm{C}$ & -4.64223 & -0.52091 & 1.75802 \\
\hline $\mathrm{C}$ & -3.59630 & -2.65199 & 1.40489 \\
\hline $\mathrm{C}$ & -4.71163 & -1.82010 & 1.25104 \\
\hline $\mathrm{H}$ & -5.50022 & 0.14529 & 1.63830 \\
\hline $\mathrm{H}$ & -3.62927 & -3.67225 & 1.01241 \\
\hline $\mathrm{C}$ & 2.38046 & 0.88753 & 2.53313 \\
\hline $\mathrm{C}$ & 2.43121 & 2.20977 & 2.03338 \\
\hline $\mathrm{C}$ & 3.49608 & 0.03545 & 2.39727 \\
\hline $\mathrm{C}$ & 3.59630 & 2.65199 & 1.40489 \\
\hline $\mathrm{C}$ & 4.64223 & 0.52091 & 1.75802 \\
\hline
\end{tabular}




\begin{tabular}{|c|c|c|c|}
\hline $\mathrm{C}$ & 4.71163 & 1.82010 & 1.25104 \\
\hline $\mathrm{H}$ & 3.62927 & 3.67225 & 1.01241 \\
\hline $\mathrm{H}$ & 5.50022 & -0.14529 & 1.63830 \\
\hline$C$ & -3.44308 & 1.39457 & 2.86433 \\
\hline $\mathrm{H}$ & -4.42259 & 1.87761 & 2.75186 \\
\hline $\mathrm{H}$ & -3.13262 & 1.47614 & 3.91534 \\
\hline $\mathrm{H}$ & -2.71205 & 1.96531 & 2.26892 \\
\hline$C$ & -1.23307 & -3.11288 & 2.13905 \\
\hline $\mathrm{H}$ & -0.84811 & -3.15778 & 3.16829 \\
\hline $\mathrm{H}$ & -1.47899 & -4.13121 & 1.81133 \\
\hline $\mathrm{H}$ & -0.40953 & -2.74129 & 1.50731 \\
\hline$C$ & -5.94790 & -2.31722 & 0.55158 \\
\hline $\mathrm{H}$ & -5.71637 & -2.65277 & -0.47111 \\
\hline $\mathrm{H}$ & -6.38856 & -3.17687 & 1.07961 \\
\hline $\mathrm{H}$ & -6.71366 & -1.53262 & 0.48676 \\
\hline$C$ & 3.44308 & -1.39457 & 2.86433 \\
\hline $\mathrm{H}$ & 4.42259 & -1.87761 & 2.75186 \\
\hline $\mathrm{H}$ & 3.13262 & -1.47614 & 3.91534 \\
\hline $\mathrm{H}$ & 2.71205 & -1.96531 & 2.26892 \\
\hline$C$ & 1.23307 & 3.11288 & 2.13905 \\
\hline $\mathrm{H}$ & 0.40953 & 2.74129 & 1.50731 \\
\hline $\mathrm{H}$ & 0.84811 & 3.15778 & 3.16829 \\
\hline $\mathrm{H}$ & 1.47899 & 4.13121 & 1.81133 \\
\hline$C$ & 5.94790 & 2.31722 & 0.55158 \\
\hline $\mathrm{H}$ & 5.71637 & 2.65277 & -0.47111 \\
\hline $\mathrm{H}$ & 6.38856 & 3.17687 & 1.07961 \\
\hline $\mathrm{H}$ & 6.71366 & 1.53262 & 0.48676 \\
\hline$C$ & 5.94790 & -2.31722 & -0.55158 \\
\hline $\mathrm{H}$ & 5.71637 & -2.65277 & 0.47111 \\
\hline $\mathrm{H}$ & 6.38856 & -3.17687 & -1.07961 \\
\hline $\mathrm{H}$ & 6.71366 & -1.53262 & -0.48676 \\
\hline$C$ & -5.94790 & 2.31722 & -0.55158 \\
\hline $\mathrm{H}$ & -5.71637 & 2.65277 & 0.47111 \\
\hline $\mathrm{H}$ & -6.38856 & 3.17687 & -1.07961 \\
\hline $\mathrm{H}$ & -6.71366 & 1.53262 & -0.48676 \\
\hline $\mathrm{C}$ & -1.23307 & 3.11288 & -2.13905 \\
\hline $\mathrm{H}$ & -0.84811 & 3.15778 & -3.16829 \\
\hline $\mathrm{H}$ & -1.47899 & 4.13121 & -1.81133 \\
\hline $\mathrm{H}$ & -0.40953 & 2.74129 & -1.50731 \\
\hline$C$ & 1.23307 & -3.11288 & -2.13905 \\
\hline $\mathrm{H}$ & 0.84811 & -3.15778 & -3.16829 \\
\hline $\mathrm{H}$ & 1.47899 & -4.13121 & -1.81133 \\
\hline $\mathrm{H}$ & 0.40953 & -2.74129 & -1.50731 \\
\hline $\mathrm{C}$ & 3.44308 & 1.39457 & -2.86433 \\
\hline $\mathrm{H}$ & 4.42259 & 1.87761 & -2.75186 \\
\hline $\mathrm{H}$ & 3.13262 & 1.47614 & -3.91534 \\
\hline $\mathrm{H}$ & 2.71205 & 1.96531 & -2.26892 \\
\hline $\mathrm{C}$ & -3.44308 & -1.39457 & -2.86433 \\
\hline $\mathrm{H}$ & -4.42259 & -1.87761 & -2.75186 \\
\hline $\mathrm{H}$ & -3.13262 & -1.47614 & -3.91534 \\
\hline $\mathrm{H}$ & -2.71205 & -1.96531 & -2.26892 \\
\hline
\end{tabular}




\subsubsection{5 $[\mathrm{P}(\mu-\mathrm{NTer})]_{2 \mathrm{dist}}(\mathrm{SO})$}

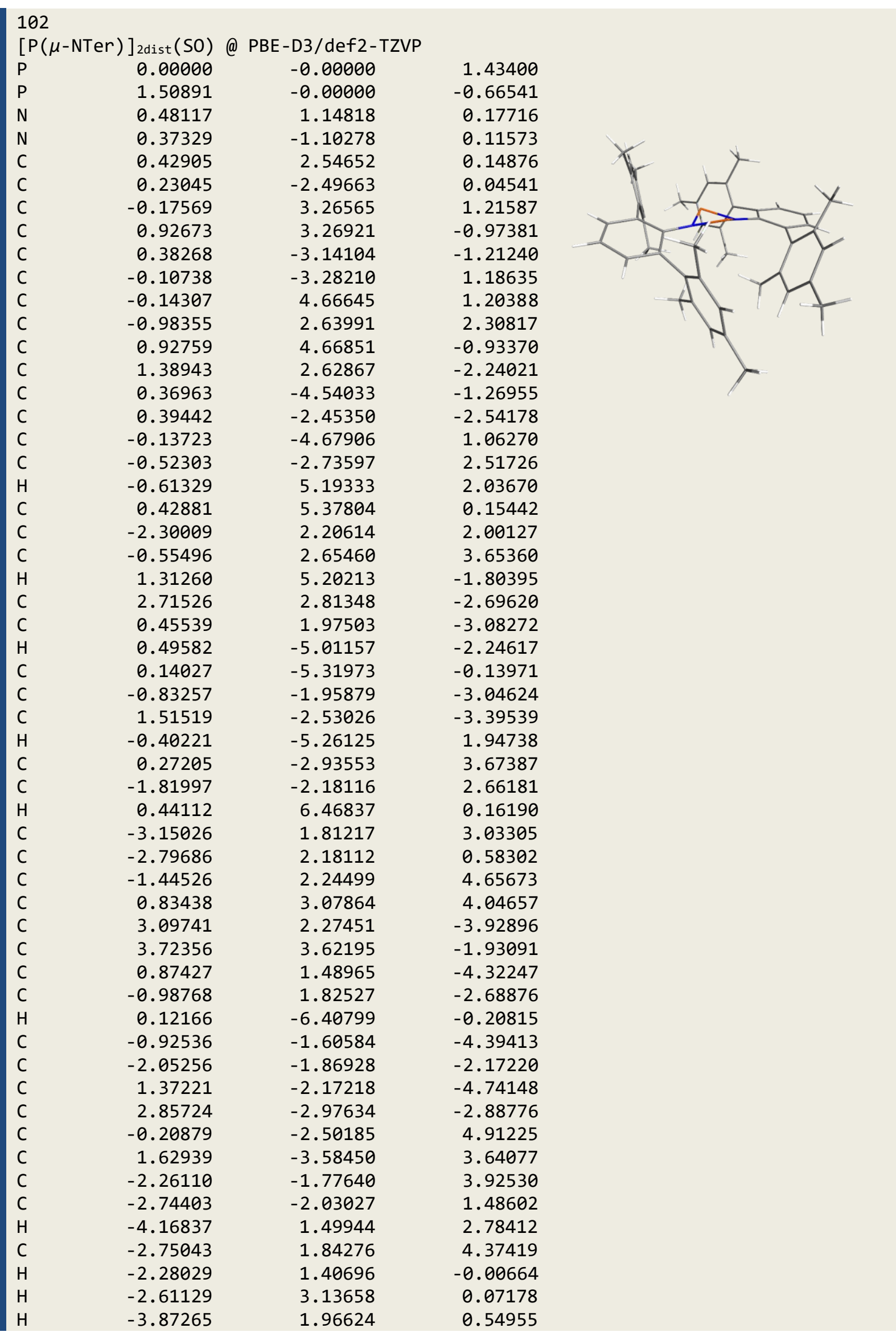




\begin{tabular}{|c|c|c|c|}
\hline $\mathrm{H}$ & -1.10912 & 2.27386 & 5.69706 \\
\hline $\mathrm{H}$ & 0.85722 & 3.42134 & 5.08968 \\
\hline $\mathrm{H}$ & 1.21795 & 3.88319 & 3.40628 \\
\hline $\mathrm{H}$ & 1.53245 & 2.22958 & 3.95943 \\
\hline $\mathrm{H}$ & 4.13218 & 2.40628 & -4.25778 \\
\hline$C$ & 2.19690 & 1.60932 & -4.76035 \\
\hline $\mathrm{H}$ & 3.54612 & 3.59843 & -0.85194 \\
\hline $\mathrm{H}$ & 3.69022 & 4.68007 & -2.23872 \\
\hline $\mathrm{H}$ & 4.74038 & 3.25967 & -2.12752 \\
\hline $\mathrm{H}$ & 0.13881 & 1.00263 & -4.96439 \\
\hline $\mathrm{H}$ & -1.11132 & 1.04999 & -1.91747 \\
\hline $\mathrm{H}$ & -1.59657 & 1.53127 & -3.55305 \\
\hline $\mathrm{H}$ & -1.39093 & 2.75845 & -2.27080 \\
\hline $\mathrm{H}$ & -1.88678 & -1.25135 & -4.77735 \\
\hline$C$ & 0.15758 & -1.73363 & -5.27013 \\
\hline $\mathrm{H}$ & -2.30070 & -2.84548 & -1.72920 \\
\hline $\mathrm{H}$ & -2.91988 & -1.51754 & -2.74560 \\
\hline $\mathrm{H}$ & -1.89102 & -1.17622 & -1.33366 \\
\hline $\mathrm{H}$ & 2.24023 & -2.26307 & -5.40028 \\
\hline $\mathrm{H}$ & 2.80415 & -3.92143 & -2.33210 \\
\hline $\mathrm{H}$ & 3.27516 & -2.23334 & -2.19088 \\
\hline $\mathrm{H}$ & 3.56966 & -3.09646 & -3.71431 \\
\hline $\mathrm{H}$ & 0.41839 & -2.65166 & 5.79556 \\
\hline$C$ & -1.46511 & -1.91081 & 5.06393 \\
\hline $\mathrm{H}$ & 1.82173 & -4.11360 & 4.58406 \\
\hline $\mathrm{H}$ & 2.41439 & -2.82136 & 3.52183 \\
\hline $\mathrm{H}$ & 1.73711 & -4.29883 & 2.81643 \\
\hline $\mathrm{H}$ & -3.26352 & -1.35143 & 4.01653 \\
\hline $\mathrm{H}$ & -2.70297 & -2.90125 & 0.81851 \\
\hline $\mathrm{H}$ & -2.47444 & -1.14717 & 0.88179 \\
\hline $\mathrm{H}$ & -3.77935 & -1.88851 & 1.82224 \\
\hline$C$ & -3.72595 & 1.50715 & 5.46882 \\
\hline$C$ & 2.63973 & 1.04736 & -6.08267 \\
\hline$C$ & 0.00365 & -1.42009 & -6.73397 \\
\hline$C$ & -1.93304 & -1.44688 & 6.41533 \\
\hline $\mathrm{H}$ & -4.21818 & 0.53979 & 5.28969 \\
\hline $\mathrm{H}$ & -4.52217 & 2.26588 & 5.52625 \\
\hline $\mathrm{H}$ & -3.23472 & 1.46269 & 6.44928 \\
\hline $\mathrm{H}$ & 2.90685 & -0.01650 & -5.98341 \\
\hline $\mathrm{H}$ & 3.52294 & 1.57674 & -6.46573 \\
\hline $\mathrm{H}$ & 1.84261 & 1.11230 & -6.83598 \\
\hline $\mathrm{H}$ & 0.95496 & -1.53128 & -7.27043 \\
\hline $\mathrm{H}$ & -0.35508 & -0.39103 & -6.89287 \\
\hline $\mathrm{H}$ & -0.73145 & -2.09024 & -7.20590 \\
\hline $\mathrm{H}$ & -1.53715 & -2.08695 & 7.21610 \\
\hline $\mathrm{H}$ & -3.02894 & -1.44205 & 6.48522 \\
\hline $\mathrm{H}$ & -1.58586 & -0.42053 & 6.61453 \\
\hline
\end{tabular}




\subsubsection{6 $[\mathrm{P}(\mu \text {-NTer })]_{2 \text { dist }}(\mathrm{SS})$}

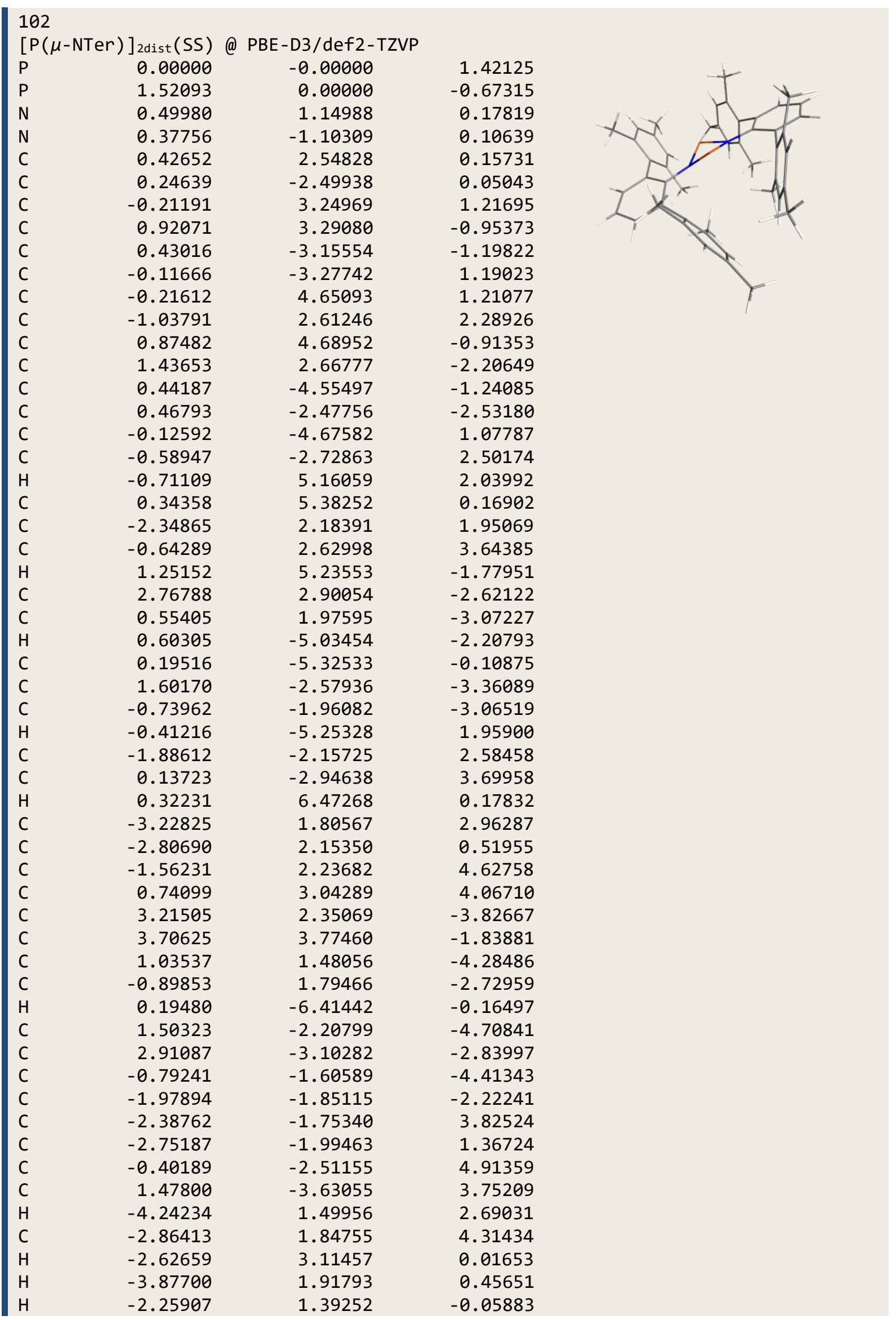




\begin{tabular}{|c|c|c|c|}
\hline $\mathrm{H}$ & -1.25321 & 2.27170 & 5.67616 \\
\hline $\mathrm{H}$ & 1.17622 & 3.78402 & 3.38506 \\
\hline $\mathrm{H}$ & 1.41314 & 2.16862 & 4.07682 \\
\hline $\mathrm{H}$ & 0.73167 & 3.46311 & 5.08192 \\
\hline $\mathrm{H}$ & 4.25619 & 2.51092 & -4.12032 \\
\hline$C$ & 2.37083 & 1.63040 & -4.67160 \\
\hline $\mathrm{H}$ & 4.74906 & 3.51186 & -2.05369 \\
\hline $\mathrm{H}$ & 3.54150 & 3.70520 & -0.75855 \\
\hline $\mathrm{H}$ & 3.57458 & 4.83352 & -2.11485 \\
\hline $\mathrm{H}$ & 0.34174 & 0.95650 & -4.94353 \\
\hline $\mathrm{H}$ & -1.33920 & 2.72308 & -2.33957 \\
\hline $\mathrm{H}$ & -1.03287 & 1.02656 & -1.95289 \\
\hline $\mathrm{H}$ & -1.46751 & 1.47443 & -3.61146 \\
\hline $\mathrm{H}$ & 2.38749 & -2.30753 & -5.34456 \\
\hline$C$ & 0.31204 & -1.74435 & -5.26346 \\
\hline $\mathrm{H}$ & 3.74727 & -2.50200 & -3.22444 \\
\hline $\mathrm{H}$ & 3.08403 & -4.14419 & -3.15646 \\
\hline $\mathrm{H}$ & 2.95063 & -3.08767 & -1.74624 \\
\hline $\mathrm{H}$ & -1.73747 & -1.23337 & -4.81965 \\
\hline $\mathrm{H}$ & -1.83034 & -1.15393 & -1.38514 \\
\hline $\mathrm{H}$ & -2.24817 & -2.82145 & -1.77900 \\
\hline $\mathrm{H}$ & -2.82804 & -1.49417 & -2.81939 \\
\hline $\mathrm{H}$ & -3.38854 & -1.31721 & 3.86572 \\
\hline$C$ & -1.65520 & -1.90413 & 5.00392 \\
\hline $\mathrm{H}$ & -2.44746 & -1.11265 & 0.77837 \\
\hline $\mathrm{H}$ & -3.80047 & -1.84460 & 1.65517 \\
\hline $\mathrm{H}$ & -2.68702 & -2.86440 & 0.70025 \\
\hline $\mathrm{H}$ & 0.17489 & -2.67818 & 5.82786 \\
\hline $\mathrm{H}$ & 1.66781 & -4.25962 & 2.87587 \\
\hline $\mathrm{H}$ & 1.54442 & -4.26321 & 4.64825 \\
\hline $\mathrm{H}$ & 2.28822 & -2.88740 & 3.81570 \\
\hline$C$ & -3.87468 & 1.53848 & 5.38473 \\
\hline$C$ & 2.88425 & 1.03831 & -5.95460 \\
\hline$C$ & 0.20209 & -1.41023 & -6.72669 \\
\hline$C$ & -2.18772 & -1.44544 & 6.33281 \\
\hline $\mathrm{H}$ & -4.66038 & 2.30983 & 5.41104 \\
\hline $\mathrm{H}$ & -3.41234 & 1.49817 & 6.37933 \\
\hline $\mathrm{H}$ & -4.37665 & 0.57676 & 5.20242 \\
\hline $\mathrm{H}$ & 2.12965 & 1.08627 & -6.75201 \\
\hline $\mathrm{H}$ & 3.14117 & -0.02374 & -5.81536 \\
\hline $\mathrm{H}$ & 3.78874 & 1.55676 & -6.30094 \\
\hline $\mathrm{H}$ & -0.14246 & -0.37576 & -6.88209 \\
\hline $\mathrm{H}$ & -0.52582 & -2.06693 & -7.22790 \\
\hline $\mathrm{H}$ & 1.16721 & -1.52398 & -7.23745 \\
\hline $\mathrm{H}$ & -1.84607 & -0.42218 & 6.55544 \\
\hline $\mathrm{H}$ & -1.83552 & -2.09257 & 7.14817 \\
\hline $\mathrm{H}$ & -3.28567 & -1.43533 & 6.34819 \\
\hline
\end{tabular}




\subsubsection{7 $[\mathrm{P}(\mu-\mathrm{NTer})]_{2 \text { dist }}(\mathrm{OO})$}

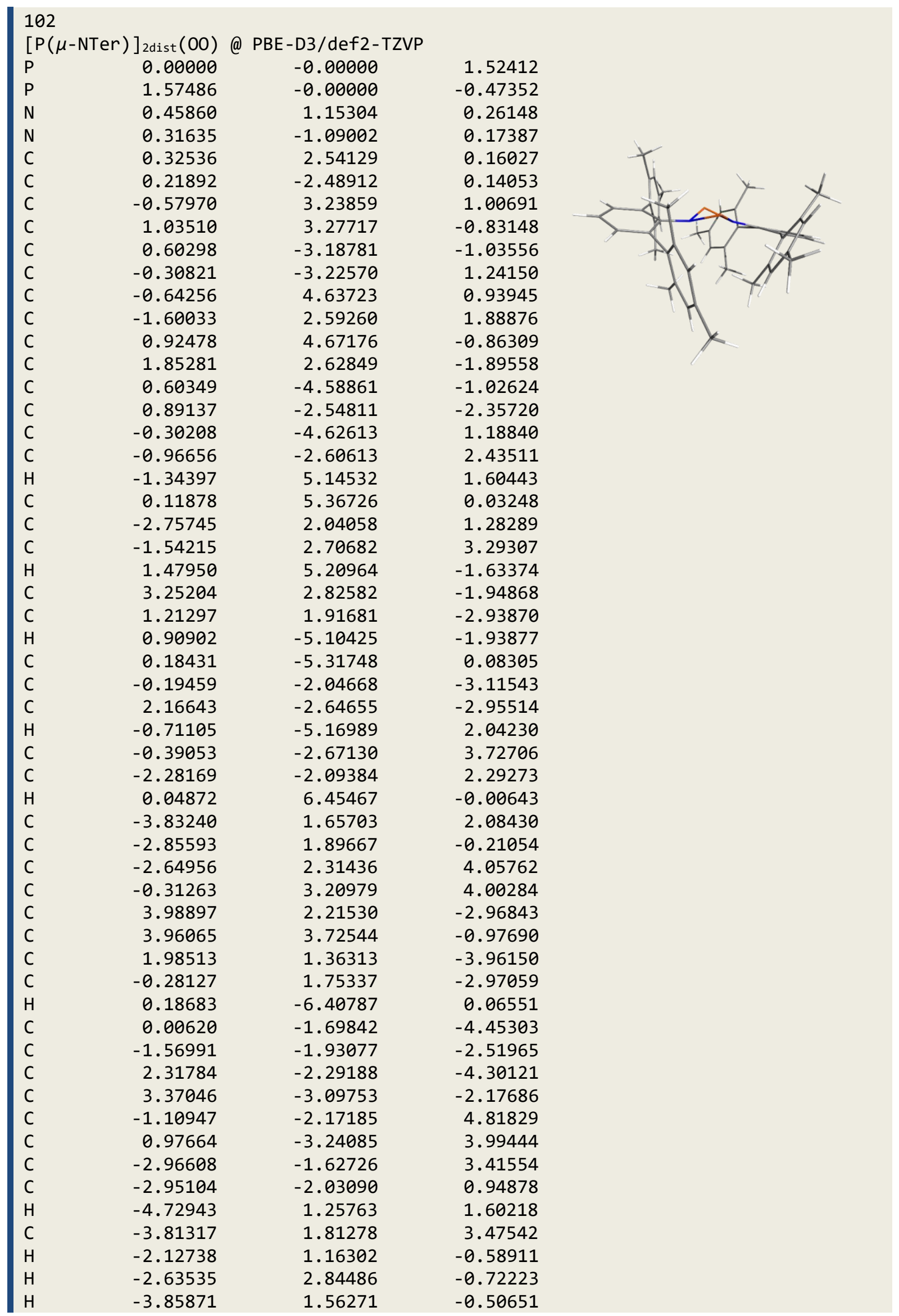




\begin{tabular}{|c|c|c|c|}
\hline $\mathrm{H}$ & -2.60425 & 2.43292 & 5.14414 \\
\hline $\mathrm{H}$ & -0.57823 & 3.71772 & 4.94009 \\
\hline $\mathrm{H}$ & 0.26919 & 3.90341 & 3.38347 \\
\hline $\mathrm{H}$ & 0.34711 & 2.36658 & 4.26802 \\
\hline $\mathrm{H}$ & 5.07451 & 2.34764 & -2.98089 \\
\hline $\mathrm{C}$ & 3.37843 & 1.47057 & -3.97985 \\
\hline $\mathrm{H}$ & 3.52056 & 3.68653 & 0.02511 \\
\hline $\mathrm{H}$ & 3.90270 & 4.77486 & -1.30924 \\
\hline $\mathrm{H}$ & 5.02306 & 3.46291 & -0.90602 \\
\hline $\mathrm{H}$ & 1.47930 & 0.81703 & -4.75836 \\
\hline $\mathrm{H}$ & -0.61561 & 1.00509 & -2.23498 \\
\hline $\mathrm{H}$ & -0.61287 & 1.41005 & -3.95857 \\
\hline $\mathrm{H}$ & -0.79646 & 2.69277 & -2.72586 \\
\hline $\mathrm{H}$ & -0.84732 & -1.33528 & -5.03302 \\
\hline $\mathrm{C}$ & 1.25111 & -1.83561 & -5.07592 \\
\hline $\mathrm{H}$ & -1.90757 & -2.88895 & -2.09770 \\
\hline $\mathrm{H}$ & -2.29780 & -1.60615 & -3.27430 \\
\hline $\mathrm{H}$ & -1.58234 & -1.20317 & -1.69453 \\
\hline $\mathrm{H}$ & 3.30517 & -2.39225 & -4.75992 \\
\hline $\mathrm{H}$ & 3.17112 & -3.98883 & -1.56946 \\
\hline $\mathrm{H}$ & 3.69513 & -2.31280 & -1.47549 \\
\hline $\mathrm{H}$ & 4.21303 & -3.31134 & -2.84758 \\
\hline $\mathrm{H}$ & -0.65097 & -2.22139 & 5.80991 \\
\hline $\mathrm{C}$ & -2.39372 & -1.64238 & 4.69021 \\
\hline $\mathrm{H}$ & 1.03212 & -3.63039 & 5.01992 \\
\hline $\mathrm{H}$ & 1.74661 & -2.46084 & 3.89483 \\
\hline $\mathrm{H}$ & 1.23708 & -4.05270 & 3.30463 \\
\hline $\mathrm{H}$ & -3.98024 & -1.24341 & 3.28645 \\
\hline $\mathrm{H}$ & -2.82150 & -2.96294 & 0.38168 \\
\hline $\mathrm{H}$ & -2.52399 & -1.22229 & 0.33360 \\
\hline $\mathrm{H}$ & -4.02407 & -1.82745 & 1.05776 \\
\hline $\mathrm{C}$ & -5.03167 & 1.49372 & 4.29833 \\
\hline $\mathrm{C}$ & 4.19882 & 0.80617 & -5.05091 \\
\hline $\mathrm{C}$ & 1.42581 & -1.50344 & -6.53353 \\
\hline $\mathrm{C}$ & -3.12718 & -1.10921 & 5.88935 \\
\hline $\mathrm{H}$ & -5.41719 & 0.48632 & 4.08049 \\
\hline $\mathrm{H}$ & -5.84649 & 2.20163 & 4.07954 \\
\hline $\mathrm{H}$ & -4.81916 & 1.55050 & 5.37373 \\
\hline $\mathrm{H}$ & 4.52491 & -0.19415 & -4.72421 \\
\hline $\mathrm{H}$ & 5.10265 & 1.38681 & -5.28297 \\
\hline $\mathrm{H}$ & 3.62226 & 0.67471 & -5.97646 \\
\hline $\mathrm{H}$ & 2.46197 & -1.66511 & -6.85888 \\
\hline $\mathrm{H}$ & 1.16603 & -0.45360 & -6.74394 \\
\hline $\mathrm{H}$ & 0.77150 & -2.12492 & -7.16395 \\
\hline $\mathrm{H}$ & -2.81767 & -1.62494 & 6.80884 \\
\hline $\mathrm{H}$ & -4.21470 & -1.21733 & 5.77980 \\
\hline $\mathrm{H}$ & -2.91645 & -0.03664 & 6.02569 \\
\hline
\end{tabular}




\subsubsection{8 $[\mathrm{P}(\mu-\mathrm{NTTer})]_{2 \text { dist }}(\mathrm{Fc})$}

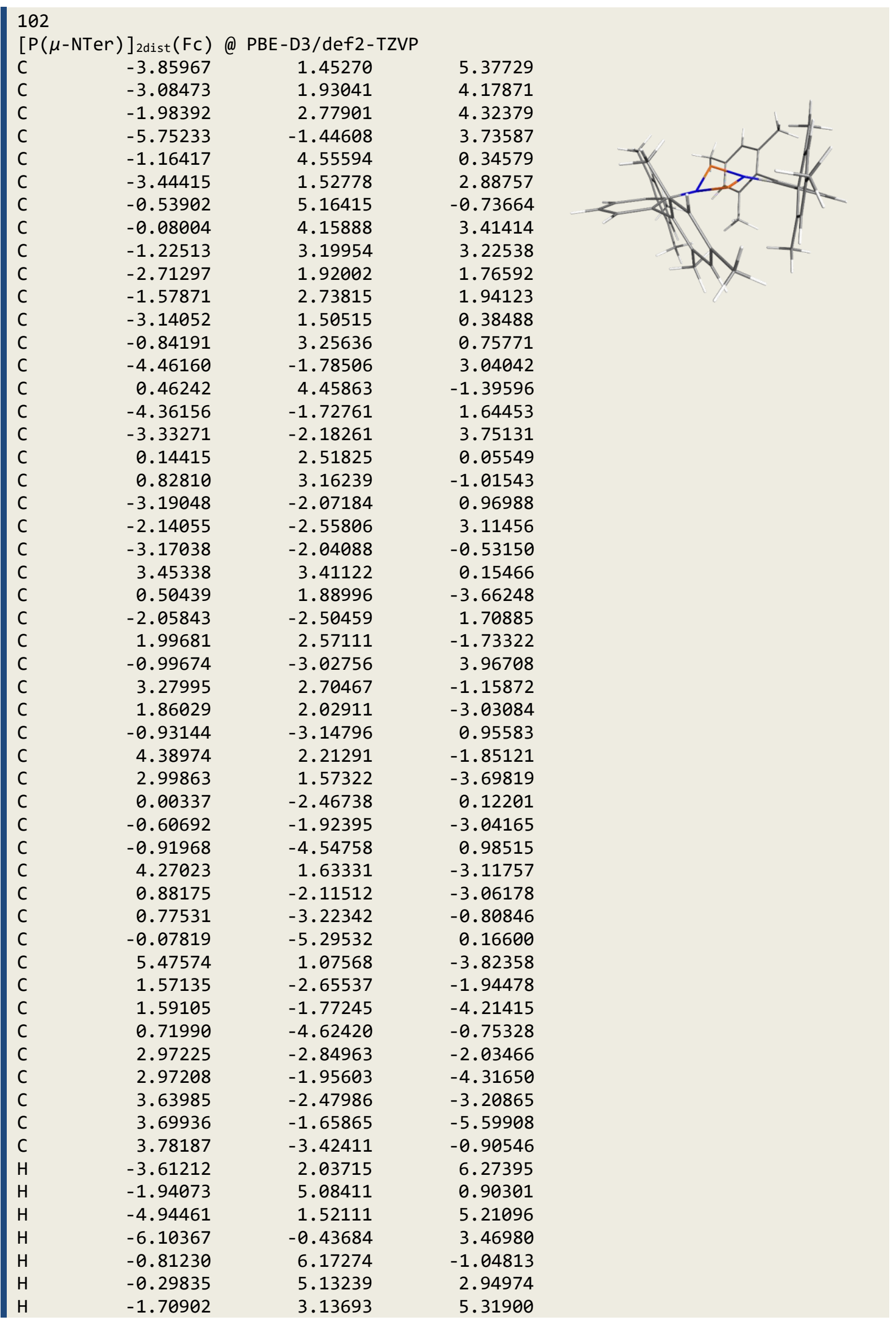




\begin{tabular}{|c|c|c|c|}
\hline $\mathrm{H}$ & -5.64155 & -1.48653 & 4.82752 \\
\hline $\mathrm{H}$ & -3.63470 & 0.39524 & 5.59060 \\
\hline $\mathrm{H}$ & -4.31504 & 0.88678 & 2.74722 \\
\hline $\mathrm{H}$ & 0.12233 & 4.32406 & 4.47927 \\
\hline $\mathrm{H}$ & -6.55174 & -2.14723 & 3.45013 \\
\hline $\mathrm{H}$ & -3.32582 & 2.38322 & -0.25222 \\
\hline $\mathrm{H}$ & -4.05111 & 0.89563 & 0.42202 \\
\hline $\mathrm{H}$ & 0.84106 & 3.78798 & 2.94139 \\
\hline $\mathrm{H}$ & -5.23410 & -1.42664 & 1.05716 \\
\hline $\mathrm{H}$ & 1.00844 & 4.91698 & -2.22336 \\
\hline $\mathrm{H}$ & -2.35817 & 0.91424 & -0.11585 \\
\hline $\mathrm{H}$ & -3.37947 & -2.23553 & 4.84323 \\
\hline $\mathrm{H}$ & -4.15243 & -1.75634 & -0.93086 \\
\hline $\mathrm{H}$ & 3.21123 & 4.48135 & 0.05571 \\
\hline $\mathrm{H}$ & -0.03064 & 2.84922 & -3.70557 \\
\hline $\mathrm{H}$ & -0.12604 & 1.20454 & -3.07119 \\
\hline $\mathrm{H}$ & -0.67599 & -2.23417 & 4.65875 \\
\hline $\mathrm{H}$ & 2.78345 & 3.00878 & 0.92514 \\
\hline $\mathrm{H}$ & -2.42866 & -1.32030 & -0.90263 \\
\hline $\mathrm{H}$ & -2.89510 & -3.02134 & -0.94776 \\
\hline $\mathrm{H}$ & 4.48653 & 3.32416 & 0.51566 \\
\hline $\mathrm{H}$ & 0.58321 & 1.48336 & -4.67892 \\
\hline $\mathrm{H}$ & -1.29343 & -3.88987 & 4.58416 \\
\hline $\mathrm{H}$ & -0.12730 & -3.31987 & 3.37303 \\
\hline $\mathrm{H}$ & -0.88999 & -1.09164 & -2.37687 \\
\hline $\mathrm{H}$ & -0.98479 & -1.69029 & -4.04516 \\
\hline $\mathrm{H}$ & -1.62900 & -5.04835 & 1.64670 \\
\hline $\mathrm{H}$ & -1.12527 & -2.81703 & -2.66707 \\
\hline $\mathrm{H}$ & 5.37865 & 2.30005 & -1.39277 \\
\hline $\mathrm{H}$ & 2.88589 & 1.14224 & -4.69536 \\
\hline $\mathrm{H}$ & 1.03849 & -1.36844 & -5.06692 \\
\hline $\mathrm{H}$ & 5.35570 & 1.10842 & -4.91502 \\
\hline $\mathrm{H}$ & 6.38793 & 1.62779 & -3.55778 \\
\hline $\mathrm{H}$ & -0.09326 & -6.38520 & 0.19936 \\
\hline $\mathrm{H}$ & 5.63508 & 0.02243 & -3.54118 \\
\hline $\mathrm{H}$ & 3.33144 & -0.73530 & -6.06933 \\
\hline $\mathrm{H}$ & 3.87518 & -2.68694 & -0.09275 \\
\hline $\mathrm{H}$ & 1.31396 & -5.18551 & -1.47710 \\
\hline $\mathrm{H}$ & 4.77993 & -1.55384 & -5.43581 \\
\hline $\mathrm{H}$ & 3.32270 & -4.32254 & -0.47256 \\
\hline $\mathrm{H}$ & 4.72104 & -2.63519 & -3.26305 \\
\hline $\mathrm{H}$ & 3.55130 & -2.47145 & -6.32811 \\
\hline $\mathrm{H}$ & 4.79144 & -3.68479 & -1.25022 \\
\hline $\mathrm{N}$ & 0.43190 & 1.17791 & 0.36261 \\
\hline $\mathrm{N}$ & 0.11069 & -1.07092 & 0.19144 \\
\hline$P$ & 0.00000 & -0.00000 & 1.59167 \\
\hline$P$ & 1.22826 & 0.00000 & -0.67417 \\
\hline
\end{tabular}




\subsubsection{9 $[\mathrm{P}(\mu-\mathrm{NTer})]_{2 \text { dist }}(\mathrm{CO})$}

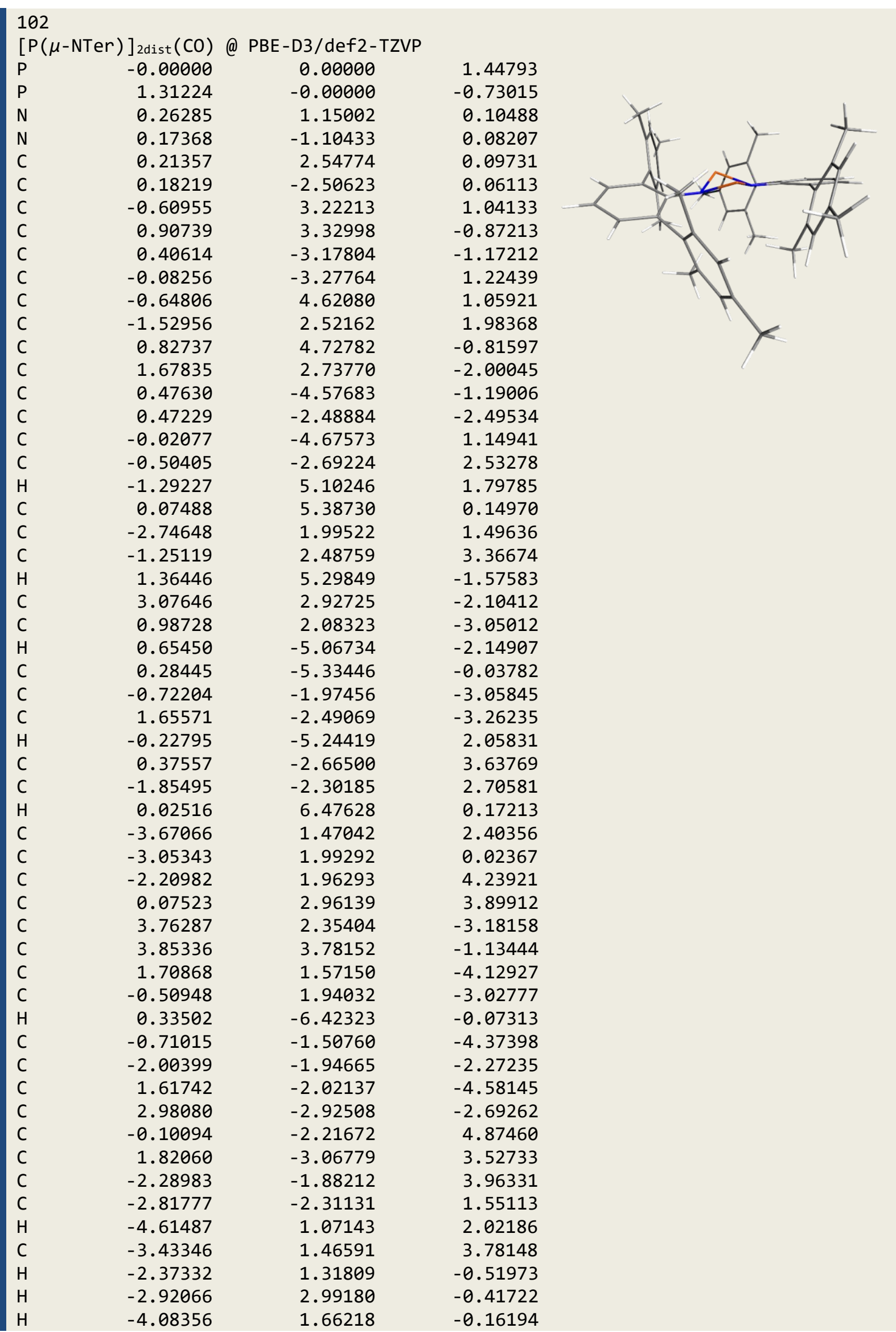




\begin{tabular}{|c|c|c|c|}
\hline $\mathrm{H}$ & -1.99269 & 1.94685 & 5.31090 \\
\hline $\mathrm{H}$ & 0.11059 & 2.87311 & 4.99282 \\
\hline $\mathrm{H}$ & 0.27981 & 4.00670 & 3.62745 \\
\hline $\mathrm{H}$ & 0.89944 & 2.35936 & 3.48340 \\
\hline $\mathrm{H}$ & 4.84832 & 2.47857 & -3.23824 \\
\hline $\mathrm{C}$ & 3.10166 & 1.66145 & -4.19829 \\
\hline $\mathrm{H}$ & 3.36346 & 3.86709 & -0.15876 \\
\hline $\mathrm{H}$ & 3.95958 & 4.80489 & -1.52951 \\
\hline $\mathrm{H}$ & 4.86862 & 3.38691 & -0.98835 \\
\hline $\mathrm{H}$ & 1.16485 & 1.07102 & -4.93142 \\
\hline $\mathrm{H}$ & -0.82188 & 1.14039 & -2.33743 \\
\hline $\mathrm{H}$ & -0.88825 & 1.67770 & -4.02354 \\
\hline $\mathrm{H}$ & -0.99880 & 2.86427 & -2.68924 \\
\hline $\mathrm{H}$ & -1.64098 & -1.12549 & -4.80291 \\
\hline $\mathrm{C}$ & 0.44556 & -1.53939 & -5.16283 \\
\hline $\mathrm{H}$ & -2.22763 & -2.92903 & -1.83119 \\
\hline $\mathrm{H}$ & -2.84701 & -1.64928 & -2.90928 \\
\hline $\mathrm{H}$ & -1.93484 & -1.23489 & -1.43531 \\
\hline $\mathrm{H}$ & 2.53861 & -2.03502 & -5.17093 \\
\hline $\mathrm{H}$ & 2.86974 & -3.50562 & -1.76995 \\
\hline $\mathrm{H}$ & 3.59326 & -2.03844 & -2.45812 \\
\hline $\mathrm{H}$ & 3.54757 & -3.52736 & -3.41696 \\
\hline $\mathrm{H}$ & 0.58803 & -2.19322 & 5.72380 \\
\hline $\mathrm{C}$ & -1.42634 & -1.82222 & 5.06168 \\
\hline $\mathrm{H}$ & 2.16199 & -3.55030 & 4.45370 \\
\hline $\mathrm{H}$ & 2.44209 & -2.17437 & 3.36620 \\
\hline $\mathrm{H}$ & 2.00053 & -3.75163 & 2.68953 \\
\hline $\mathrm{H}$ & -3.33581 & -1.59402 & 4.08782 \\
\hline $\mathrm{H}$ & -2.75235 & -3.24141 & 0.96929 \\
\hline $\mathrm{H}$ & -2.60134 & -1.48299 & 0.85606 \\
\hline $\mathrm{H}$ & -3.84985 & -2.18368 & 1.90235 \\
\hline $\mathrm{C}$ & -4.48683 & 0.98317 & 4.74157 \\
\hline $\mathrm{C}$ & 3.86277 & 1.02612 & -5.32962 \\
\hline $\mathrm{C}$ & 0.41487 & -1.08151 & -6.59655 \\
\hline $\mathrm{C}$ & -1.90311 & -1.33701 & 6.40329 \\
\hline $\mathrm{H}$ & -5.10194 & 0.18683 & 4.29866 \\
\hline $\mathrm{H}$ & -5.16951 & 1.80348 & 5.01674 \\
\hline $\mathrm{H}$ & -4.04390 & 0.60164 & 5.67104 \\
\hline $\mathrm{H}$ & 4.09724 & -0.02569 & -5.09832 \\
\hline $\mathrm{H}$ & 4.81453 & 1.54211 & -5.51717 \\
\hline $\mathrm{H}$ & 3.27658 & 1.03015 & -6.25925 \\
\hline $\mathrm{H}$ & 1.42805 & -0.90484 & -6.98273 \\
\hline $\mathrm{H}$ & -0.16402 & -0.15289 & -6.71236 \\
\hline $\mathrm{H}$ & -0.05931 & -1.83687 & -7.24336 \\
\hline $\mathrm{H}$ & -1.32604 & -1.78909 & 7.22176 \\
\hline $\mathrm{H}$ & -2.96588 & -1.56723 & 6.56124 \\
\hline $\mathrm{H}$ & -1.78722 & -0.24410 & 6.48584 \\
\hline
\end{tabular}




\subsubsection{0 $[\mathrm{P}(\mu-\mathrm{NTTer})]_{2 \mathrm{dist}}(\mathrm{CS})$}

\begin{tabular}{|c|c|c|c|}
\hline \multicolumn{4}{|c|}{102} \\
\hline $\mathrm{P}$ & 1.34572 & 0.10988 & -0.39990 \\
\hline$P$ & -1.17094 & -0.03871 & -0.96908 \\
\hline $\mathrm{N}$ & -0.09373 & 1.15755 & -0.25717 \\
\hline $\mathrm{N}$ & 0.07469 & -1.10081 & -0.31096 \\
\hline C & -0.16179 & 2.55611 & -0.19713 \\
\hline C & 0.13694 & -2.50089 & -0.28134 \\
\hline C & 1.00405 & 3.30765 & 0.12100 \\
\hline C & -1.38793 & 3.26268 & -0.37786 \\
\hline C & -1.04257 & -3.24316 & 0.00958 \\
\hline C & 1.35477 & -3.20662 & -0.48093 \\
\hline C & 0.94471 & 4.70537 & 0.16525 \\
\hline C & 2.30573 & 2.69732 & 0.51953 \\
\hline C & -1.39258 & 4.66264 & -0.31250 \\
\hline C & -2.70717 & 2.59924 & -0.57152 \\
\hline C & -1.00228 & -4.64268 & -0.02911 \\
\hline C & -2.32765 & -2.64411 & 0.47712 \\
\hline C & 1.34428 & -4.60850 & -0.48039 \\
\hline C & 2.69381 & -2.56126 & -0.62840 \\
\hline $\mathrm{H}$ & 1.85993 & 5.24509 & 0.41691 \\
\hline C & -0.23959 & 5.39791 & -0.06263 \\
\hline C & 2.44551 & 2.16170 & 1.82189 \\
\hline C & 3.43123 & 2.78511 & -0.32997 \\
\hline $\mathrm{H}$ & -2.34858 & 5.17253 & -0.44330 \\
\hline C & -3.45691 & 2.80791 & -1.75259 \\
\hline C & -3.28670 & 1.87040 & 0.49744 \\
\hline $\mathrm{H}$ & -1.92220 & -5.18574 & 0.19527 \\
\hline C & 0.17571 & -5.33678 & -0.28665 \\
\hline C & -2.40230 & -2.09561 & 1.78028 \\
\hline C & -3.50705 & -2.79965 & -0.28056 \\
\hline $\mathrm{H}$ & 2.29560 & -5.12327 & -0.62946 \\
\hline C & 3.35655 & -2.57052 & -1.87873 \\
\hline C & 3.37527 & -2.09909 & 0.52124 \\
\hline $\mathrm{H}$ & -0.27027 & 6.48668 & -0.01254 \\
\hline C & 3.70159 & 1.72549 & 2.24675 \\
\hline C & 1.26229 & 2.04964 & 2.74301 \\
\hline C & 4.66889 & 2.32845 & 0.13750 \\
\hline C & 3.31525 & 3.34936 & -1.72023 \\
\hline C & -4.70107 & 2.17906 & -1.88887 \\
\hline C & -3.00781 & 3.73430 & -2.85485 \\
\hline C & -4.54959 & 1.30305 & 0.33255 \\
\hline C & -2.57824 & 1.72395 & 1.81558 \\
\hline $\mathrm{H}$ & 0.18959 & -6.42714 & -0.29718 \\
\hline C & -3.65030 & -1.74456 & 2.29967 \\
\hline C & -1.17122 & -1.92501 & 2.62739 \\
\hline C & -4.73503 & -2.42735 & 0.27869 \\
\hline C & -3.48503 & -3.37194 & -1.67433 \\
\hline C & 4.65191 & -2.05531 & -1.96220 \\
\hline C & 2.69126 & -3.10117 & -3.11732 \\
\hline C & 4.67965 & -1.61181 & 0.39200 \\
\hline C & 2.72276 & -2.12187 & 1.87598 \\
\hline $\mathrm{H}$ & 3.80376 & 1.31729 & 3.25629 \\
\hline C & 4.83069 & 1.81233 & 1.42575 \\
\hline $\mathrm{H}$ & 0.54631 & 1.29561 & 2.37770 \\
\hline $\mathrm{H}$ & 0.70956 & 2.99805 & 2.80782 \\
\hline $\mathrm{H}$ & 1.57769 & 1.75608 & 3.75252 \\
\hline
\end{tabular}




\begin{tabular}{|c|c|c|c|}
\hline $\mathrm{H}$ & 5.53671 & 2.39494 & -0.52462 \\
\hline $\mathrm{H}$ & 4.26405 & 3.24253 & -2.26225 \\
\hline $\mathrm{H}$ & 3.04761 & 4.41629 & -1.70409 \\
\hline $\mathrm{H}$ & 2.52987 & 2.83099 & -2.29107 \\
\hline $\mathrm{H}$ & -5.26002 & 2.31859 & -2.81889 \\
\hline $\mathrm{C}$ & -5.26084 & 1.41199 & -0.86648 \\
\hline $\mathrm{H}$ & -1.92744 & 3.91229 & -2.84735 \\
\hline $\mathrm{H}$ & -3.49514 & 4.71695 & -2.74746 \\
\hline $\mathrm{H}$ & -3.29434 & 3.34008 & -3.83991 \\
\hline $\mathrm{H}$ & -4.98521 & 0.74902 & 1.16479 \\
\hline $\mathrm{H}$ & -1.76970 & 0.97918 & 1.75344 \\
\hline $\mathrm{H}$ & -3.27549 & 1.38946 & 2.59389 \\
\hline $\mathrm{H}$ & -2.11689 & 2.67015 & 2.13187 \\
\hline $\mathrm{H}$ & -3.69966 & -1.33753 & 3.31369 \\
\hline $\mathrm{C}$ & -4.83377 & -1.91789 & 1.57369 \\
\hline $\mathrm{H}$ & -0.58652 & -2.85543 & 2.67738 \\
\hline $\mathrm{H}$ & -1.43803 & -1.62468 & 3.64882 \\
\hline $\mathrm{H}$ & -0.50289 & -1.15831 & 2.20621 \\
\hline $\mathrm{H}$ & -5.64383 & -2.55246 & -0.31721 \\
\hline $\mathrm{H}$ & -2.50254 & -3.26047 & -2.14700 \\
\hline $\mathrm{H}$ & -4.23776 & -2.87845 & -2.30569 \\
\hline $\mathrm{H}$ & -3.71833 & -4.44879 & -1.67061 \\
\hline $\mathrm{H}$ & 5.15229 & -2.04965 & -2.93444 \\
\hline $\mathrm{C}$ & 5.33091 & -1.56520 & -0.84194 \\
\hline $\mathrm{H}$ & 3.40988 & -3.17089 & -3.94434 \\
\hline $\mathrm{H}$ & 1.88126 & -2.42386 & -3.42794 \\
\hline $\mathrm{H}$ & 2.24403 & -4.09137 & -2.95638 \\
\hline $\mathrm{H}$ & 5.19861 & -1.26001 & 1.28616 \\
\hline $\mathrm{H}$ & 2.17807 & -3.05983 & 2.05172 \\
\hline $\mathrm{H}$ & 1.99269 & -1.30018 & 1.97302 \\
\hline $\mathrm{H}$ & 3.46897 & -1.99001 & 2.67026 \\
\hline $\mathrm{C}$ & 6.18714 & 1.41338 & 1.94003 \\
\hline $\mathrm{C}$ & -6.58690 & 0.72469 & -1.04308 \\
\hline $\mathrm{C}$ & -6.17019 & -1.58832 & 2.18319 \\
\hline $\mathrm{C}$ & 6.71808 & -0.99837 & -0.97314 \\
\hline $\mathrm{H}$ & 6.12128 & 0.57163 & 2.64418 \\
\hline $\mathrm{H}$ & 6.65974 & 2.24956 & 2.48044 \\
\hline $\mathrm{H}$ & 6.86205 & 1.12859 & 1.12242 \\
\hline $\mathrm{H}$ & -6.44315 & -0.34947 & -1.24230 \\
\hline $\mathrm{H}$ & -7.15186 & 1.14803 & -1.88457 \\
\hline $\mathrm{H}$ & -7.20423 & 0.80444 & -0.13667 \\
\hline $\mathrm{H}$ & -6.93272 & -1.41647 & 1.41138 \\
\hline $\mathrm{H}$ & -6.11637 & -0.69266 & 2.81949 \\
\hline $\mathrm{H}$ & -6.52616 & -2.41378 & 2.82045 \\
\hline $\mathrm{H}$ & 7.27666 & -1.48748 & -1.78332 \\
\hline $\mathrm{H}$ & 7.28967 & -1.11103 & -0.04173 \\
\hline $\mathrm{H}$ & 6.67795 & 0.07789 & -1.20809 \\
\hline
\end{tabular}




\subsubsection{1 $[\mathrm{P}(\mu-\mathrm{NTer})]_{2} \cdot \mathrm{SO}$}

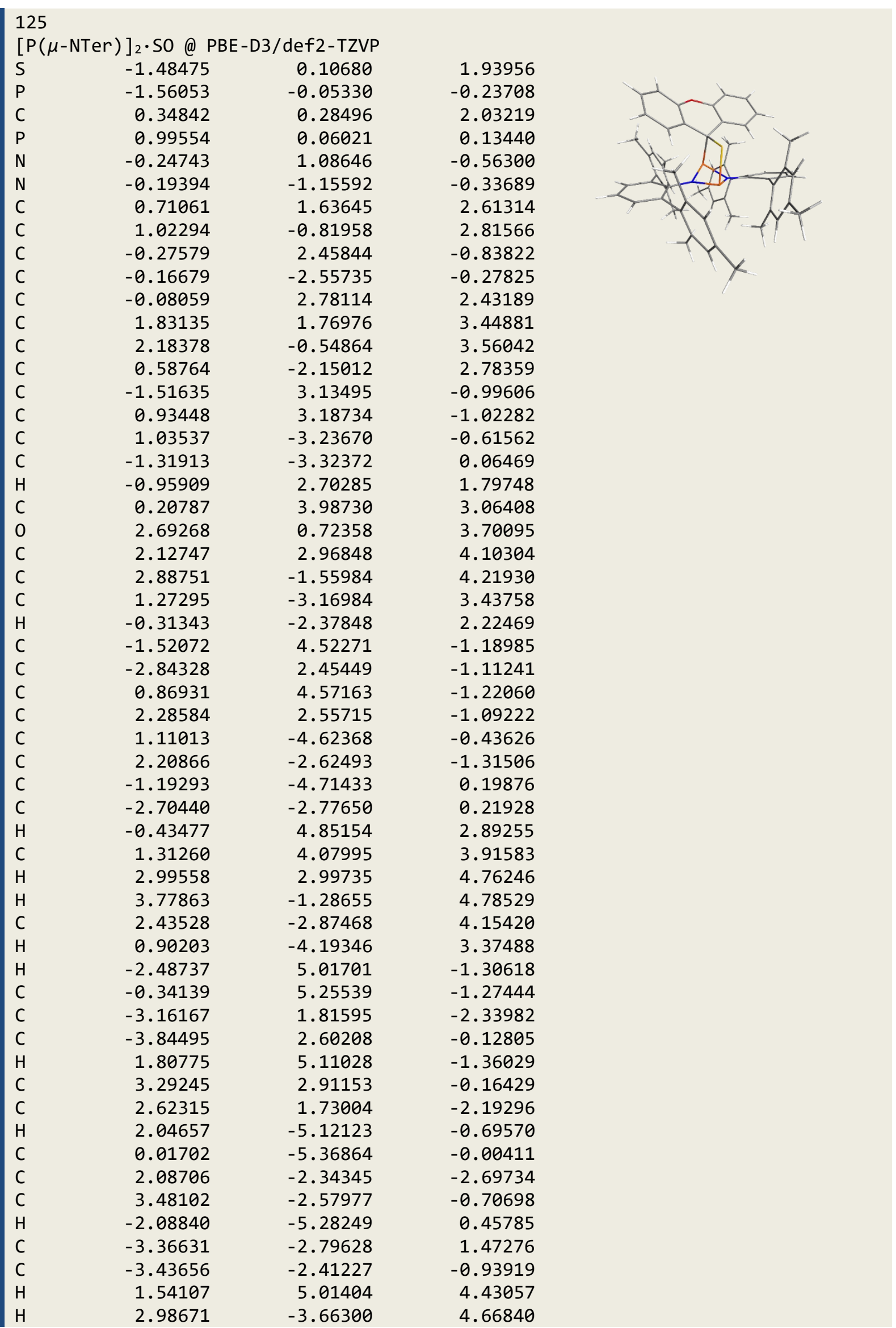




\begin{tabular}{|c|c|c|c|}
\hline $\mathrm{H}$ & -0.36571 & 6.33451 & -1.42913 \\
\hline $\mathrm{C}$ & -4.45901 & 1.35503 & -2.55666 \\
\hline $\mathrm{C}$ & -2.12611 & 1.64432 & -3.41546 \\
\hline $\mathrm{C}$ & -5.13478 & 2.11830 & -0.39069 \\
\hline $\mathrm{C}$ & -3.56972 & 3.24621 & 1.20381 \\
\hline $\mathrm{C}$ & 4.57497 & 2.37153 & -0.30485 \\
\hline $\mathrm{C}$ & 3.05441 & 3.89851 & 0.94259 \\
\hline $\mathrm{C}$ & 3.92750 & 1.24802 & -2.31277 \\
\hline $\mathrm{C}$ & 1.61749 & 1.38975 & -3.25736 \\
\hline $\mathrm{H}$ & 0.09265 & -6.44981 & 0.11706 \\
\hline $\mathrm{C}$ & 3.23628 & -2.07853 & -3.44508 \\
\hline $\mathrm{C}$ & 0.75099 & -2.38957 & -3.38518 \\
\hline $\mathrm{C}$ & 4.60561 & -2.31710 & -1.49875 \\
\hline $\mathrm{C}$ & 3.65125 & -2.79608 & 0.77018 \\
\hline $\mathrm{C}$ & -4.69661 & -2.37453 & 1.54558 \\
\hline $\mathrm{C}$ & -2.70374 & -3.23880 & 2.74939 \\
\hline $\mathrm{C}$ & -4.77046 & -2.01193 & -0.81565 \\
\hline $\mathrm{C}$ & -2.81540 & -2.46059 & -2.30702 \\
\hline $\mathrm{H}$ & -4.69539 & 0.88306 & -3.51462 \\
\hline $\mathrm{C}$ & -5.47032 & 1.51386 & -1.60195 \\
\hline $\mathrm{H}$ & -1.34933 & 0.92524 & -3.10980 \\
\hline $\mathrm{H}$ & -1.60704 & 2.58885 & -3.63259 \\
\hline $\mathrm{H}$ & -2.58256 & 1.27169 & -4.34142 \\
\hline $\mathrm{H}$ & -5.90752 & 2.25042 & 0.37190 \\
\hline $\mathrm{H}$ & -4.49456 & 3.64223 & 1.64410 \\
\hline $\mathrm{H}$ & -2.84230 & 4.06418 & 1.12523 \\
\hline $\mathrm{H}$ & -3.15558 & 2.50602 & 1.90836 \\
\hline $\mathrm{H}$ & 5.33678 & 2.63721 & 0.43359 \\
\hline $\mathrm{C}$ & 4.91793 & 1.53855 & -1.36933 \\
\hline $\mathrm{H}$ & 2.01411 & 3.90515 & 1.28014 \\
\hline $\mathrm{H}$ & 3.29044 & 4.92272 & 0.60976 \\
\hline $\mathrm{H}$ & 3.70096 & 3.68044 & 1.80180 \\
\hline $\mathrm{H}$ & 4.17341 & 0.62519 & -3.17402 \\
\hline $\mathrm{H}$ & 0.89149 & 0.64583 & -2.89578 \\
\hline $\mathrm{H}$ & 2.11484 & 0.96468 & -4.13835 \\
\hline $\mathrm{H}$ & 1.04177 & 2.27370 & -3.56612 \\
\hline $\mathrm{H}$ & 3.13098 & -1.89009 & -4.51758 \\
\hline $\mathrm{C}$ & 4.51238 & -2.09052 & -2.87246 \\
\hline $\mathrm{H}$ & 0.26453 & -3.36736 & -3.25071 \\
\hline $\mathrm{H}$ & 0.85787 & -2.19985 & -4.46098 \\
\hline $\mathrm{H}$ & 0.06394 & -1.63767 & -2.97026 \\
\hline $\mathrm{H}$ & 5.58965 & -2.31306 & -1.02189 \\
\hline $\mathrm{H}$ & 3.15289 & -3.70852 & 1.12213 \\
\hline $\mathrm{H}$ & 3.20612 & -1.96439 & 1.33807 \\
\hline $\mathrm{H}$ & 4.71415 & -2.85258 & 1.03890 \\
\hline $\mathrm{H}$ & -5.19307 & -2.38413 & 2.52000 \\
\hline $\mathrm{C}$ & -5.41736 & -1.96817 & 0.42038 \\
\hline $\mathrm{H}$ & -3.44384 & -3.68404 & 3.42818 \\
\hline $\mathrm{H}$ & -2.25607 & -2.37545 & 3.26609 \\
\hline $\mathrm{H}$ & -1.90601 & -3.97127 & 2.58049 \\
\hline $\mathrm{H}$ & -5.31805 & -1.73529 & -1.71975 \\
\hline $\mathrm{H}$ & -2.18425 & -3.34946 & -2.43898 \\
\hline $\mathrm{H}$ & -2.17373 & -1.57997 & -2.48142 \\
\hline $\mathrm{H}$ & -3.58988 & -2.45580 & -3.08495 \\
\hline $\mathrm{C}$ & -6.88201 & 1.09535 & -1.90931 \\
\hline $\mathrm{C}$ & 6.30830 & 0.98013 & -1.49372 \\
\hline $\mathrm{C}$ & 5.73768 & -1.87957 & -3.72037 \\
\hline $\mathrm{C}$ & -6.84295 & -1.50877 & 0.55112 \\
\hline $\mathrm{H}$ & -6.92659 & 0.05799 & -2.27271 \\
\hline $\mathrm{H}$ & -7.31257 & 1.73031 & -2.69949 \\
\hline
\end{tabular}




$\begin{array}{rrrr}\mathrm{H} & -7.52916 & 1.17480 & -1.02643 \\ \mathrm{H} & 6.36458 & -0.02609 & -1.04948 \\ \mathrm{H} & 7.04072 & 1.61337 & -0.97449 \\ \mathrm{H} & 6.61337 & 0.88759 & -2.54513 \\ \mathrm{H} & 6.65132 & -1.87695 & -3.11176 \\ \mathrm{H} & 5.69340 & -0.92432 & -4.26668 \\ \mathrm{H} & 5.83644 & -2.67440 & -4.47581 \\ \mathrm{H} & -7.36104 & -2.04100 & 1.36105 \\ \mathrm{H} & -7.40523 & -1.66199 & -0.37962 \\ \mathrm{H} & -6.88368 & -0.43350 & 0.78721\end{array}$




\subsubsection{2 $[\mathrm{P}(\mu-\mathrm{NTer})]_{2} \cdot \mathrm{SS}$}

\begin{tabular}{|c|c|c|c|}
\hline \multicolumn{4}{|c|}{$1, . \varsigma \varsigma \cap P R F$} \\
\hline$S$ & -1.41613 & 0.07923 & 1.97552 \\
\hline$P$ & -1.60752 & -0.01523 & -0.19310 \\
\hline C & 0.42485 & 0.20849 & 1.98590 \\
\hline$P$ & 0.96945 & 0.05047 & 0.04078 \\
\hline N & -0.29901 & 1.11624 & -0.54643 \\
\hline N & -0.26911 & -1.13592 & -0.39539 \\
\hline C & 0.84229 & 1.54936 & 2.58001 \\
\hline C & 1.07930 & -0.98854 & 2.66692 \\
\hline C & -0.33734 & 2.49295 & -0.80047 \\
\hline C & -0.26896 & -2.53889 & -0.35480 \\
\hline C & -0.03303 & 2.64680 & 2.60936 \\
\hline C & 2.10433 & 1.73443 & 3.18251 \\
\hline C & 2.39101 & -0.93208 & 3.18282 \\
\hline C & 0.42228 & -2.22227 & 2.75376 \\
\hline C & -1.58379 & 3.17065 & -0.89791 \\
\hline C & 0.86025 & 3.22705 & -1.03892 \\
\hline C & 0.90764 & -3.23576 & -0.74649 \\
\hline C & -1.42395 & -3.29115 & 0.01456 \\
\hline $\mathrm{H}$ & -1.00283 & 2.54228 & 2.13066 \\
\hline C & 0.28747 & 3.84048 & 3.25234 \\
\hline$S$ & 3.37586 & 0.52175 & 3.07061 \\
\hline C & 2.41213 & 2.91287 & 3.87779 \\
\hline C & 2.98014 & -2.05116 & 3.78950 \\
\hline C & 1.01142 & -3.34787 & 3.32309 \\
\hline $\mathrm{H}$ & -0.58166 & -2.28547 & 2.35033 \\
\hline C & -1.59958 & 4.55914 & -1.08601 \\
\hline C & -2.91866 & 2.49961 & -0.97548 \\
\hline C & 0.78335 & 4.61064 & -1.23868 \\
\hline C & 2.20923 & 2.60205 & -1.15403 \\
\hline C & 0.96921 & -4.62423 & -0.57699 \\
\hline C & 2.06481 & -2.63172 & -1.47834 \\
\hline C & -1.31310 & -4.68486 & 0.13071 \\
\hline C & -2.80053 & -2.73147 & 0.20574 \\
\hline $\mathrm{H}$ & -0.42882 & 4.66315 & 3.24107 \\
\hline C & 1.50552 & 3.96700 & 3.92149 \\
\hline $\mathrm{H}$ & 3.37758 & 2.98950 & 4.38067 \\
\hline $\mathrm{H}$ & 3.98347 & -1.95599 & 4.20812 \\
\hline C & 2.29854 & -3.26227 & 3.85561 \\
\hline $\mathrm{H}$ & 0.45960 & -4.28847 & 3.34697 \\
\hline $\mathrm{H}$ & -2.57203 & 5.05112 & -1.15326 \\
\hline C & -0.42804 & 5.29392 & -1.23207 \\
\hline C & -3.28993 & 1.90134 & -2.20859 \\
\hline C & -3.88265 & 2.63746 & 0.04578 \\
\hline $\mathrm{H}$ & 1.71291 & 5.14936 & -1.42752 \\
\hline C & 3.24162 & 2.96762 & -0.26003 \\
\hline C & 2.51336 & 1.76465 & -2.25550 \\
\hline $\mathrm{H}$ & 1.88971 & -5.13334 & -0.86799 \\
\hline C & -0.11994 & -5.35553 & -0.11339 \\
\hline C & 3.35739 & -2.64380 & -0.91952 \\
\hline C & 1.90095 & -2.29115 & -2.84467 \\
\hline $\mathrm{H}$ & -2.20914 & -5.24325 & 0.40838 \\
\hline C & -3.54062 & -2.31370 & -0.93110 \\
\hline C & -3.45740 & -2.79623 & 1.46059 \\
\hline $\mathrm{H}$ & 1.75500 & 4.88054 & 4.46282 \\
\hline $\mathrm{H}$ & 2.77064 & -4.12895 & 4.32035 \\
\hline
\end{tabular}




\begin{tabular}{|c|c|c|c|}
\hline $\mathrm{H}$ & -0.46163 & 6.37280 & -1.38659 \\
\hline $\mathrm{C}$ & -4.60391 & 1.47749 & -2.39585 \\
\hline $\mathrm{C}$ & -2.29216 & 1.73908 & -3.32088 \\
\hline $\mathrm{C}$ & -5.19226 & 2.19256 & -0.18790 \\
\hline $\mathrm{C}$ & -3.54672 & 3.23348 & 1.38618 \\
\hline $\mathrm{C}$ & 4.51391 & 2.40964 & -0.41912 \\
\hline $\mathrm{C}$ & 3.03523 & 3.98778 & 0.82319 \\
\hline $\mathrm{C}$ & 3.80849 & 1.26432 & -2.39616 \\
\hline $\mathrm{C}$ & 1.48046 & 1.43188 & -3.29600 \\
\hline $\mathrm{H}$ & -0.05702 & -6.43846 & -0.00123 \\
\hline $\mathrm{C}$ & 4.46123 & -2.36063 & -1.73485 \\
\hline $\mathrm{C}$ & 3.58389 & -2.97970 & 0.52795 \\
\hline $\mathrm{C}$ & 3.02893 & -2.01892 & -3.61932 \\
\hline $\mathrm{C}$ & 0.54268 & -2.28300 & -3.48751 \\
\hline $\mathrm{C}$ & -4.86894 & -1.90498 & -0.78153 \\
\hline $\mathrm{C}$ & -2.93712 & -2.32110 & -2.30718 \\
\hline $\mathrm{C}$ & -4.78240 & -2.36270 & 1.56056 \\
\hline $\mathrm{C}$ & -2.80972 & -3.31324 & 2.71825 \\
\hline $\mathrm{H}$ & -4.88236 & 1.03927 & -3.35856 \\
\hline $\mathrm{C}$ & -5.58130 & 1.63566 & -1.40564 \\
\hline $\mathrm{H}$ & -1.78055 & 2.68589 & -3.54672 \\
\hline $\mathrm{H}$ & -2.78034 & 1.37559 & -4.23419 \\
\hline $\mathrm{H}$ & -1.50505 & 1.01781 & -3.04888 \\
\hline $\mathrm{H}$ & -5.93708 & 2.32079 & 0.60272 \\
\hline $\mathrm{H}$ & -2.74819 & 3.98260 & 1.31524 \\
\hline $\mathrm{H}$ & -3.20056 & 2.44662 & 2.07702 \\
\hline $\mathrm{H}$ & -4.43006 & 3.70430 & 1.83848 \\
\hline $\mathrm{H}$ & 5.29516 & 2.67557 & 0.29838 \\
\hline $\mathrm{C}$ & 4.82000 & 1.54967 & -1.47373 \\
\hline $\mathrm{H}$ & 3.75529 & 3.83882 & 1.63681 \\
\hline $\mathrm{H}$ & 2.02297 & 3.95833 & 1.23961 \\
\hline $\mathrm{H}$ & 3.19121 & 5.00709 & 0.43394 \\
\hline $\mathrm{H}$ & 4.02848 & 0.62686 & -3.25339 \\
\hline $\mathrm{H}$ & 0.90774 & 2.32156 & -3.59388 \\
\hline $\mathrm{H}$ & 0.75427 & 0.69673 & -2.91717 \\
\hline $\mathrm{H}$ & 1.95324 & 0.99909 & -4.18659 \\
\hline $\mathrm{H}$ & 5.46061 & -2.38945 & -1.29108 \\
\hline $\mathrm{C}$ & 4.32506 & -2.07196 & -3.09135 \\
\hline $\mathrm{H}$ & 4.33291 & -2.30607 & 0.96791 \\
\hline $\mathrm{H}$ & 3.95849 & -4.00899 & 0.64980 \\
\hline $\mathrm{H}$ & 2.66317 & -2.90040 & 1.11453 \\
\hline $\mathrm{H}$ & 2.89146 & -1.78340 & -4.67881 \\
\hline $\mathrm{H}$ & -0.11118 & -1.52854 & -3.02654 \\
\hline $\mathrm{H}$ & 0.03713 & -3.25272 & -3.36646 \\
\hline $\mathrm{H}$ & 0.61842 & -2.06193 & -4.55997 \\
\hline $\mathrm{H}$ & -5.41998 & -1.58861 & -1.67035 \\
\hline C & -5.50732 & -1.90366 & 0.45980 \\
\hline $\mathrm{H}$ & -2.28734 & -1.44271 & -2.46058 \\
\hline $\mathrm{H}$ & -3.72145 & -2.28060 & -3.07411 \\
\hline $\mathrm{H}$ & -2.31858 & -3.21214 & -2.47758 \\
\hline $\mathrm{H}$ & -5.27248 & -2.40993 & 2.53725 \\
\hline $\mathrm{H}$ & -1.95075 & -3.96225 & 2.51706 \\
\hline $\mathrm{H}$ & -3.53813 & -3.88386 & 3.31119 \\
\hline $\mathrm{H}$ & -2.46139 & -2.47718 & 3.34471 \\
\hline C & -7.01305 & 1.26808 & -1.68392 \\
\hline C & 6.19336 & 0.95342 & -1.61024 \\
\hline C & 5.52309 & -1.83338 & -3.97031 \\
\hline C & -6.92808 & -1.43943 & 0.62057 \\
\hline $\mathrm{H}$ & -7.44671 & 1.94143 & -2.43992 \\
\hline $\mathrm{H}$ & -7.63229 & 1.33693 & -0.78036 \\
\hline
\end{tabular}




$\begin{array}{rrrr}\mathrm{H} & -7.09916 & 0.24527 & -2.07961 \\ \mathrm{H} & 6.49387 & 0.86835 & -2.66382 \\ \mathrm{H} & 6.21895 & -0.06165 & -1.18323 \\ \mathrm{H} & 6.94541 & 1.55551 & -1.08226 \\ \mathrm{H} & 5.46709 & -0.85690 & -4.47661 \\ \mathrm{H} & 5.59197 & -2.59783 & -4.75974 \\ \mathrm{H} & 6.45603 & -1.85991 & -3.39223 \\ \mathrm{H} & -6.95923 & -0.37237 & 0.89213 \\ \mathrm{H} & -7.44109 & -1.99502 & 1.41793 \\ \mathrm{H} & -7.50124 & -1.55834 & -0.30851\end{array}$

\subsubsection{3 $[\mathrm{P}(\mu-\mathrm{NTer})]_{2} \cdot \mathrm{OO}$}

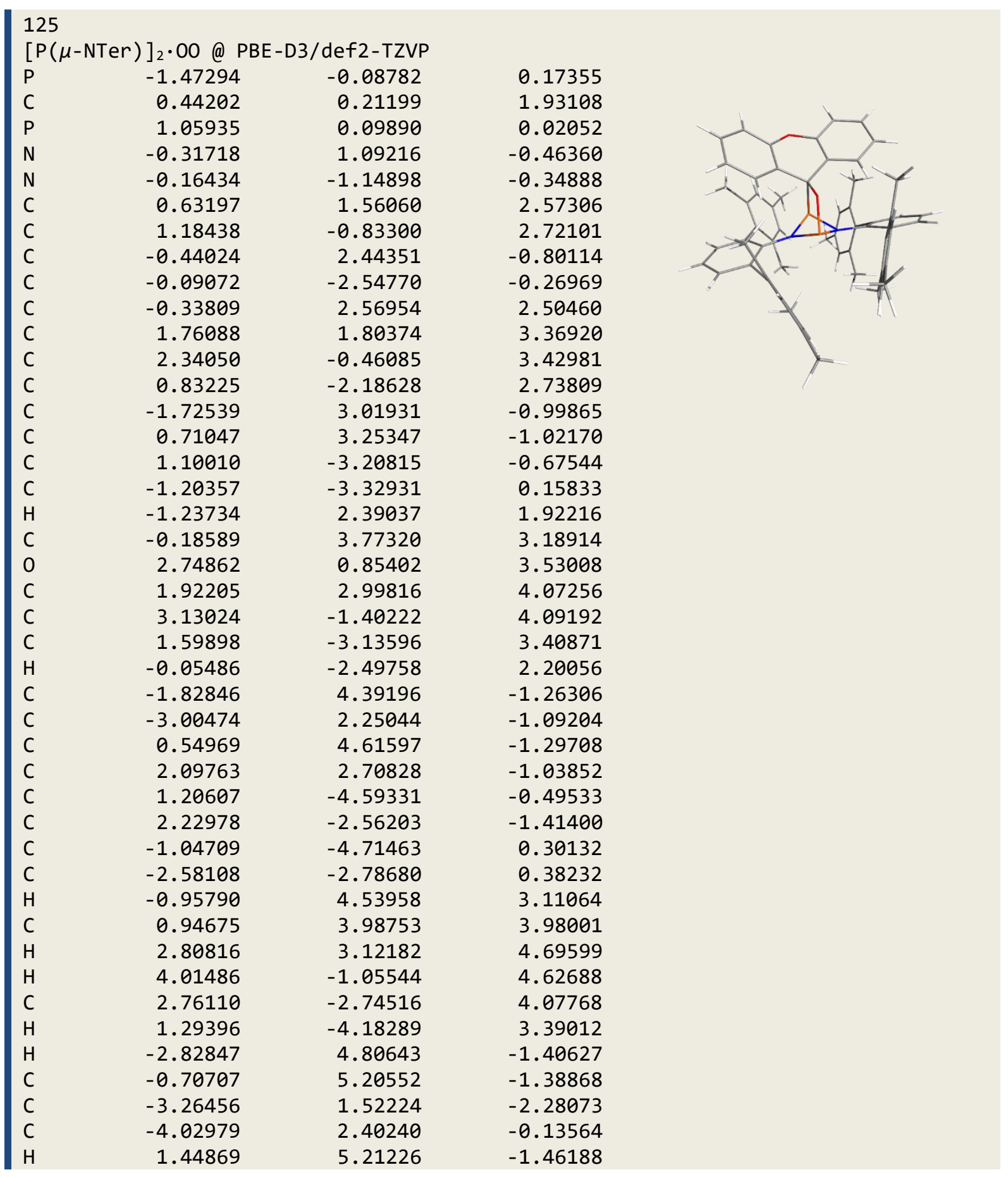




\begin{tabular}{|c|c|c|c|}
\hline C & 3.03936 & 3.10674 & -0.06179 \\
\hline C & 2.51812 & 1.88827 & -2.11311 \\
\hline $\mathrm{H}$ & 2.13284 & -5.07847 & -0.80785 \\
\hline C & 0.15717 & -5.35133 & 0.01717 \\
\hline C & 2.04627 & -2.24124 & -2.78113 \\
\hline C & 3.52278 & -2.48608 & -0.85242 \\
\hline $\mathrm{H}$ & -1.91250 & -5.29364 & 0.62978 \\
\hline C & -3.16770 & -2.73903 & 1.67025 \\
\hline C & -3.37799 & -2.47538 & -0.74916 \\
\hline $\mathrm{H}$ & 1.06908 & 4.92169 & 4.52965 \\
\hline $\mathrm{H}$ & 3.37675 & -3.48082 & 4.59700 \\
\hline $\mathrm{H}$ & -0.81126 & 6.26992 & -1.60101 \\
\hline C & -4.54027 & 1.00325 & -2.50000 \\
\hline C & -2.19667 & 1.33310 & -3.32216 \\
\hline C & -5.29841 & 1.86904 & -0.40220 \\
\hline C & -3.79652 & 3.09491 & 1.18136 \\
\hline C & 4.33718 & 2.58843 & -0.11389 \\
\hline C & 2.70241 & 4.12113 & 0.99332 \\
\hline C & 3.83677 & 1.43127 & -2.14817 \\
\hline C & 1.57513 & 1.50761 & -3.22052 \\
\hline $\mathrm{H}$ & 0.25966 & -6.42977 & 0.14255 \\
\hline C & 3.15391 & -1.89651 & -3.55901 \\
\hline C & 0.68772 & -2.31460 & -3.42055 \\
\hline C & 4.60387 & -2.14241 & -1.67325 \\
\hline C & 3.76461 & -2.73929 & 0.60915 \\
\hline C & -4.49845 & -2.32641 & 1.79663 \\
\hline C & -2.42588 & -3.09678 & 2.92991 \\
\hline C & -4.70713 & -2.08953 & -0.57226 \\
\hline C & -2.81336 & -2.54011 & -2.14027 \\
\hline $\mathrm{H}$ & -4.73465 & 0.46670 & -3.43324 \\
\hline C & -5.58430 & 1.19031 & -1.58630 \\
\hline $\mathrm{H}$ & -1.37568 & 0.70571 & -2.94172 \\
\hline H & -1.74419 & 2.29200 & -3.61424 \\
\hline $\mathrm{H}$ & -2.60539 & 0.84985 & -4.21889 \\
\hline $\mathrm{H}$ & -6.09372 & 2.01652 & 0.33426 \\
\hline $\mathrm{H}$ & -4.71504 & 3.57882 & 1.54073 \\
\hline $\mathrm{H}$ & -3.00581 & 3.85248 & 1.11724 \\
\hline $\mathrm{H}$ & -3.49503 & 2.36310 & 1.94956 \\
\hline $\mathrm{H}$ & 5.04789 & 2.87766 & 0.66541 \\
\hline C & 4.75575 & 1.74071 & -1.14175 \\
\hline $\mathrm{H}$ & 1.66482 & 4.04010 & 1.33400 \\
\hline H & 2.82946 & 5.14335 & 0.60088 \\
\hline $\mathrm{H}$ & 3.36715 & 4.01824 & 1.85946 \\
\hline $\mathrm{H}$ & 4.14700 & 0.79995 & -2.98139 \\
\hline $\mathrm{H}$ & 0.86390 & 0.73466 & -2.88958 \\
\hline $\mathrm{H}$ & 2.12678 & 1.09815 & -4.07607 \\
\hline $\mathrm{H}$ & 0.97800 & 2.36674 & -3.55684 \\
\hline H & 3.00021 & -1.67465 & -4.61915 \\
\hline C & 4.44898 & -1.86383 & -3.03143 \\
\hline $\mathrm{H}$ & 0.22661 & -3.30244 & -3.27321 \\
\hline $\mathrm{H}$ & 0.75061 & -2.11629 & -4.49824 \\
\hline $\mathrm{H}$ & 0.00176 & -1.57889 & -2.97496 \\
\hline $\mathrm{H}$ & 5.60382 & -2.10738 & -1.23257 \\
\hline $\mathrm{H}$ & 3.24965 & -3.63675 & 0.97311 \\
\hline H & 3.38804 & -1.90050 & 1.21558 \\
\hline $\mathrm{H}$ & 4.83786 & -2.84221 & 0.81666 \\
\hline $\mathrm{H}$ & -4.93801 & -2.28688 & 2.79723 \\
\hline C & -5.28722 & -1.99317 & 0.69534 \\
\hline $\mathrm{H}$ & -3.12784 & -3.45166 & 3.69653 \\
\hline H & -1.90882 & -2.21498 & 3.33769 \\
\hline
\end{tabular}




$\begin{array}{lrrr}H & -1.67202 & -3.87664 & 2.76779 \\ H & -5.30628 & -1.86086 & -1.45615 \\ H & -2.22780 & -3.45532 & -2.30338 \\ H & -2.13655 & -1.69134 & -2.33049 \\ H & -3.61531 & -2.49331 & -2.88817 \\ \mathrm{C} & -6.97549 & 0.71459 & -1.90567 \\ \mathrm{C} & 6.15116 & 1.18020 & -1.15924 \\ \mathrm{C} & 5.63088 & -1.54143 & -3.90589 \\ \mathrm{C} & -6.71120 & -1.54697 & 0.87806 \\ \mathrm{H} & -6.98384 & -0.34282 & -2.21022 \\ \mathrm{H} & -7.40264 & 1.29092 & -2.74133 \\ \mathrm{H} & -7.64783 & 0.82515 & -1.04508 \\ \mathrm{H} & 6.20027 & 0.24559 & -0.57803 \\ \mathrm{H} & 6.87146 & 1.88149 & -0.71510 \\ \mathrm{H} & 6.47825 & 0.94584 & -2.18122 \\ \mathrm{H} & 6.56769 & -1.55701 & -3.33337 \\ \mathrm{H} & 5.53400 & -0.54564 & -4.36732 \\ \mathrm{H} & 5.72487 & -2.26539 & -4.72981 \\ \mathrm{H} & -7.15685 & -1.99185 & 1.77845 \\ \mathrm{H} & -7.33357 & -1.81518 & 0.01371 \\ \mathrm{H} & -6.76156 & -0.45242 & 0.99127 \\ \mathrm{O} & -0.97324 & -0.04551 & 1.79482\end{array}$

\subsubsection{4 $[\mathrm{P}(\mu-\mathrm{NTer})]_{2} \cdot \mathrm{Fc}$}

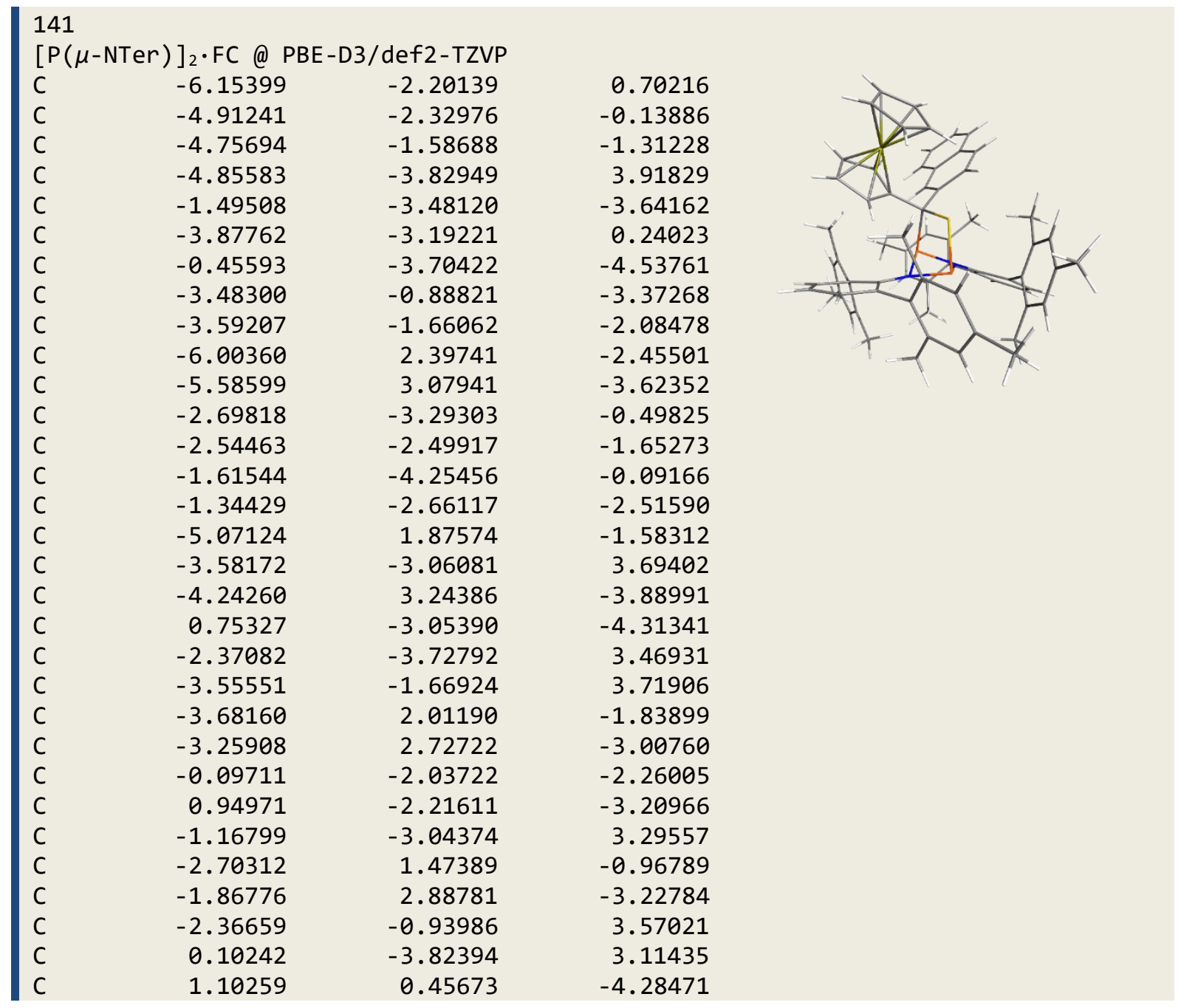




\begin{tabular}{|c|c|c|c|}
\hline C & 3.38215 & -3.53199 & -2.16927 \\
\hline C & -1.15457 & -1.62558 & 3.35477 \\
\hline C & 2.25457 & -1.49129 & -3.15794 \\
\hline C & -1.34590 & 1.67701 & -1.17696 \\
\hline C & -0.94387 & 2.39132 & -2.33395 \\
\hline C & -2.43385 & 0.55715 & 3.67013 \\
\hline C & 2.32387 & -0.18669 & -3.69419 \\
\hline C & 3.43514 & -2.13999 & -2.73172 \\
\hline C & -0.36700 & 1.24950 & -0.09608 \\
\hline C & 0.17492 & -0.93927 & 3.46655 \\
\hline C & -1.67674 & 2.98094 & 1.41862 \\
\hline C & 3.55335 & 0.47747 & -3.71067 \\
\hline C & 4.64684 & -1.44901 & -2.78941 \\
\hline C & 1.12698 & -0.80031 & 2.41461 \\
\hline C & -0.54124 & 2.13559 & 1.12154 \\
\hline C & 3.37429 & -3.09858 & 1.82803 \\
\hline C & 0.53318 & -0.54494 & 4.76119 \\
\hline C & 4.72547 & -0.13114 & -3.25282 \\
\hline c & -1.44161 & 3.64594 & 2.66303 \\
\hline C & 0.39778 & 2.32106 & 2.19699 \\
\hline C & 4.12868 & -1.85419 & 1.45999 \\
\hline C & 2.47336 & -0.46471 & 2.74309 \\
\hline C & -0.25796 & 5.40201 & -0.26216 \\
\hline C & -0.15671 & 3.23758 & 3.14380 \\
\hline C & 1.81523 & -0.09682 & 5.06739 \\
\hline C & 6.02825 & 0.62076 & -3.24454 \\
\hline C & 3.64764 & -0.56716 & 1.81673 \\
\hline C & 5.33738 & -1.97576 & 0.77243 \\
\hline C & 0.99466 & 4.71806 & -0.38283 \\
\hline C & 2.77913 & -0.11085 & 4.06581 \\
\hline C & -0.24914 & 6.11921 & 0.97934 \\
\hline c & 4.42141 & 0.57042 & 1.47683 \\
\hline C & 6.10967 & -0.86362 & 0.42868 \\
\hline C & 1.77350 & 5.00124 & 0.78448 \\
\hline C & 1.00569 & 5.86993 & 1.62707 \\
\hline C & 5.62188 & 0.39660 & 0.77729 \\
\hline C & 7.44897 & -1.02706 & -0.23610 \\
\hline C & 3.99458 & 1.96999 & 1.82265 \\
\hline $\mathrm{H}$ & -6.95583 & -1.68457 & 0.15736 \\
\hline $\mathrm{H}$ & -2.46650 & -3.95637 & -3.79351 \\
\hline $\mathrm{H}$ & -6.53161 & -3.18463 & 1.01856 \\
\hline $\mathrm{H}$ & -5.06502 & -4.51984 & 3.08614 \\
\hline $\mathrm{H}$ & -0.59057 & -4.35509 & -5.40202 \\
\hline $\mathrm{H}$ & -3.45356 & -1.56562 & -4.23945 \\
\hline $\mathrm{H}$ & -5.56839 & -0.93640 & -1.64776 \\
\hline $\mathrm{H}$ & -5.71694 & -3.15602 & 4.02091 \\
\hline $\mathrm{H}$ & -7.06913 & 2.28602 & -2.24717 \\
\hline $\mathrm{H}$ & -6.33299 & 3.48169 & -4.30965 \\
\hline $\mathrm{H}$ & -5.94540 & -1.62826 & 1.61997 \\
\hline $\mathrm{H}$ & -3.98719 & -3.80136 & 1.13798 \\
\hline $\mathrm{H}$ & -4.33498 & -0.20883 & -3.49645 \\
\hline $\mathrm{H}$ & -4.79331 & -4.44128 & 4.83160 \\
\hline $\mathrm{H}$ & -1.39902 & -4.97075 & -0.89880 \\
\hline $\mathrm{H}$ & -1.90188 & -4.81096 & 0.80859 \\
\hline $\mathrm{H}$ & -2.55886 & -0.29325 & -3.41099 \\
\hline $\mathrm{H}$ & -5.38600 & 1.34503 & -0.68253 \\
\hline $\mathrm{H}$ & -2.35732 & -4.82174 & 3.45212 \\
\hline $\mathrm{H}$ & -3.91635 & 3.77868 & -4.78483 \\
\hline $\mathrm{H}$ & 1.58075 & -3.16750 & -5.01710 \\
\hline $\mathrm{H}$ & -0.67220 & -3.72849 & 0.12169 \\
\hline
\end{tabular}




\begin{tabular}{|c|c|c|c|}
\hline $\mathrm{H}$ & -4.48605 & -1.11980 & 3.89074 \\
\hline $\mathrm{H}$ & -0.08907 & -4.90331 & 3.17051 \\
\hline $\mathrm{H}$ & -3.04450 & 0.91802 & -0.09201 \\
\hline $\mathrm{H}$ & 0.78596 & -0.07360 & -5.19691 \\
\hline $\mathrm{H}$ & 2.91329 & -4.24070 & -2.86655 \\
\hline $\mathrm{H}$ & -1.53262 & 3.42908 & -4.11573 \\
\hline $\mathrm{H}$ & 2.77669 & -3.55072 & -1.24736 \\
\hline $\mathrm{H}$ & -3.08501 & 0.97042 & 2.88530 \\
\hline $\mathrm{H}$ & 0.25105 & 0.42543 & -3.59310 \\
\hline $\mathrm{H}$ & 0.56351 & -3.60884 & 2.14056 \\
\hline $\mathrm{H}$ & 0.84576 & -3.56097 & 3.88159 \\
\hline $\mathrm{H}$ & 1.29677 & 1.50519 & -4.54610 \\
\hline $\mathrm{H}$ & 4.38764 & -3.89456 & -1.91982 \\
\hline $\mathrm{H}$ & -2.55142 & 3.11626 & 0.79241 \\
\hline $\mathrm{H}$ & 0.12033 & 2.54177 & -2.50722 \\
\hline $\mathrm{H}$ & -2.85965 & 0.86867 & 4.63659 \\
\hline $\mathrm{H}$ & -1.45215 & 1.02588 & 3.56616 \\
\hline $\mathrm{H}$ & 2.45721 & -3.19826 & 1.22486 \\
\hline $\mathrm{H}$ & 3.98570 & -3.99288 & 1.65245 \\
\hline $\mathrm{H}$ & -0.21785 & -0.63936 & 5.54769 \\
\hline $\mathrm{H}$ & 3.05973 & -3.08560 & 2.88039 \\
\hline $\mathrm{H}$ & 3.59776 & 1.49532 & -4.10781 \\
\hline $\mathrm{H}$ & 5.55436 & -1.95002 & -2.44563 \\
\hline $\mathrm{H}$ & -2.11178 & 4.35693 & 3.13928 \\
\hline $\mathrm{H}$ & -1.07827 & 5.35657 & -0.97360 \\
\hline $\mathrm{H}$ & 5.69490 & -2.97722 & 0.51741 \\
\hline $\mathrm{H}$ & 1.29557 & 4.07163 & -1.20134 \\
\hline $\mathrm{H}$ & 1.36594 & 1.84532 & 2.27314 \\
\hline $\mathrm{H}$ & 6.88699 & -0.05806 & -3.33663 \\
\hline $\mathrm{H}$ & 6.07475 & 1.35265 & -4.06291 \\
\hline $\mathrm{H}$ & -1.06383 & 6.71911 & 1.37628 \\
\hline $\mathrm{H}$ & 2.07415 & 0.19617 & 6.08537 \\
\hline $\mathrm{H}$ & 0.32899 & 3.57510 & 4.05555 \\
\hline $\mathrm{H}$ & 6.14705 & 1.17595 & -2.30002 \\
\hline $\mathrm{H}$ & 7.42798 & -1.81536 & -1.00240 \\
\hline $\mathrm{H}$ & 3.15147 & 2.27764 & 1.18457 \\
\hline $\mathrm{H}$ & 3.81628 & 0.13792 & 4.29886 \\
\hline $\mathrm{H}$ & 2.76322 & 4.61000 & 1.00152 \\
\hline $\mathrm{H}$ & 7.78043 & -0.09470 & -0.71160 \\
\hline $\mathrm{H}$ & 3.65941 & 2.06289 & 2.86403 \\
\hline $\mathrm{H}$ & 1.30911 & 6.25000 & 2.59897 \\
\hline $\mathrm{H}$ & 6.20786 & 1.28384 & 0.52141 \\
\hline $\mathrm{H}$ & 8.21652 & -1.31511 & 0.50008 \\
\hline $\mathrm{H}$ & 4.82151 & 2.67465 & 1.66238 \\
\hline $\mathrm{Fe}$ & -0.00174 & 4.11973 & 1.31227 \\
\hline $\mathrm{N}$ & 0.13741 & -1.28938 & -1.09413 \\
\hline $\mathrm{N}$ & 0.74093 & -1.04560 & 1.08899 \\
\hline $\mathrm{P}$ & -0.78299 & -0.68264 & 0.27311 \\
\hline$P$ & 1.69874 & -0.87849 & -0.39416 \\
\hline $\mathrm{S}$ & 1.40564 & 1.26074 & -0.60863 \\
\hline
\end{tabular}




\subsubsection{5 $[\mathrm{P}(\mu-\mathrm{NTer})]_{2} \cdot \mathrm{CO}$}

\begin{tabular}{|c|c|c|c|}
\hline \multicolumn{4}{|c|}{$\begin{array}{l}112 \\
{[\mathrm{P}(\mu-\mathrm{NTer})]_{2} \cdot \mathrm{CO} @ \mathrm{PBE}-\mathrm{D} 3 / \text { def2-TZVP }}\end{array}$} \\
\hline$P$ & -1.32006 & 0.10247 & 0.40134 \\
\hline C & 0.35029 & -0.01149 & 2.38601 \\
\hline$P$ & 1.20632 & -0.04716 & 0.64831 \\
\hline $\mathrm{N}$ & 0.07371 & 1.15601 & 0.02333 \\
\hline $\mathrm{N}$ & -0.08458 & -1.09460 & 0.00645 \\
\hline C & 0.14628 & 2.54962 & -0.06989 \\
\hline C & -0.14935 & -2.49448 & 0.05076 \\
\hline C & -1.01644 & 3.28654 & -0.42837 \\
\hline C & 1.36680 & 3.26361 & 0.11358 \\
\hline C & 1.01539 & -3.25066 & -0.25581 \\
\hline C & -1.35855 & -3.18298 & 0.33740 \\
\hline C & -0.96206 & 4.68292 & -0.50096 \\
\hline C & -2.30506 & 2.65162 & -0.83022 \\
\hline C & 1.36876 & 4.66173 & 0.02005 \\
\hline C & 2.67801 & 2.59551 & 0.34305 \\
\hline C & 0.97470 & -4.64707 & -0.15469 \\
\hline C & 2.27776 & -2.65590 & -0.78775 \\
\hline C & -1.35145 & -4.58272 & 0.40640 \\
\hline C & -2.68150 & -2.50822 & 0.50089 \\
\hline $\mathrm{H}$ & -1.87476 & 5.21363 & -0.78009 \\
\hline C & 0.21609 & 5.38549 & -0.26492 \\
\hline C & -2.42418 & 2.08924 & -2.12057 \\
\hline C & -3.43188 & 2.71549 & 0.01695 \\
\hline $\mathrm{H}$ & 2.31987 & 5.17899 & 0.15916 \\
\hline C & 3.38623 & 2.78083 & 1.55363 \\
\hline C & 3.28388 & 1.86722 & -0.71061 \\
\hline $\mathrm{H}$ & 1.88416 & -5.20326 & -0.39048 \\
\hline C & -0.19226 & -5.32242 & 0.19252 \\
\hline C & 2.30138 & -2.18543 & -2.12419 \\
\hline C & 3.47846 & -2.70861 & -0.04997 \\
\hline $\mathrm{H}$ & -2.29425 & -5.08649 & 0.62897 \\
\hline C & -3.29481 & -2.40126 & 1.76885 \\
\hline C & -3.39566 & -2.11005 & -0.65614 \\
\hline $\mathrm{H}$ & 0.24226 & 6.47323 & -0.33558 \\
\hline C & -3.67202 & 1.62711 & -2.54703 \\
\hline C & -1.23127 & 1.98277 & -3.03090 \\
\hline C & -4.66332 & 2.25096 & -0.45554 \\
\hline C & -3.30936 & 3.22883 & 1.42718 \\
\hline C & 4.61637 & 2.13512 & 1.72807 \\
\hline C & 2.90180 & 3.70303 & 2.64381 \\
\hline C & 4.53447 & 1.28279 & -0.50733 \\
\hline C & 2.60920 & 1.72246 & -2.04668 \\
\hline $\mathrm{H}$ & -0.20646 & -6.41084 & 0.25841 \\
\hline C & 3.51960 & -1.81197 & -2.69455 \\
\hline C & 1.04262 & -2.10563 & -2.94350 \\
\hline C & 4.67838 & -2.33317 & -0.66720 \\
\hline C & 3.51025 & -3.09859 & 1.40470 \\
\hline C & -4.58569 & -1.86844 & 1.85642 \\
\hline C & -2.59722 & -2.80716 & 3.03815 \\
\hline C & -4.68890 & -1.60416 & -0.52122 \\
\hline C & -2.77234 & -2.20298 & -2.02114 \\
\hline $\mathrm{H}$ & -3.76062 & 1.19992 & -3.55006 \\
\hline C & -4.81014 & 1.71995 & -1.74017 \\
\hline $\mathrm{H}$ & -0.49084 & 1.27317 & -2.62910 \\
\hline $\mathrm{H}$ & -0.71500 & 2.94855 & -3.13328 \\
\hline
\end{tabular}




\begin{tabular}{|c|c|c|c|}
\hline $\mathrm{H}$ & -1.52919 & 1.63722 & -4.02932 \\
\hline $\mathrm{H}$ & -5.53519 & 2.31071 & 0.20187 \\
\hline $\mathrm{H}$ & -4.28396 & 3.21765 & 1.93241 \\
\hline $\mathrm{H}$ & -2.91152 & 4.25300 & 1.46086 \\
\hline $\mathrm{H}$ & -2.61714 & 2.60118 & 2.01154 \\
\hline $\mathrm{H}$ & 5.14335 & 2.25810 & 2.67891 \\
\hline C & 5.20308 & 1.37118 & 0.71676 \\
\hline $\mathrm{H}$ & 1.81767 & 3.85566 & 2.61875 \\
\hline $\mathrm{H}$ & 3.36691 & 4.69640 & 2.53640 \\
\hline $\mathrm{H}$ & 3.18370 & 3.32221 & 3.63544 \\
\hline $\mathrm{H}$ & 4.99067 & 0.72586 & -1.32671 \\
\hline $\mathrm{H}$ & 1.80332 & 0.97219 & -2.00553 \\
\hline $\mathrm{H}$ & 3.32568 & 1.38962 & -2.80818 \\
\hline $\mathrm{H}$ & 2.15097 & 2.66666 & -2.37259 \\
\hline $\mathrm{H}$ & 3.52790 & -1.46326 & -3.73126 \\
\hline C & 4.72614 & -1.89616 & -1.99018 \\
\hline $\mathrm{H}$ & 0.48793 & -3.05517 & -2.92352 \\
\hline $\mathrm{H}$ & 1.27073 & -1.85584 & -3.98771 \\
\hline $\mathrm{H}$ & 0.36341 & -1.33669 & -2.54424 \\
\hline $\mathrm{H}$ & 5.60545 & -2.38585 & -0.08925 \\
\hline $\mathrm{H}$ & 2.59577 & -3.61320 & 1.72029 \\
\hline $\mathrm{H}$ & 3.61859 & -2.19614 & 2.02925 \\
\hline $\mathrm{H}$ & 4.36917 & -3.74881 & 1.62406 \\
\hline $\mathrm{H}$ & -5.05133 & -1.78364 & 2.84251 \\
\hline C & -5.30125 & -1.46512 & 0.72831 \\
\hline $\mathrm{H}$ & -3.31374 & -3.22258 & 3.76033 \\
\hline $\mathrm{H}$ & -2.12847 & -1.92570 & 3.50045 \\
\hline $\mathrm{H}$ & -1.80813 & -3.54768 & 2.86236 \\
\hline $\mathrm{H}$ & -5.23424 & -1.31067 & -1.42070 \\
\hline $\mathrm{H}$ & -2.27837 & -3.17148 & -2.18211 \\
\hline $\mathrm{H}$ & -2.00183 & -1.42497 & -2.15183 \\
\hline $\mathrm{H}$ & -3.52572 & -2.05361 & -2.80533 \\
\hline C & -6.15869 & 1.30316 & -2.26180 \\
\hline C & 6.51007 & 0.65991 & 0.93746 \\
\hline C & 6.03180 & -1.54015 & -2.64949 \\
\hline C & -6.68419 & -0.88811 & 0.85948 \\
\hline $\mathrm{H}$ & -6.07498 & 0.47614 & -2.98133 \\
\hline $\mathrm{H}$ & -6.64977 & 2.13904 & -2.78603 \\
\hline $\mathrm{H}$ & -6.82792 & 0.98894 & -1.44997 \\
\hline $\mathrm{H}$ & 6.33755 & -0.37247 & 1.28272 \\
\hline $\mathrm{H}$ & 7.12155 & 1.16385 & 1.69863 \\
\hline $\mathrm{H}$ & 7.09414 & 0.59718 & 0.00860 \\
\hline $\mathrm{H}$ & 6.82742 & -1.38848 & -1.90730 \\
\hline $\mathrm{H}$ & 5.94412 & -0.62341 & -3.25180 \\
\hline $\mathrm{H}$ & 6.36236 & -2.34025 & -3.33092 \\
\hline $\mathrm{H}$ & -7.20005 & -1.28016 & 1.74687 \\
\hline $\mathrm{H}$ & -7.29913 & -1.10935 & -0.02389 \\
\hline $\mathrm{H}$ & -6.64044 & 0.20819 & 0.96396 \\
\hline 0 & -1.07551 & 0.07467 & 2.06431 \\
\hline C & 0.64049 & -1.29544 & 3.14857 \\
\hline $\mathrm{H}$ & 1.71340 & -1.36000 & 3.37979 \\
\hline $\mathrm{H}$ & 0.08855 & -1.30252 & 4.10088 \\
\hline $\mathrm{H}$ & 0.35454 & -2.18763 & 2.57809 \\
\hline C & 0.72008 & 1.21956 & 3.19095 \\
\hline $\mathrm{H}$ & 0.17491 & 1.21251 & 4.14786 \\
\hline $\mathrm{H}$ & 1.79717 & 1.23812 & 3.40463 \\
\hline $\mathrm{H}$ & 0.45125 & 2.13572 & 2.65185 \\
\hline
\end{tabular}




\subsubsection{6 $[\mathrm{P}(\mu-\mathrm{NTer})]_{2} \cdot \mathrm{CS}$}

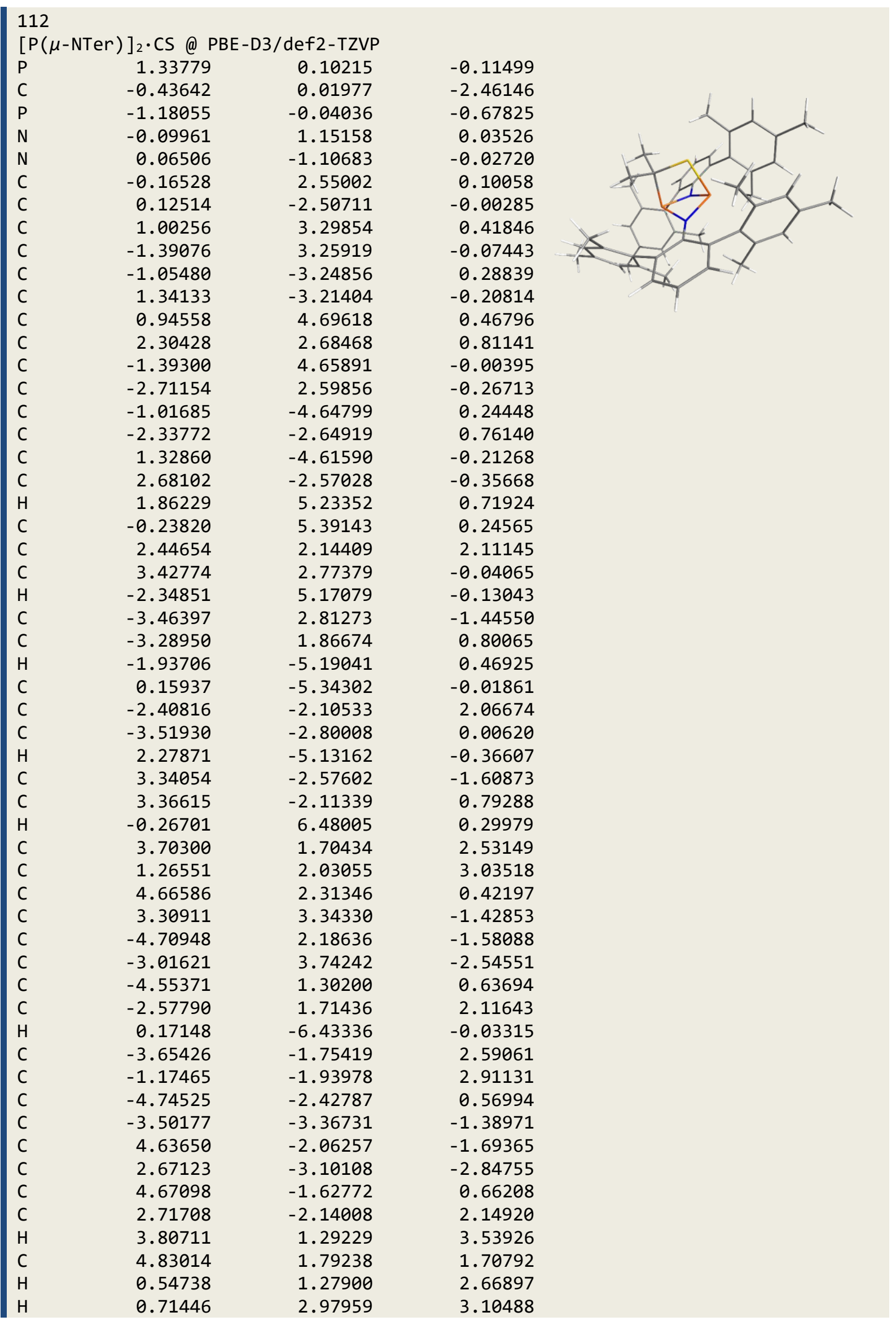




\begin{tabular}{|c|c|c|c|}
\hline $\mathrm{H}$ & 1.58302 & 1.73280 & 4.04281 \\
\hline $\mathrm{H}$ & 5.53209 & 2.38099 & -0.24212 \\
\hline $\mathrm{H}$ & 4.25635 & 3.23694 & -1.97338 \\
\hline $\mathrm{H}$ & 3.04321 & 4.41059 & -1.40782 \\
\hline $\mathrm{H}$ & 2.52144 & 2.82827 & -1.99925 \\
\hline $\mathrm{H}$ & -5.27059 & 2.33017 & -2.50895 \\
\hline C & -5.26785 & 1.41646 & -0.55986 \\
\hline $\mathrm{H}$ & -1.93555 & 3.91868 & -2.54014 \\
\hline $\mathrm{H}$ & -3.50170 & 4.72546 & -2.43329 \\
\hline $\mathrm{H}$ & -3.30590 & 3.35226 & -3.53127 \\
\hline $\mathrm{H}$ & -4.98809 & 0.74563 & 1.46827 \\
\hline $\mathrm{H}$ & -1.77072 & 0.96854 & 2.04950 \\
\hline $\mathrm{H}$ & -3.27369 & 1.37813 & 2.89530 \\
\hline $\mathrm{H}$ & -2.11423 & 2.65866 & 2.43499 \\
\hline $\mathrm{H}$ & -3.70037 & -1.35080 & 3.60623 \\
\hline C & -4.83986 & -1.92299 & 1.86704 \\
\hline $\mathrm{H}$ & -0.59131 & -2.87130 & 2.95641 \\
\hline $\mathrm{H}$ & -1.43836 & -1.64276 & 3.93451 \\
\hline $\mathrm{H}$ & -0.50616 & -1.17261 & 2.49122 \\
\hline $\mathrm{H}$ & -5.65578 & -2.54936 & -0.02408 \\
\hline $\mathrm{H}$ & -2.52031 & -3.25568 & -1.86449 \\
\hline $\mathrm{H}$ & -4.25532 & -2.87032 & -2.01733 \\
\hline $\mathrm{H}$ & -3.73678 & -4.44380 & -1.38932 \\
\hline $\mathrm{H}$ & 5.13440 & -2.05415 & -2.66714 \\
\hline C & 5.31915 & -1.57764 & -0.57335 \\
\hline $\mathrm{H}$ & 3.38762 & -3.16893 & -3.67665 \\
\hline $\mathrm{H}$ & 1.86153 & -2.42136 & -3.15361 \\
\hline $\mathrm{H}$ & 2.22283 & -4.09116 & -2.68907 \\
\hline $\mathrm{H}$ & 5.19278 & -1.28001 & 1.55618 \\
\hline $\mathrm{H}$ & 2.17135 & -3.07781 & 2.32292 \\
\hline $\mathrm{H}$ & 1.98857 & -1.31760 & 2.25111 \\
\hline $\mathrm{H}$ & 3.46553 & -2.01231 & 2.94204 \\
\hline C & 6.18726 & 1.38940 & 2.21726 \\
\hline C & -6.59545 & 0.73191 & -0.73556 \\
\hline C & -6.17419 & -1.59352 & 2.48117 \\
\hline C & 6.70689 & -1.01253 & -0.70605 \\
\hline $\mathrm{H}$ & 6.12186 & 0.54519 & 2.91850 \\
\hline $\mathrm{H}$ & 6.66258 & 2.22285 & 2.75950 \\
\hline $\mathrm{H}$ & 6.85962 & 1.10653 & 1.39688 \\
\hline $\mathrm{H}$ & -6.45393 & -0.34174 & -0.93907 \\
\hline $\mathrm{H}$ & -7.16189 & 1.15922 & -1.57405 \\
\hline $\mathrm{H}$ & -7.21034 & 0.80933 & 0.17271 \\
\hline $\mathrm{H}$ & -6.93841 & -1.41765 & 1.71195 \\
\hline $\mathrm{H}$ & -6.11731 & -0.70029 & 3.12059 \\
\hline $\mathrm{H}$ & -6.52985 & -2.42074 & 3.11633 \\
\hline $\mathrm{H}$ & 7.26261 & -1.49957 & -1.51944 \\
\hline $\mathrm{H}$ & 7.28068 & -1.12950 & 0.22348 \\
\hline $\mathrm{H}$ & 6.66787 & 0.06464 & -0.93696 \\
\hline C & -0.78796 & -1.29782 & -3.15627 \\
\hline $\mathrm{H}$ & -1.88179 & -1.40105 & -3.23820 \\
\hline $\mathrm{H}$ & -0.37532 & -1.31626 & -4.17638 \\
\hline $\mathrm{H}$ & -0.39538 & -2.16831 & -2.61513 \\
\hline C & -1.00999 & 1.20786 & -3.22753 \\
\hline $\mathrm{H}$ & -0.63888 & 1.21036 & -4.26391 \\
\hline $\mathrm{H}$ & -2.10858 & 1.15154 & -3.25935 \\
\hline $\mathrm{H}$ & -0.71837 & 2.15591 & -2.76203 \\
\hline S & 1.38926 & 0.21762 & -2.28850 \\
\hline
\end{tabular}




\section{References}

[1] J. Bresien, T. Kröger-Badge, S. Lochbrunner, D. Michalik, H. Müller, A. Schulz, E. Zander, Chem. Sci. 2019, 10, 3486-3493.

[2] S. Scheibye, R. Shabana, S.-O. Lawesson, C. Rømming, Tetrahedron 1982, 38, 9931001.

[3] G. Mlostoń, M. Kowalczyk, A. U. Augustin, P. G. Jones, D. B. Werz, Beilstein J. Org. Chem. 2020, 16, 1288-1295.

[4] G. M. Sheldrick, Acta Crystallogr. Sect. A Found. Adv. 2015, 71, 3-8.

[5] G. M. Sheldrick, Acta Crystallogr. Sect. C Struct. Chem. 2015, 71, 3-8.

[6] G. M. Sheldrick, 2004.

[7] A. Hinz, A. Schulz, A. Villinger, Angew. Chemie - Int. Ed. 2016, 55, 12214-12218.

[8] M. J. Frisch, G. W. Trucks, H. B. Schlegel, G. E. Scuseria, M. A. Robb, J. R. Cheeseman, G. Scalmani, V. Barone, B. Mennucci, G. A. Peterson, et al., 2013.

[9] F. Neese, Wiley Interdiscip. Rev. Comput. Mol. Sci. 2018, 8, 1327.

[10] J. P. Perdew, K. Burke, M. Ernzerhof, Phys. Rev. Lett. 1996, 77, 3865-3868.

[11] J. P. Perdew, K. Burke, M. Ernzerhof, Phys. Rev. Lett. 1997, 78, 1396-1396.

[12] S. Grimme, J. Antony, S. Ehrlich, H. Krieg, J. Chem. Phys. 2010, 132, 154104.

[13] S. Grimme, S. Ehrlich, L. Goerigk, J. Comput. Chem. 2011, 32, 1456-1465.

[14] F. Weigend, R. Ahlrichs, Phys. Chem. Chem. Phys. 2005, 7, 3297-305.

[15] F. Weigend, Phys. Chem. Chem. Phys. 2006, 8, 1057.

[16] F. London, J. Phys. le Radium 1937, 8, 397-409.

[17] R. McWeeny, Phys. Rev. 1962, 126, 1028-1034.

[18] R. Ditchfield, Mol. Phys. 1974, 27, 789-807.

[19] K. Wolinski, J. F. Hinton, P. Pulay, J. Am. Chem. Soc. 1990, 112, 8251-8260.

[20] J. R. Cheeseman, G. W. Trucks, T. A. Keith, M. J. Frisch, J. Chem. Phys. 1996, 104, 5497-5509.

[21] C. Adamo, V. Barone, J. Chem. Phys. 1999, 110, 6158-6170.

[22] C. J. Jameson, A. De Dios, A. Keith Jameson, Chem. Phys. Lett. 1990, 167, 575582.

[23] C. van Wüllen, Phys. Chem. Chem. Phys. 2000, 2, 2137-2144.

[24] C. Riplinger, F. Neese, J. Chem. Phys. 2013, 138, 34106.

[25] D. G. Liakos, M. Sparta, M. K. Kesharwani, J. M. L. Martin, F. Neese, J. Chem. Theory Comput. 2015, 11, 1525-1539.

[26] C. Riplinger, P. Pinski, U. Becker, E. F. Valeev, F. Neese, J. Chem. Phys. 2016, 144, 
24109.

[27] D. G. Liakos, Y. Guo, F. Neese, J. Phys. Chem. A 2020, 124, 90-100.

[28] A. Hellweg, C. Hättig, S. Höfener, W. Klopper, Theor. Chem. Acc. 2007, 117, 587597.

[29] C. J. Cramer, Essentials of Computational Chemistry: Theories and Models, John Wiley \& Sons, Ltd, Chichester, UK, 2004.

[30] A. V. Marenich, C. J. Cramer, D. G. Truhlar, J. Phys. Chem. B 2009, 113, 6378-6396. 